\title{
Clusters of galaxies: observational properties of the diffuse radio emission
}

\author{
Luigina Feretti • Gabriele Giovannini • \\ Federica Govoni • Matteo Murgia
}

Received: 7 January 2012 / Published online: 19 May 2012

(C) The Author(s) 2012. This article is published with open access at Springerlink.com

\begin{abstract}
Clusters of galaxies, as the largest virialized systems in the Universe, are ideal laboratories to study the formation and evolution of cosmic structures. The luminous matter of clusters consists of galaxies and of an embedding intracluster medium (ICM), which has been heated to temperatures of tens of millions degrees, and thus is detected through its thermal emission in the soft X-ray regime. Most of the detailed knowledge of galaxy clusters has been obtained in recent years from the study of ICM through X-ray Astronomy. At the same time, radio observations have proved that the ICM is mixed with non-thermal components, i.e. highly relativistic particles and large-scale magnetic fields, detected through their synchrotron emission.

The knowledge of the properties of these non-thermal ICM components has increased significantly, owing to sensitive radio images and to the development of theoretical models. Diffuse synchrotron radio emission in the central and peripheral cluster regions has been found in many clusters. Moreover large-scale magnetic fields appear to be present in all galaxy clusters, as derived from Rotation Measure (RM) studies. Non-thermal components are linked to the cluster X-ray properties, and to the cluster evolutionary stage, and are crucial for a comprehensive physical description of the intracluster medium. They play an important role in the cluster formation and evolution.

We review here the observational properties of diffuse non-thermal sources detected in galaxy clusters: halos, relics and mini-halos. We discuss their classification
\end{abstract}

\footnotetext{
L. Feretti $(\bowtie)$

INAF Istituto di Radioastronomia, via Gobetti 101, 40129 Bologna, Italy

e-mail: lferetti@ira.inaf.it

G. Giovannini

Dipartimento di Astronomia, via Ranzani 1, 40127 Bologna, Italy

F. Govoni · M. Murgia

INAF Osservatorio Astronomico di Cagliari, Strada 54, Loc. Poggio dei Pini, 09012 Capoterra (Ca), Italy
} 
and properties. We report published results up to date and obtain and discuss statistical properties. We present the properties of large-scale magnetic fields in clusters and in even larger structures: filaments connecting galaxy clusters. We summarize the current models of the origin of these cluster components, and outline the improvements that are expected in this area from future developments thanks to the new generation of radio telescopes.

Keywords Galaxy groups, clusters, and superclusters - Large scale structure of the Universe · Galaxy clusters · Radio sources · Intracluster matter · Cooling flows · Halos $\cdot$ Relics $\cdot$ Mini-halos $\cdot$ Large-scale magnetic fields

\section{Introduction}

Clusters of galaxies are the largest gravitationally bound systems in the Universe, with typical masses of about $10^{15} \mathrm{M}_{\text {sun }}$, and volumes of about $100 \mathrm{Mpc}^{3}$. Most of the gravitating matter in any cluster is in the form of dark matter $(\sim 80 \%)$. Some of the luminous matter is in galaxies $(\sim 3-5 \%)$, the rest is in diffuse hot gas $(\sim 15-$ $17 \%)$, detected in X-ray through its thermal bremsstrahlung emission. This thermal plasma, consisting of particles of energies of several $\mathrm{keV}$, is commonly referred to as Intracluster Medium (ICM). Most of the detailed knowledge of galaxy clusters has been obtained in recent years from the study of ICM through X-ray Astronomy.

Clusters are formed by hierarchical structure formation processes. In this scenario, smaller units (galaxies, groups and small clusters) formed first and merged under gravitational pull to larger and larger units in the course of time. Cluster mergers are the mechanism by which clusters are assembled. Denser regions form a filamentary structure in the Universe, and clusters are formed within filaments, often at their intersection, by a combination of large and small mergers. Major cluster mergers are among the most energetic events in the Universe since the Big Bang (Sarazin 2002). During mergers, shocks are driven into the ICM, with the subsequent injection of turbulence. The merger activity, which has characterized much of the history of the Universe, appears to be continuing at the present time and explains the relative abundance of substructure and temperature gradients detected in clusters of galaxies by optical and X-ray observations.

Eventually, clusters reach a relaxed state, characterized by a giant galaxy at the center, and enhanced X-ray surface brightness peak in the core. The hot gas in the center has a high density, which implies short radiative cooling times, typically one or two order of magnitudes smaller than the Hubble time. Therefore energy losses due to X-ray emission are dramatic and produce a temperature drop towards the center. It was formerly suggested that in these conditions, the ICM plasma in the cluster core should cool and condense, giving rise to a steady, pressure-driven inward flow caused by the compression of the hot surrounding gas. Relaxed clusters were then classified as "cooling flow" clusters (Fabian 1994), with predicted gas mass deposition rates in the cluster center up to $1000 \mathrm{M}_{\text {sun }}$ year $^{-1}$. This model was the subject of much debate, when XMM-Newton spectral results failed to confirm the lines and features expected as a product of a steady state cooling flow (Peterson et al. 2001; Peterson 
and Fabian 2006). X-ray and optical studies showed that the gas cooling rates were overestimated by an order of magnitude or more (McNamara et al. 2006). The classical "cooling-flow" model has finally been replaced by the paradigm of "cool-core". Observationally cool-cores are characterized by a strong peak in the surface brightness, a significant drop in the temperature and a peak in the metal distribution (e.g. De Grandi and Molendi 2001). The cooling time is often much shorter than 1 Gyr, therefore some source of heating is necessary to balance the radiation losses. At present, it is widely accepted that the source of heating in cool-core clusters is the AGN activity of the brightest cluster galaxy at the center (see McNamara and Nulsen 2007; Böhringer and Werner 2010 for recent reviews).

Galaxy clusters are also characterized by emission in the radio band. Obvious radio sources are the individual galaxies, whose emission has been observed in recent decades with sensitive radio telescopes. It often extends well beyond the galaxy optical boundaries, out to hundreds of kiloparsec, and hence it is expected that the radio emitting regions interact with the ICM. This interaction is indeed observed in tailed radio galaxies, and radio sources filling X-ray cavities at the center of cool-core clusters (McNamara and Nulsen 2007; Feretti and Giovannini 2008).

More puzzling are diffuse extended radio sources, which cannot be obviously ascribed to individual galaxies, but are instead associated with the ICM. This radio emission represents a striking feature of clusters, since it demonstrates that the thermal ICM plasma is mixed with non-thermal components. Such components are largescale magnetic fields with a population of relativistic electrons in the cluster volume. Further demonstration of the existence of magnetic fields in the ICM is obtained by studies of the Faraday rotation of polarized radio galaxies, lying in the background or embedded within the magnetized intracluster medium.

The number density of relativistic particles is of the order of $10^{-10} \mathrm{~cm}^{-3}$, while their Lorentz factors are $\gamma \gg 1000$. Magnetic field intensities are around $\sim 0.1-1 \mu \mathrm{G}$, and the energy density of the relativistic plasma is globally $\lesssim 1 \%$ than that of the thermal gas. Non-thermal components are nevertheless important for a comprehensive physical description of the intracluster medium in galaxy clusters, and play a major role in the evolution of large-scale structures in the Universe. They can have dynamic and thermodynamic effects: magnetic fields affect the heat conduction in the ICM and the gas dynamics, relativistic particles are sources of additional pressure and undergo acceleration processes that can modify the details of the ICM heating process. Realistic cosmological simulations of galaxy cluster formation also include non-thermal components, thus a deep knowledge of the properties of these components and of their interplay with the thermal gas are needed to properly constrain the large-scale structure formation scenario. The discovery of diffuse cluster radio emission has represented an important step in the understanding of the physical processes in clusters of galaxies. Diffuse synchrotron sources are sensitive to the turbulence and shock structures of large-scale environments and provide essential complements to studies at other wavebands. Studies in the radio domain will fill essential gaps in both cluster astrophysics and in the growth of structure in the Universe, especially where the signatures of shocks and turbulence, or even the thermal plasma itself, may be otherwise undetectable.

The aim of this review is to present the observational results obtained in recent years in the radio domain related to the diffuse radio sources, in order to give an 
overview of the state of the art of the current knowledge of non-thermal cluster components. We will show how the radio properties can be linked to the X-ray properties and to the cluster evolutionary state, and will discuss the physical implications.

Cluster non-thermal emission of inverse-Compton (IC) origin is expected in the hard X-ray domain, due to scattering of the cosmic microwave background photons by the synchrotron relativistic electrons (Sarazin 1999). To present days, the detection of a non-thermal hard tail in the X-ray spectrum of clusters of galaxies has been reported in the literature (e.g. Fusco-Femiano et al. 1999, 2004, 2005, 2007, 2011; Rephaeli and Gruber 2002; Wik et al. 2009, 2011), and it is still debated in some cases. The presence of a high frequency non-thermal emission is clearly confirmed in the Ophiuchus cluster (Murgia et al. 2010b; Eckert 2011). The discussion of this topic is beyond the scope of this review, which is focused on the results in the radio band.

The organization of this paper is as follows: In Sect. 2 the general properties of diffuse cluster radio sources and their classification are presented; in Sect. 3 the problems related to the detection of diffuse emission are outlined; Sects. 4, 5, 6 give the observational properties of radio halos, relics and mini-halo, respectively. In Sect. 7 the current results about magnetic fields are presented. Section 8 presents the evidence for radio emission and magnetic fields beyond clusters of galaxies. Section 9 briefly summarizes the current models, and Sect. 10 depicts the future prospects.

The intrinsic parameters quoted in this paper are computed with $\Lambda \mathrm{CDM}$ cosmology with $H_{0}=71 \mathrm{~km} \mathrm{~s}^{-1} \mathrm{Mpc}^{-1}, \Omega_{m}=0.27$, and $\Omega_{\Lambda}=0.73$. Values taken from the literature have been scaled to this cosmology.

\section{Synchrotron emission and diffuse cluster radio sources}

Synchrotron emission is produced by the spiraling motion of relativistic electrons in a magnetic field. The power emitted by a relativistic electron depends on its energy (Lorentz factor) and on the magnetic field strength. The higher the magnetic field strength, the lower the electron energy needed to produce emission at a given frequency. The case of astrophysical interest is that of a homogeneous and isotropic population of electrons with a power-law energy distribution with index $\delta$. In this case, if the plasma is optically thin, as in diffuse cluster radio sources, the diagnostics of synchrotron radiation are the following:

(i) The emissivity is related to the number density of the relativistic electrons and to the intensity of the magnetic field, and the monochromatic emissivity follows a power-law with spectral index related to the index of the electron energy distribution $\alpha=(\delta-1) / 2$; typical observed radio spectra are around $0.7-0.8$, consistent with typical values of $\delta$ around 2.5 .

(ii) Energy losses of the radio emitting particles produce, with passing time, a change in the overall particle energy distribution, which in turn produces a modification of the emitted radio spectrum. In particular, the spectrum shows a cutoff at frequencies higher than a critical frequency $v^{*}$, related to the particle lifetime. Therefore aged radio sources show curved spectra and/or spectra steeper than the values $0.7-0.8$ indicated above. 
(iii) The synchrotron radiation from a population of relativistic electrons in a uniform magnetic field is linearly polarized, with the electric (polarization) vector perpendicular to the projection of the magnetic field onto the plane of the sky. The intrinsic polarization degree depends on the particle energy distribution, and equals $\sim 75-80 \%$ for typical values of the spectral index. Complex and disordered magnetic field structures decrease the observed degree of polarization.

(iv) The total energy content in a synchrotron radio source depends on the contributions from relativistic particles (electrons plus protons) and from magnetic fields (considering the fraction of the source volume occupied by magnetic fields, i.e. the filling factor). From radio data, it is possible to derive, the minimum value of the total energy, which occurs when the contributions of magnetic fields and relativistic particles are approximately equal (equipartition condition). The corresponding magnetic field is commonly referred to as equipartition value $B_{\text {eq }}$.

Rigorous treatments of the synchrotron theory and radiation processes are in $\mathrm{Pa}$ cholczyk (1970), Tucker (1975). Useful formulas of common use can be found in Govoni and Feretti (2004), Feretti and Giovannini (2008).

In recent years, there has been growing evidence for the existence of cluster largescale diffuse radio sources, of synchrotron origin, which have no optical counterpart and no obvious connection to the cluster galaxies, and are therefore associated with the ICM (Giovannini and Feretti 2002; Feretti and Giovannini 2008; Ferrari et al. 2008). The first diffuse radio source detected in a cluster of galaxies is the giant radio halo at the center of the Coma cluster (Large et al. 1959; Willson 1970). Several years later, extended diffuse emission was also detected at the periphery of the Coma cluster (Ballarati et al. 1981; Giovannini et al. 1991) and at the center of the Perseus cluster (Miley and Perola 1975; Noordam and de Bruyn 1982; Burns et al. 1992). Nowadays, diffuse radio emission with surface brightness down to $0.1 \mu \mathrm{Jy} \operatorname{arcsec}^{-2}$ at $1.4 \mathrm{GHz}$ is known in about 80 clusters, under several cluster evolutionary conditions (merging and relaxed clusters), at different cluster locations (center, periphery, intermediate distance), and on very different size scales: $100 \mathrm{kpc}$ to $>\mathrm{Mpc}$ (see Fig. 1 for several examples).

Diffuse sources are typically grouped in halos, relics and mini-halos (Feretti and Giovannini 1996), according to their location in the cluster and to the cluster type (merging or cool-core). The three classes will be extensively discussed in the next sections. We anticipate that halos are hosted in clusters showing merging processes (Feretti 2002; Giovannini and Feretti 2002) and are located at the cluster center. Relics are located in cluster peripheral regions of both merging and relaxed clusters. Prototypical examples of these two classes are both in the Coma cluster: the radio halo Coma $\mathrm{C}$ and the relic $1253+275$. Mini-halos are hosted in relaxed cool-core clusters, are centrally located, and usually surround a powerful radio galaxy; the prototype of this class is in the Perseus cluster. It should be remarked here that non-thermal diffuse emission can also be present beyond clusters, indicating the presence of relativistic particles and magnetic fields in very low density environments.

All diffuse radio sources in clusters have very steep radio spectra in common. A clear difference between halos and relics is in polarization property, with peripheral sources being more strongly polarized than the central ones. A possible problem in the classification of halos and relics could be represented by projection effects. 

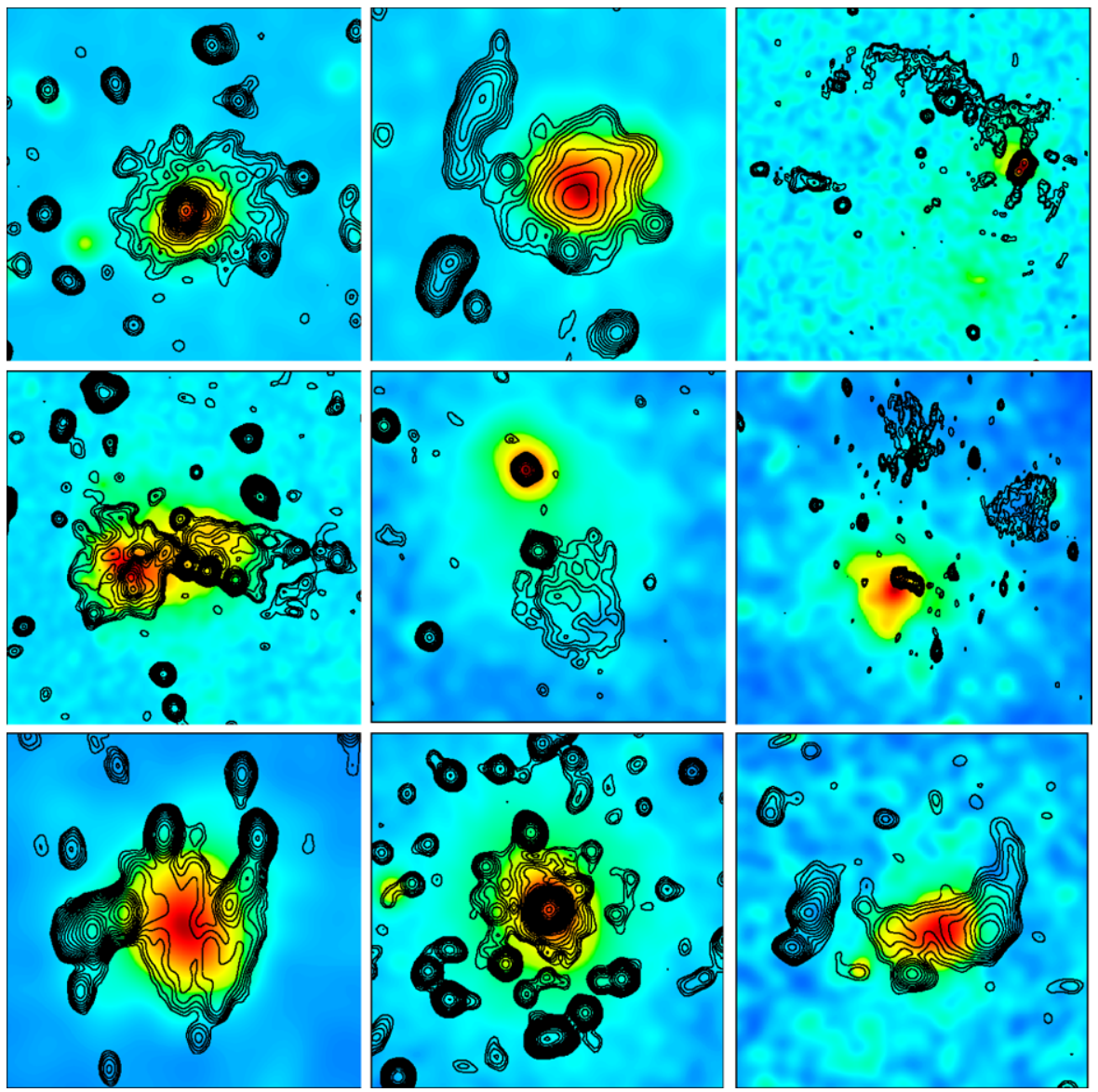

Fig. 1 Collection of clusters showing several types of radio emission, shown in contours, overlaid onto the X-ray emission, shown in colors. Clusters are (from left to right and from top to bottom) A 2219 (halo), A 2744 (halo + relic), A 115 (relic), A 754 (complex, halo plus relic), A 1664 (relic), A 548b (relic), A 520 (halo), A 2029 (mini-halo), RXCJ1314.4-2515 (halo plus double relics). For references to these objects see Tables 1, 3, and 5

In other words, halos could be actually located at cluster periphery, and simply projected onto the cluster center. This issue has been analyzed several years ago (Feretti 2002), using data at the time on about 30 clusters. From the distribution of projected distances of halos and relics from the cluster center, it was shown that there is a large excess of sources at the center, thus diffuse sources are not located at random position in clusters. This indicates that central halos are truly at the cluster center and not simply projected onto it. Moreover, detailed radio images show different morphologies of halos and relics. There are 42 halos currently known, while 39 clusters show at least 1 relic source (for a total of 50 relics). This confirms the findings above. A powerful way to distinguish between the two classes is through the polarization, this will become easy with new generation radio telescopes. 
Giovannini et al. (1999) showed that diffuse cluster sources are not present in all galaxy clusters, but are not a phenomenon as rare as formerly believed. They first showed that diffuse sources with the surface brightness limit of the NRAO VLA Sky Survey (NVSS Condon et al. 1998) are present in 6-9 \% of clusters with $L_{x}<$ $5 \times 10^{44} \mathrm{erg} / \mathrm{s}$, but are present in $27-44 \%$ of clusters with $L_{x}>5 \times 10^{44} \mathrm{erg} / \mathrm{s}$. This trend is confirmed for the occurrence of halos and relics, as reported in Sect. 4 and Sect. 5, respectively.

\section{Detection of diffuse emission}

For the detection of diffuse radio sources, the sensitivity to low surface brightness emission is the critical parameter. Single dish observations are in principle best suited to detect extended emission, however, they are generally limited by the high confusion, because of the large beam. Moreover, low angular resolution may prevent the separation of embedded unrelated discrete sources. On the other hand, an interferometer is characterized by a smaller observing beam, and thus by lower confusion, but its brightness sensitivity, set primarily by the aperture filling factor and the shortest spacings, may be insufficient. A proper sampling of the short spacings is vital not only for the detection of an extended source, but more crucially for the determination of the full size and the measurement of the source flux density.

Multi-frequency images with a similar uv-coverage are needed to derive spectral index distributions, which can provide information on the age and energy of radio emitting particles. The total spectral index can be easily obtained, but in many sources confusion problems do not allow a proper determination of low frequency fluxes.

To summarize, the instrument best suited to detect cluster diffuse radio sources should provide a compromise between high sensitivity to surface brightness, and high angular resolution. Moreover, since diffuse emission is generally characterized by steep radio spectra, the observations should be carried out at low frequencies ( $\lesssim 1.4 \mathrm{GHz}$ ). The Very Large Array (VLA) at $1.4 \mathrm{GHz}$ has been demonstrated to be a very good instrument to detect and study radio halos, owing to its superb sensitivity. However, it should be noted, as a caveat, that the Coma radio halo (see Fig. 2) is not detected by Westerbork Synthesis Radio Telescope (WSRT) and VLA observations at $1.4 \mathrm{GHz}$ because of the missing short spacings.

After the discovery of the Coma radio halo at $408 \mathrm{MHz}$ with the 250-ft radio telescope at Jodrell Bank (Large et al. 1959), confirmed from interferometric Cambridge One-Mile telescope radio data (Willson 1970), observations carried out mostly with single dish radio telescopes (Arecibo, Green Bank 300-ft, Effelsberg and others) and the WSRT found about 10 other clusters with a diffuse halo-type radio emission (see Hanisch 1982). Jaffe and Rudnick (1979), in their search for radio halos, found an extended emission region in the peripheral region of the Coma cluster near the strong radio source Coma A (3C 277.3). This was suggested to be a feature of the Coma cluster, in the following classified as a relic, thanks to observations at $408 \mathrm{MHz}$ with the Northern Cross Radio Telescope at better angular resolution (Ballarati et al. 1981). 


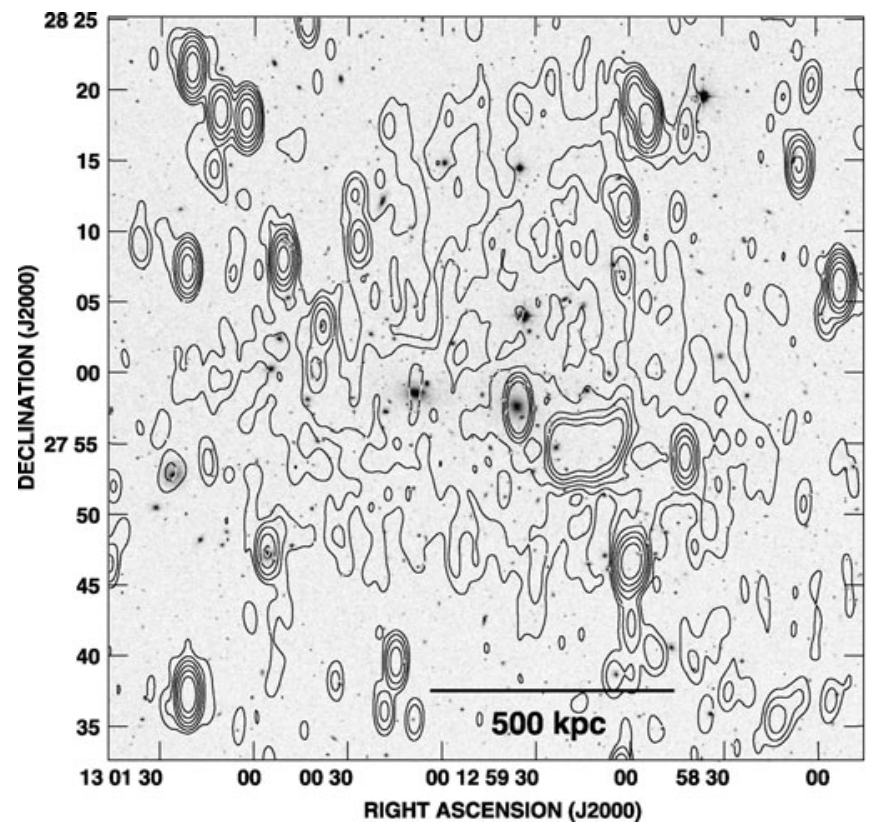

Fig. 2 Diffuse radio halo Coma $\mathrm{C}$ in the Coma cluster $(z=0.023)$ at $0.3 \mathrm{GHz}$ obtained with the WSRT, superimposed onto the optical image from the Digitized Sky Survey DSS1. The resolution of the radio image is $55^{\prime \prime} \times 125^{\prime \prime}\left(\right.$ FWHM, RA $\times$ DEC); contour levels are: 3, 6, 12, 25, 50, 100 mJy beam $^{-1}$

A significant breakthrough in the study of radio halos and other diffuse cluster radio sources was obtained thanks to the all-sky surveys with the VLA and WSRT. Giovannini et al. (1999), combining radio data from the NVSS at $1.4 \mathrm{GHz}$ and Xray catalogues, detected 18 new halo and relic candidates, in addition to the 11 already known, owing to the large beam and the good surface brightness sensitivity of the VLA D configuration. All new candidates were confirmed by more sensitive targeted observations, mostly performed with the VLA. Kempner and Sarazin (2001) presented seven new candidates from a search in the Westerbork Northern Sky Survey (WENSS; Rengelink et al. 1997) at 327 MHz; Rudnick and Lemmerman (2009) searched for Mpc-scale radio emission reprocessing WENSS radio images.

Recently, other extensive radio observations have been published. We note the Giant Metrewave Radio Telescope (GMRT) survey of massive galaxy clusters at $z=$ 0.2-0.4 by Venturi and collaborators (Venturi et al. 2007, 2008), who found diffuse sources in 10 clusters (out of which three new halos, two new core-halo sources and three new halo or relic candidates), the GMRT follow up at low frequencies of all clusters known to contain radio halos and relics up to $z=0.4$ (Giacintucci 2011), and the observing campaign carried out with the WSRT, VLA and GMRT by van Weeren et al. (2011a), who discovery six new radio relics, including a probable double system, and two radio halos. The above mentioned studies and their followups have produced rich information in a short time in this field. 

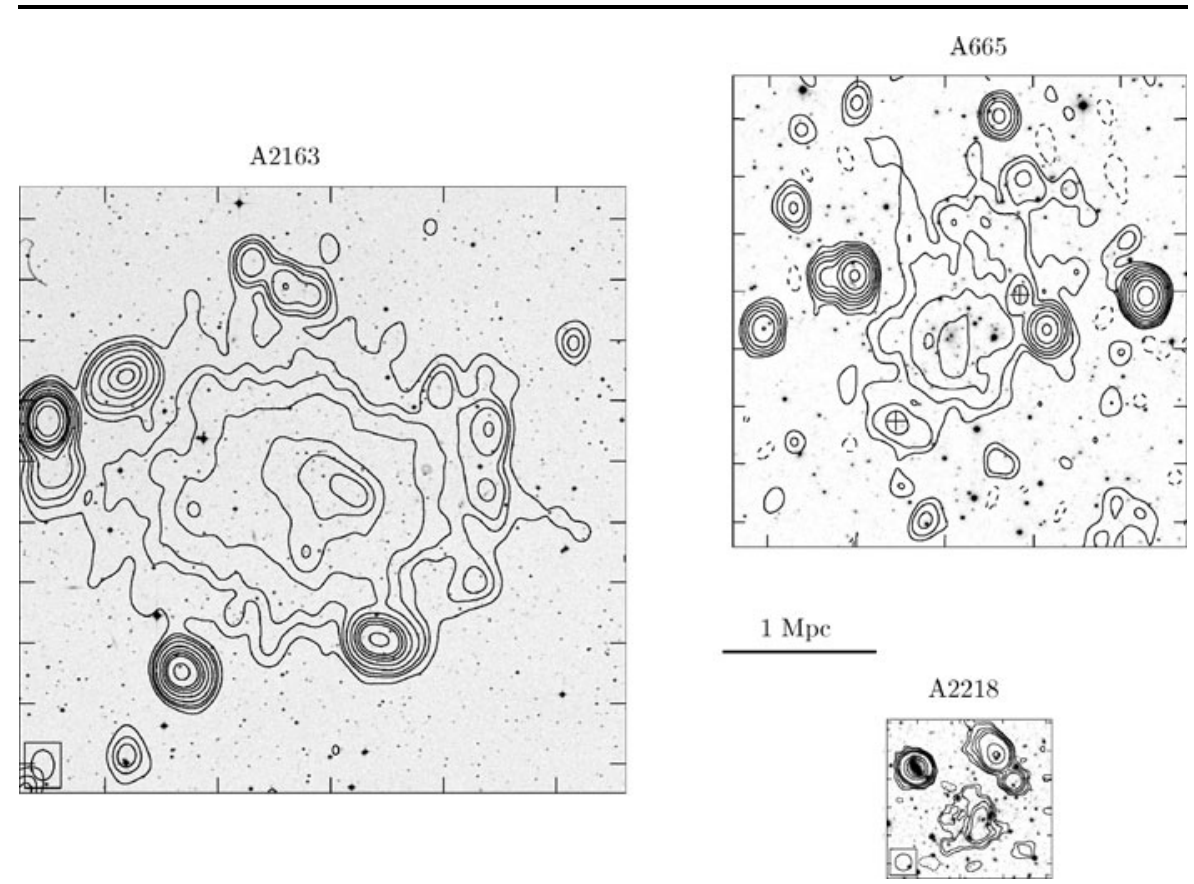

Fig. 3 Images of the clusters A 2163, A 665 and A 2218, hosting radio halos: radio emission is represented by contours, which are overlaid onto the optical image. The maps are all scaled to the same linear scale

\section{Radio halos}

Radio halos are diffuse radio sources of low surface brightness $\left(\sim 1-0.1 \mu \mathrm{Jy} \operatorname{arcsec}^{-2}\right.$ at $1.4 \mathrm{GHz}$ ) permeating the central volume of a cluster. They are typically extended with sizes of $\gtrsim 1 \mathrm{Mpc}$, regular in morphology, and are unpolarized down to a few percent level, probably because of internal or beam depolarization. The prototype of this class, Coma C at the center of the Coma cluster (Fig. 2) has been studied in detail by many authors (see e.g. Willson 1970; Giovannini et al. 1993; Kronberg et al. 2007; Brown and Rudnick 2011).

Thanks to the improvements in observations and data reduction procedures, radio halos with a smaller size and irregular morphology have also been detected in rich galaxy clusters. Their properties in most cases are similar to those of giant radio halos. Figure 3 presents images of well known radio halos of different sizes. A spectacular case is represented by the double merging system, A399 and A401, which both contain a radio halo and can be considered the only case so far of a double radio halo system (Murgia et al. 2010a, Fig. 4).

Table 1 reports all clusters known so far to host a radio halo as of September, 2011. This sample will be henceforth referred to as September2011-Halo collection. All halo clusters are undergoing merger processes, as demonstrated by the dynamical activity detected from X-ray and optical data (see Sect. 4.3.2).

The occurrence of halos in the NVSS sample (Giovannini et al. 1999) increases with the X-ray luminosity of the host clusters, reaching about $25 \%$ in clusters with 


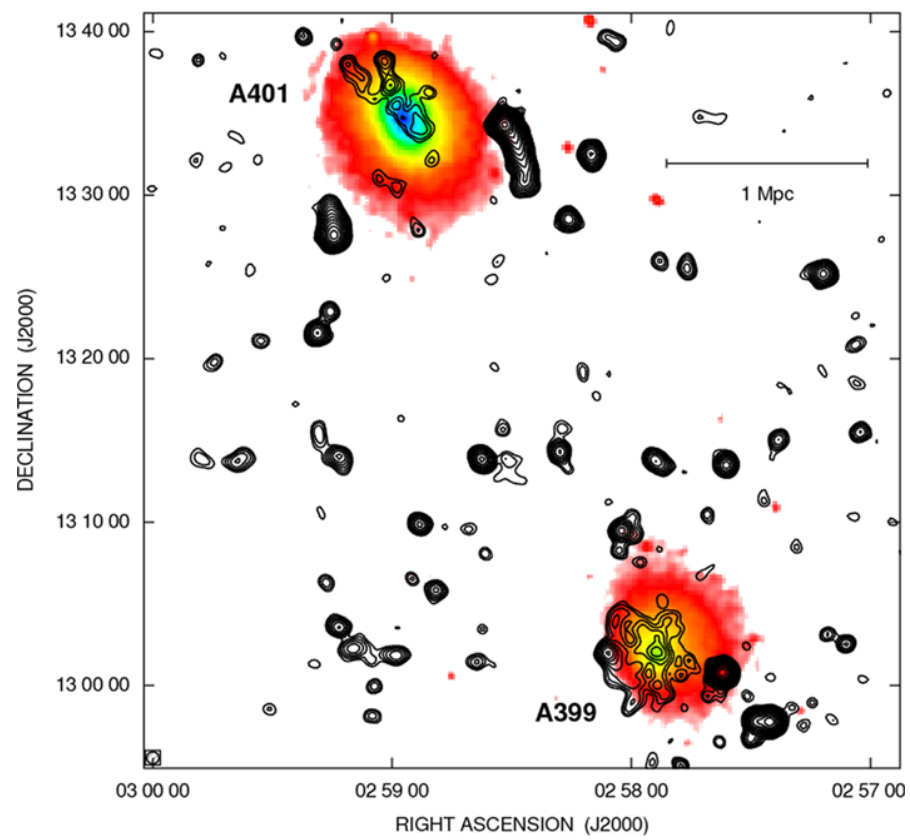

Fig. 4 Total intensity radio contours of the complex A401-A399. The radio image, obtained with the VLA at $1.4 \mathrm{GHz}$, is shown by the iso-contours and has a FWHM of $45^{\prime \prime} \times 45^{\prime \prime}$. The first contour level is drawn at $120 \mu \mathrm{Jy}_{\text {beam }}{ }^{-1}$ and the rest are spaced by a factor of $\sqrt{2}$. The color scale represents the XMM $\mathrm{X}$-ray image in the $0.2-12 \mathrm{keV}$ band

$L_{x}>5 \times 10^{44} \mathrm{erg} / \mathrm{s}$ (Giovannini and Feretti 2002). This trend is confirmed by the recent work of Cassano et al. (Cassano et al. 2011), who, with improved statistics, determined that radio halos are present in $\sim 30 \%$ of clusters with $L_{x}>5 \times 10^{44} \mathrm{erg} / \mathrm{s}$.

Figure 5 reports the redshift distribution of clusters with a radio halo. This distribution is rather homogeneous up to $z=0.35$. The scarce number of halos beyond this value is likely due to sensitivity limitations, and/or selection effects (Giovannini et al. 2009), although a real lack of high redshift radio halos cannot be excluded. This might possibly be related with the cluster evolution, or with strong Inverse Compton losses with the cosmic microwave background radiation. There are currently three high redshift $(z>0.4)$ clusters known to host radio halos: A851 at $z=0.4069$ (Giovannini et al. 2009), CL 0016+16 (Giovannini and Feretti 2000), and MACS J0717.5+3745 (Bonafede et al. 2009b; van Weeren et al. 2009a), both at $z=0.55$. The latter shows also significant polarization, as also found in A 2255 (Govoni et al. 2005; Pizzo et al. 2011), unlike most radio halos which are unpolarized.

The physical parameters in radio halos can be estimated assuming equipartition conditions, and further assuming equal energy in relativistic protons and electrons, a volume filling factor of 1 , a low frequency cutoff of $10 \mathrm{MHz}$, and a high frequency cutoff of $10 \mathrm{GHz}$. The derived minimum energy densities are of the order of $10^{-14}$ $10^{-13} \mathrm{erg} \mathrm{cm}^{-3}$, i.e. much lower than the energy density in the thermal gas. The corresponding equipartition magnetic field strengths range from 0.1 to $1 \mu \mathrm{G}$. 


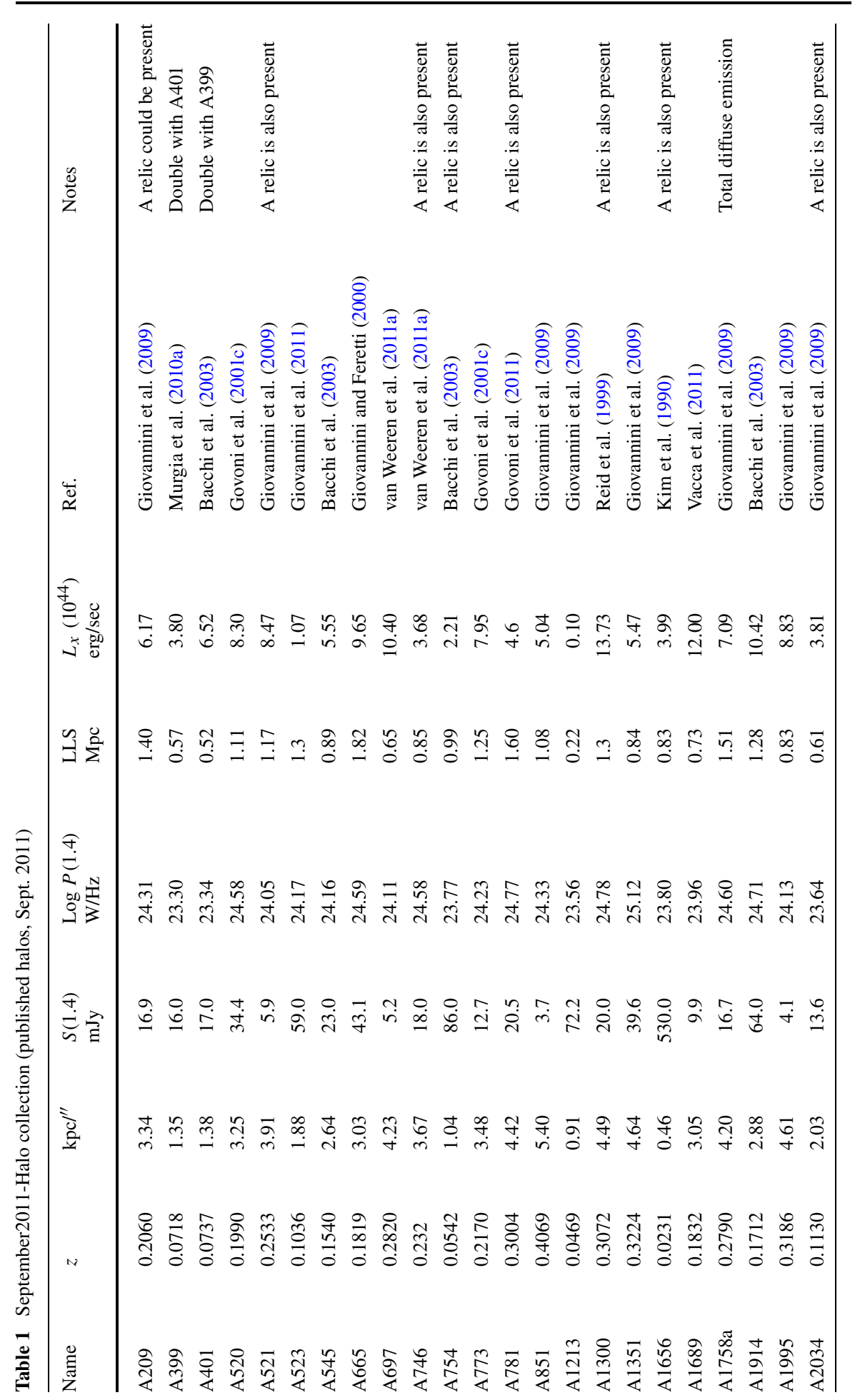




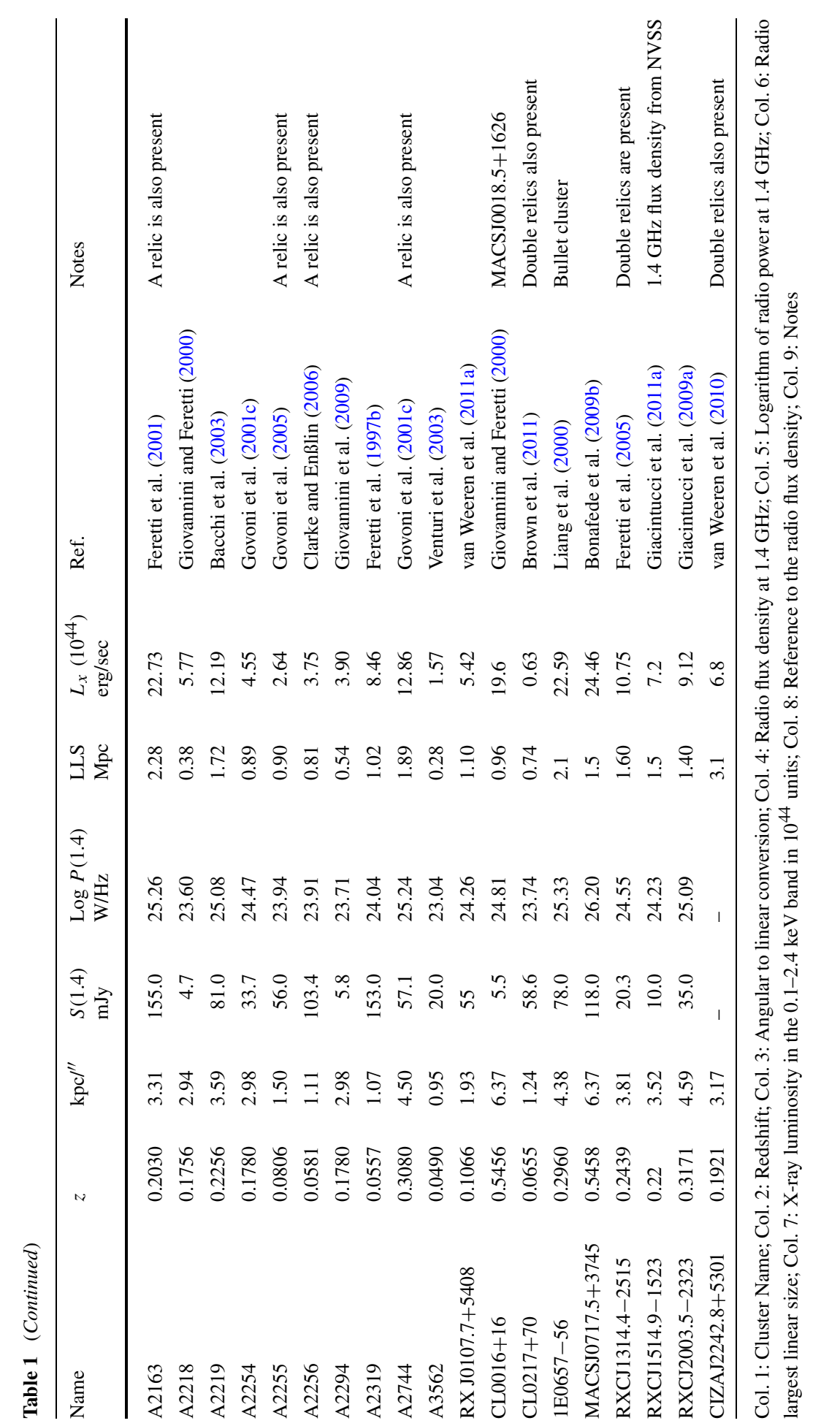


Fig. 5 Distribution of known clusters with radio halos as a function of redshift

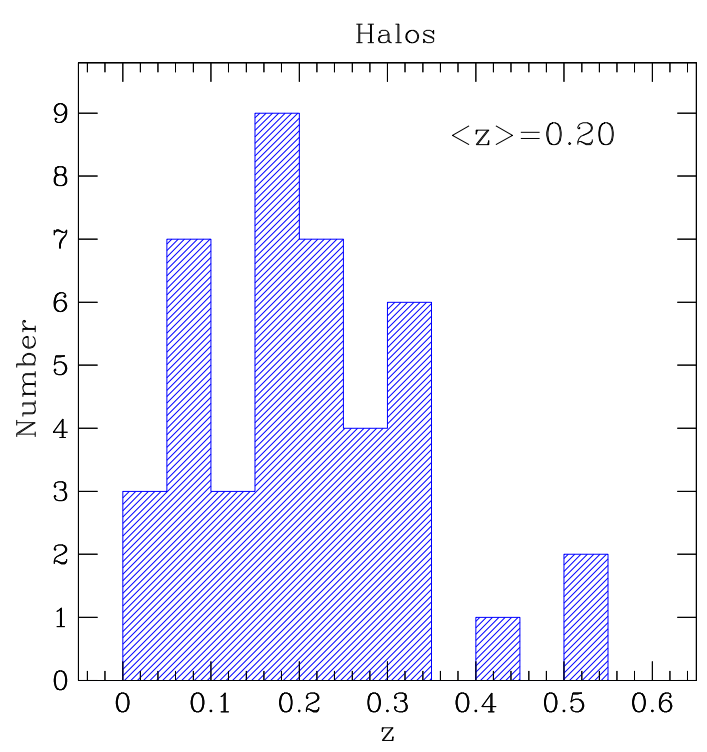

\subsection{Spectra of radio halos}

\subsubsection{Integrated spectrum}

The integrated radio spectra of halo sources are still poorly known. The difficulty of spectral studies are that (i) only in a few cases the spectrum is obtained with more than three flux density measurements at different frequencies, (ii) for most sources the highest available frequency is $1.4 \mathrm{GHz}$, therefore it is difficult to determine the presence of a spectral steepening, crucial to discriminate between different reacceleration models.

Spectral data available on the objects of the September2011-Halo collection are reported in Table 2, where we arrange the information according to the number of available frequency measurements. Halos always show a steep spectrum $(\alpha \gtrsim 1){ }^{1}$ The best studied integrated spectrum is that of the Coma cluster where clear evidence of a high frequency steepening is present (see Thierbach et al. 2003 for a detailed discussion). Indications of high frequency spectral steepening are reported in 4 more cases. A very steep spectrum is shown by the radio halo in A1914 (Bacchi et al. 2003) where nine different points show a straight spectrum with $\alpha=1.88$, although a possible high frequency curvature has been suggested (Komissarov and Gubanov 1994).

Halo spectra are typical of aged radio sources (see Sect. 2). In general, it is estimated that the radiative lifetime of relativistic electrons from synchrotron and inverse Compton energy losses is of the order of $\sim 10^{8}$ yr (Sarazin 1999). Since the expected diffusion velocity of the electron population is of the order of the Alfvén speed $\left(\sim 100 \mathrm{~km} \mathrm{~s}^{-1}\right)$, the radiative electron lifetime is too short to allow the particle

${ }^{1} S(v) \propto v^{-\alpha}$ is assumed throughout the paper. 
Table 2 Spectral index of radio halos. The cluster data are grouped according to how many flux density measurements at different frequencies are available for the spectrum determination: the first group reports clusters with more than three frequency measurements, the second group reports clusters with three frequency measurements, the third group reports clusters with two frequency measurements

\begin{tabular}{llll}
\hline Name & $\alpha 1$ & $\alpha 2$ & $\begin{array}{l}\text { Temperature } \\
\text { keV }\end{array}$ \\
\hline
\end{tabular}

$\mathrm{keV}$

\begin{tabular}{|c|c|c|c|c|}
\hline A1656 & $\alpha_{0.31}^{1.4}=1.16$ & $\alpha_{1.4}^{4.8}=2.28$ & 8.4 & Thierbach et al. (2003) \\
\hline A1914 & $\alpha_{0.26}^{1.4}=1.88$ & & 7.9 & Bacchi et al. (2003) \\
\hline A2256 & $\alpha_{0.22}^{1.4}=1.61$ & & 6.6 & Brentjens (2008) \\
\hline A 3562 & $\alpha_{0.3}^{0.8}=1.3$ & $\alpha_{0.8}^{1.4}=2.1$ & 5.2 & Giacintucci et al. (2005) \\
\hline $1 \mathrm{E} 0657-56$ & $\alpha_{0.84}^{5.9}=1.3$ & & 10.6 & Liang et al. (2000) \\
\hline A521 & $\alpha_{0.24}^{1.4}=1.8$ & & 5.9 & $\begin{array}{l}\text { Giovannini et al. (2009) and } \\
\text { references therein }\end{array}$ \\
\hline A754 & $\alpha_{0.07}^{0.3}=1.1$ & $\alpha_{0.3}^{1.4}=1.5$ & 9.5 & Bacchi et al. (2003) \\
\hline A 2163 & $\alpha_{0.3}^{1.3}=1.1$ & $\alpha_{1.3}^{1.6}=1.5$ & 13.3 & Feretti et al. (2001) \\
\hline A2319 & $\alpha_{0.4}^{0.6}=0.9$ & $\alpha_{0.6}^{1.4}=2.2$ & 8.8 & Feretti et al. (1997b) \\
\hline RXCJ2003.5-2323 & $\alpha_{0.2}^{1.4}=1.3$ & & - & Venturi et al. (2009) \\
\hline A545 & $\alpha_{1.3}^{1.6}>1.4$ & & 5.5 & Bacchi et al. (2003) \\
\hline A665 & $\alpha_{0.3}^{1.4}=1.0$ & & 9.0 & Giovannini and Feretti (2000) \\
\hline A697 & $\alpha_{0.3}^{1.4}=1.5$ & & 10.2 & $\begin{array}{l}\text { Giovannini et al. (2009), } \\
\text { van Weeren et al. (2011a), Macario } \\
\text { (2011) }\end{array}$ \\
\hline A1300 & $\alpha_{0.3}^{1.4}=1.3$ & & 9.2 & Giacintucci (2011) \\
\hline A1758 & $\alpha_{0.3}^{1.4}=1.5$ & & 7.1 & Giacintucci (2011) \\
\hline A 2218 & $\alpha_{1.4}^{5.0}=1.6$ & & 7.1 & Giovannini and Feretti (2000) \\
\hline A2219 & $\alpha_{0.3}^{1.4}=0.9$ & & 12.4 & Orrù et al. (2007) \\
\hline A 2255 & $\alpha_{0.3}^{1.4}=1.7$ & & 6.9 & Feretti et al. (1997a) \\
\hline A 2744 & $\alpha_{0.3}^{1.4}=1.0$ & & 10.1 & Orrù et al. (2007) \\
\hline MACSJ0717.5+3745 & $\alpha_{1.4}^{4.7}=1.3$ & & 11 & $\begin{array}{l}\text { Bonafede et al. }(2009 b) \text {, and } \\
\text { references therein }\end{array}$ \\
\hline $\mathrm{CLO} 217+70$ & $\alpha_{0.3}^{1.4}=1.2$ & & - & Brown et al. (2011) \\
\hline RXCJ1514.9-1523 & $\alpha_{0.3}^{1.4}=1.6$ & & - & Giacintucci et al. (2011a) \\
\hline
\end{tabular}

Col. 1: Cluster name; Col. 2: Total spectral index value for straight spectra, or low frequency spectral index value for curved spectra; Col. 3: High frequency spectral index value for curved spectra; Col. 4: Average cluster temperature in $\mathrm{keV}$ from literature data; Col. 5: References to spectral index information

diffusion throughout the cluster volume. Thus, the radiating electrons cannot have been produced at some localized points of the cluster. They must undergo in situ energization, with an efficiency comparable to the energy loss processes (Petrosian 2001), or be continuously injected into the cluster volume (Jaffe 1977). In Sect. 4.3.2, we will highlight that recent cluster mergers are likely to supply energy to the halos, through the reacceleration of radio emitting electrons. 
In the framework of the link between halos and clusters mergers, the existence of a possible correlation between radio spectral index and cluster temperature has been investigated in the literature (Feretti et al. 2004a; Giovannini et al. 2009), and marginal evidence that clusters at higher temperature tend to host halos with a flatter spectrum has been found. By using the most recent results (September2011-Halo collection), and considering the average spectral index value in the frequency range $0.3-1.4 \mathrm{GHz}$, it is found that:

- radio halos in clusters with an average temperature less than $8 \mathrm{keV}$ show an average spectral index of $1.7 \pm 0.2$;

- radio halos in clusters with a temperature in the range from 8 to $10 \mathrm{keV}$ show an average spectral index of $1.4 \pm 0.4$;

- halos in clusters with a temperature greater than $10 \mathrm{keV}$ show an average spectral index of $1.2 \pm 0.2$.

This trend indicates that hotter clusters are likely to host halos with flatter spectra, i.e. halos where energy gains are more significant. Unfortunately, the statistics is still poor, because of the low number of data and highly inhomogeneous spectral index measurements. This result, if confirmed, is a very important clue in favor of reacceleration models: indeed hottest clusters are more massive and undergoing more violent mergers, thus supplying more energy to the radio emitting particles (Feretti et al. 2004b, 2004c; Cassano and Brunetti 2005; Cassano et al. 2008).

\subsubsection{Spectral index distribution}

A spectral index image is a powerful tool to understand the physical properties of radio halos, since it reflects the variations of the shape of the electron energy distribution and of the magnetic field in which they emit.

The first spectral index map of a radio halo was obtained for the Coma cluster (Giovannini et al. 1993), where the high sensitivity of images allowed the computation of spectral index up to $\sim 30^{\prime}$ from the cluster center. The spectral distribution is smooth with a steepening from the center to the peripheral regions, where the spectral index is $\alpha \sim 2$. The spectral index images of giant radio halos in A665 and A2163, with an angular resolution $\sim 1^{\prime}$, show flattening and patches, and radial steepening in the undisturbed cluster regions (Feretti et al. 2004b).

In the bullet cluster 1E0657-56, no evidence of spectral steepening in the outer regions of the radio halo is found (Liang et al. 2000). The spectral index distribution in the A3562 radio halo shows a very complex structure, with an average value of $\alpha \sim 1.5$ and a number of knots steepening up to $\alpha \sim 2$ (Giacintucci et al. 2005). The spectral index images of A2744 and A2219 (Orrù et al. 2007) show the presence of localized regions in which the radio spectrum is significantly different from the average. From the comparison of the spectral index map of A2744 (Fig. 6, left panel) and the X-ray data from Chandra, it is found for the first time that the flat spectrum regions of the radio halo tend to have higher temperature (Fig. 6, right panel).

In the radio halo of A2255, the spectrum is steeper at the center and flatter at the locations of the radio filaments (Pizzo and de Bruyn 2009), indicating either that we are looking at a superposition of different structures (filaments in the foreground plus 

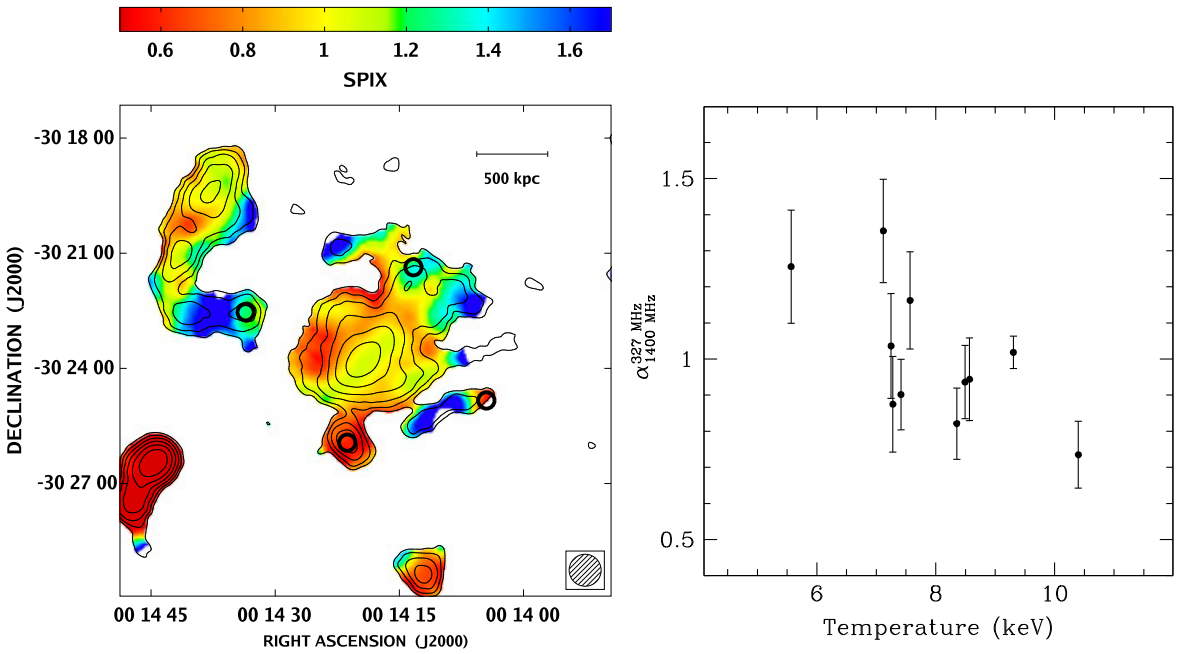

Fig. 6 Left panel: Spectral index image of A2744 between $325 \mathrm{MHz}$ and $1.4 \mathrm{GHz}$, with a resolution of $50^{\prime \prime} \times 50^{\prime \prime}$. Circles indicate the positions of discrete sources. The radio halo is at the center, while the elongated feature to the NE is the cluster relic. Right panel: Plot of the radio halo spectral index between $325 \mathrm{MHz}$ and $1.4 \mathrm{GHz}$ versus gas temperature, obtained by the Chandra temperature image from Kempner and David (2004). The values of the spectral index and of temperature have been derived in a grid of boxes of about $1^{\prime}$ in size. Both panels are from Orrù et al. (2007)

real halo in the background) seen in projection across the cluster center, or that the halo is intrinsically peculiar. The complex cluster A2256 shows flat spectral index in the region NW of the cluster center, and steep spectral index regions toward the SE of the cluster center (Kale and Dwarakanath 2010). The authors interpret this spectral behavior as resulting from two populations of relativistic electrons created at two epochs (two mergers).

Spectral variations are likely to reflect the energy losses/gains of the radiating electrons. It can be shown (Feretti et al. 2004b) that, in regions of identical volume and identical radio brightness, a flattening of the spectral index from 1.3 to 0.8 requires an amount of energy injected into the electron population larger by a factor of $\sim 2.5$. Alternatively, if electrons are no longer gaining energy, a flatter spectrum would indicate that the last reacceleration is more recent. Therefore, it can be argued that the radio spectrum is flatter in the regions influenced by merger processes. The data available so far are in support of electron reacceleration models, with energy supplied by cluster merger (Cassano 2010). In particular the spectral radial steepening is interpreted as due to the combined effect of a radial decrease of the cluster magnetic field strength and of the presence of a high-energy break in the energy distribution of the reaccelerated electron population (Brunetti et al. 2001).

\subsubsection{Ultra-steep spectrum halos}

In recent years thanks to the improvement in low frequency radio observations, a large attention has been devoted to the search for and discussion of ultra-steep spectrum radio halos (see e.g. Venturi 2011). 
From the synchrotron emission theory, it is derived that strong energy losses in the radiating electrons produce a cutoff at high frequency, while the radio spectrum at low frequency reflects the original energy distribution of electrons. The existence of radio halos showing very steep spectra also in the low frequency range would indicate objects characterized by dramatic energy losses. Cassano (2010) argues that radio halos with spectral index $\alpha \sim 1.7$, and steeper, are likely missed by present observations at $\mathrm{GHz}$ frequencies. This radio halo population can only be revealed with highly sensitive observations at very low radio frequencies.

Table 2 shows that only a few ultra-steep spectrum radio halos are currently known: A1914 (Bacchi et al. 2003), A521 (Dallacasa et al. 2009; Giovannini et al. 2009), and A2255 (Feretti et al. 1997a). The cluster A697 was previously classified among ultra-steep spectrum halos (Macario et al. 2010), however, according to recent flux measurements (van Weeren et al. 2011a) the spectral index is around 1.5. These objects may be the tip of the iceberg of a population which should be detected by future instruments at low frequencies (LOFAR, LWA, SKA). We note that the few ultra-steep radio halos are in relatively low temperature clusters, in agreement with the correlation between radio halo spectral index and cluster temperature (see Sect. 4.1.1).

\subsection{Halo size}

Classical halos, i.e. the first which have been detected, are giant radio sources of about $1 \mathrm{Mpc}$, or more, in size. In recent years, however, several small-size halos have been revealed down to few hundred kpc, owing to the improvement in sensitivity and angular resolution of instruments. Giovannini et al. (2009) reported the correlation between the radio halo linear size and the total radio power, and discussed that this correlation is continuous from small halos up to giant Mpc-scale radio halos. The correlation obtained for the September2011-Halo collection is presented in Fig. 7.

Fig. 7 Monochromatic radio power of halos at $1.4 \mathrm{GHz}$ versus their largest linear size (LLS) measured at the same frequency. Names of peculiar objects are indicated (see text)

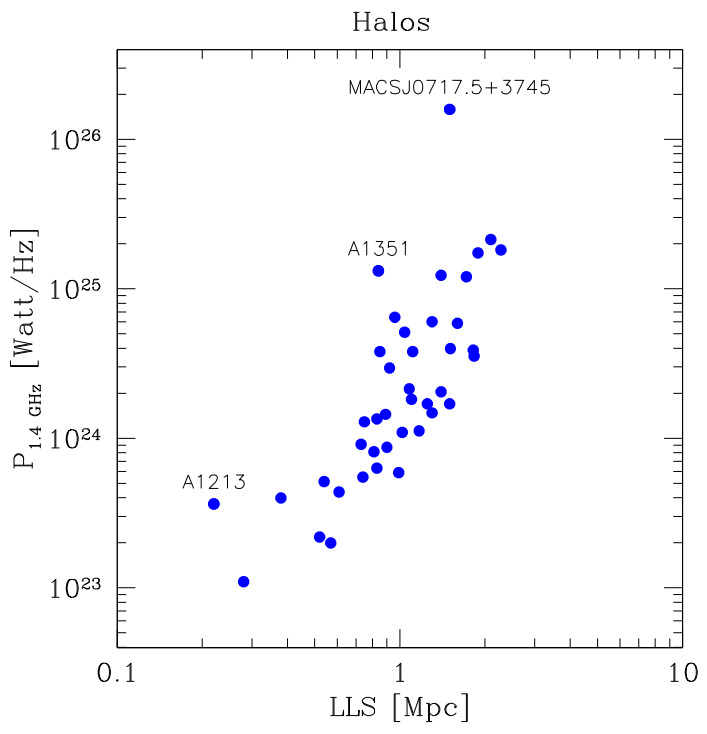


The slope of the correlation is in agreement with results obtained using only giant radio halos by Cassano et al. (2007). Murgia et al. (2009) fitting the azimuthally averaged brightness profile with an exponential, found that radio halo emissivity is remarkably similar from one halo to the other, despite quite different length-scales, in agreement with the correlation in which larger halos show a higher radio power (see the discussion in Sect. 4.3). Possible evolutionary effects with redshift cannot be analyzed with the current data, because of poor statistics.

There are 3 outliers with respect to the general correlation: one is MACS J0717.5+3745 (Bonafede et al. 2009b), which is the most powerful radio halo, but not the most extended; the second is the halo in A1351, which shows a size smaller than expected from its radio power. It is an irregular radio halo (Giovannini et al. 2009; Giacintucci et al. 2009b) and could be related to a recent merger. The third one is the so far smallest radio halo, located in the poor Abell cluster A1213 (see also Sect. 4.3.1). As discussed in Giovannini et al. (2009), more data are necessary to better understand its properties.

Except for the few cases outlined above, the correlation of radio size versus radio power shows a small dispersion, confirming that giant and smaller radio halos belong to the same class of sources: halos as small as a few hundred kpc show the same properties as Mpc size giant halos. This is in support of a common origin and physical mechanism for giant and small-size radio halos.

\subsection{Radio-X-ray connection}

The radio properties of halos are linked to the cluster properties. The probability of detecting radio halos is highest in the clusters with the highest X-ray luminosity (see Sect. 4). Moreover, the monochromatic radio power of a halo at $1.4 \mathrm{GHz}$ correlates with the cluster X-ray luminosity, and mass and temperature. In Fig. 8 we present

Fig. 8 Monochromatic radio power of radio halos at $1.4 \mathrm{GHz}$ versus the cluster X-ray luminosity between 0.1 and $2.4 \mathrm{keV}$, obtained for the merging clusters with radio halos. Names of peculiar objects are indicated (see text)

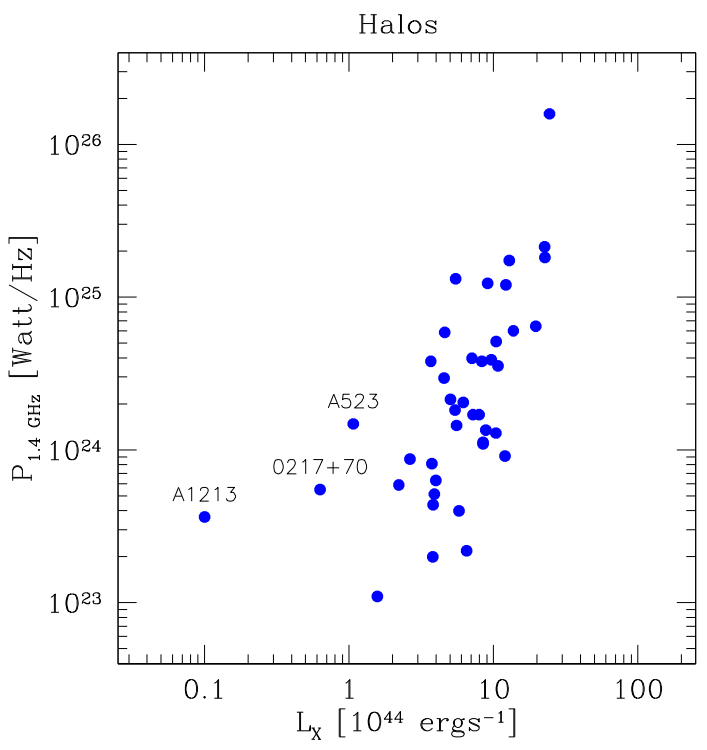


the correlation between the halo radio power at $1.4 \mathrm{GHz}$ and the X-ray luminosity between $0.1-2.4 \mathrm{keV}$. We note that the correlation is obtained for merging clusters with radio halos and cannot be generalized to all clusters (see Sect. 4.3.2).

The most powerful radio halo is in the cluster MACS J0717.5+3745. It is remarkable that its radio power is about an order of magnitude higher than that of the second most powerful known halo. In the low X-ray luminosity regime, there are a few clusters well above the extrapolation of the correlation, which will be discussed in the next subsection. If we exclude the three outliers named in Fig. 8 we find $P_{1.4} \propto L_{x}^{2}$. On the other hand, ultra-steep spectrum radio halos, discussed in Sect. 4.1.3, are expected to be less luminous than predicted by the radio-X-ray correlation, according to Cassano (2010).

An extrapolation of the above correlation to low radio and X-ray luminosities indicates that clusters with $L_{x} \lesssim 10^{44} \mathrm{erg} \mathrm{s}^{-1}$ should host halos of power $<10^{23} \mathrm{~W} \mathrm{~Hz}^{-1}$. With a typical size of $1 \mathrm{Mpc}$, they would have a radio surface brightness lower than current limits obtained in the literature. It should be stressed, however, that the less powerful radio halos are also smaller in size (Fig. 7), implying that actually it may not be appropriate to assume a typical size $\gtrsim 1 \mathrm{Mpc}$ for low power radio halos.

Brunetti et al. $(2007,2009)$ report the same correlation using all clusters searched for radio halos with the GMRT and derived upper limits to the radio power of undetected halos, by adopting a halo size of $1 \mathrm{Mpc}$. They found that upper limits are all in the region of high X-ray luminosity, and are all about one order of magnitude below the correlation, indicating a difference between clusters with and without halos. Their conclusion is that the distribution is likely related to the dynamical state of the cluster (see Sect. 4.3.2): radio halos are associated with dynamically disturbed clusters, while upper limits refer to relaxed clusters. This is in agreement with the result that halos are only present in merging clusters. In Fig. 8 only the clusters with halos are reported, thus no upper limits are shown.

Since cluster X-ray luminosity and mass are correlated (Reiprich and Böhringer $2002)$, the correlation between radio power $\left(P_{1.4 \mathrm{GHz}}\right)$ and X-ray luminosity could reflect a dependence of the radio power on the cluster mass $M$. A correlation of the type $P_{1.4 \mathrm{GHz}} \propto M^{2.3}$ has been derived (Govoni et al. 2001c; Feretti 2003), where $M$ is the total gravitational mass within a radius of $3\left(H_{0} / 50\right)^{-1} \mathrm{Mpc}$. Using the cluster mass within the viral radius, the correlation is steeper (Cassano et al. 2006, 2007).

Another link between radio and X-ray properties is suggested by the close similarity of the radio and X-ray structure found in a number of well resolved clusters. The similarity was quantitatively confirmed (see e.g. Govoni et al. 2001b; Feretti et al. 2001; Giacintucci et al. 2005), by comparing the point-to-point surface brightness of the radio and X-ray emission. A nearly linear relationship was found in three clusters (A2255, A2744 and A3562), while a power-law relation with index $<1$ is present in Coma, A2319 and A2163. As discussed in Govoni et al. (2001b), the correlation suggests a direct connection between the thermal X-ray plasma and the non-thermal radio plasma; its slope is that expected by electron reacceleration models.

The radio-X-ray brightness relation discussed above is valid for giant and regular halos. Actually, we have noticed that, owing to recent deep observations, more irregular and asymmetric halos have been found. In these halos, the radio emission may 

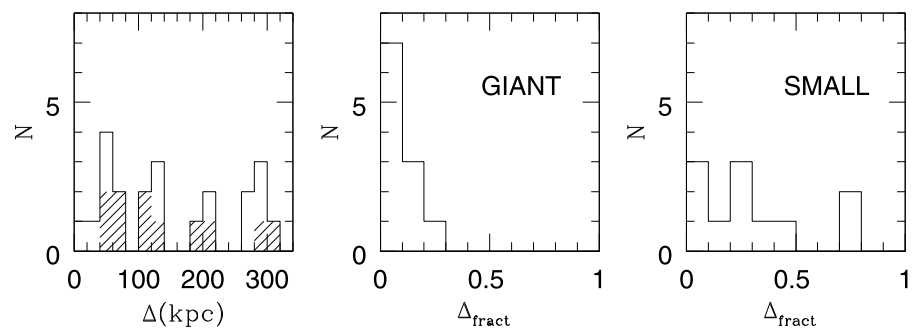

Fig. 9 Left panel: Values of the offset $\Delta$ between the radio and X-ray centroids in kpc. The dashed area refers to giant halos. Middle and right panels: Fractional offset ( $\Delta$ /radio halo size) for giant halos (size $\geq 1 \mathrm{Mpc}$ ) and small halos (size $<1 \mathrm{Mpc}$ ), respectively

show significant displacement from the X-ray emission. This has been investigated by comparing the center of the radio halo with that of the X-ray gas distribution in a sample of clusters with good radio and X-ray data (Feretti et al. 2010). The left panel of Fig. 9 shows that both giant and small radio halos can be significantly shifted, up to hundreds kpc, with respect to the centroid of the host cluster. To highlight radio halos with the most pronounced asymmetric distribution, the ratio between the radio-X-ray offset and the halo size is plotted in the middle and right panels of Fig. 9. It is deduced that halos can be quite asymmetric with respect to the X-ray gas distribution, and this becomes more relevant when halos of smaller size are considered. A possibility is that the asymmetry in the structure is caused by magnetic field fluctuations as large as hundreds of kpc, as suggested by Vacca et al. (2010) on the basis of magnetic field modeling.

\subsubsection{Halos in poor clusters}

Giovannini et al. (2009) were the first to discuss the possible presence of a radio halo in A1213, an under-luminous X-ray cluster i.e. well outside the correlation between radio power and X-ray luminosity. A similar case is found in the cluster CLO217+70 by Brown et al. (2011). Clear evidence of a radio halo more luminous than predicted by the radio-X-ray correlation is detected in A523 (Giovannini et al. 2011).

The number of these cases is still low, and some observational uncertainties may be present (see Giovannini et al. 2011 for a more detailed discussion), however, there is observational evidence that in a few cases giant radio halos can be associated with relatively low luminosity X-ray clusters, i.e. low mass clusters and low density environments. These halos are over-luminous in radio by at least an order of magnitude with respect to what is expected from the extrapolation of the observed radio power$\mathrm{X}$-ray luminosity. These objects are likely to represent a new class of halos that are difficult to explain by classical radio halo models. We note that ultra-steep spectrum halos, presented in Sect. 4.1.3 and predicted by reacceleration models, show the opposite behavior, i.e. they should be less luminous in radio than predicted by the radio-X-ray correlation. Giovannini et al. (2011) suggest that these powerful radio halos associated with low density environments could be either young halos or related to clusters at a special time of the merger event, when particle acceleration processes have a higher efficiency (see e.g. Brunetti and Lazarian 2011b). Another 
possibility is that the X-ray luminosity might not be a good indicator of the previous history of cluster merging activity in these cases.

\subsubsection{Halos and cluster mergers}

Circumstantial evidence of the link between cluster merging and radio halos has been outlined in early works on this topic (see e.g. Feretti 1999, 2002; Giovannini and Feretti 2002; Schuecker et al. 2001; Böhringer and Schuecker 2002), on the basis of the presence of radio halos only in clusters showing X-ray and substructure, X-ray temperature gradients and weak (or absent) cooling flow. This has been strongly supported by optical data (see e.g. Girardi and Biviano 2002; Ferrari et al. 2003; Boschin et al. 2004, 2006). The first quantitative analysis of the cluster dynamical state was performed by Buote (2001), who obtained the cluster dipole ratio from X-ray images and found that the strongest radio halos appear only in those clusters experiencing the largest departures from a virialized state. Cassano et al. (2010) recently confirmed the connection between radio halos and cluster mergers, from a quantitative study on a larger sample, using Chandra X-ray data and adopting three methods to characterize the cluster state, i.e. the power ratio, the centroid shift and the X-ray brightness concentration parameter.

The link between radio halos and cluster merger is well supported by several arguments, and recent findings obtained with improved statistics support the early suggestion that there is a clear separation between relaxed and non-relaxed clusters, in the radio behavior. The bi-modality between halo and non-halo clusters, discussed by Brunetti et al. (2009), reflects the dichotomy existing in the evolutionary state of clusters, between non-relaxed and relaxed clusters, as also discussed by Rossetti et al. (2011).

What is intriguing is the existence of dynamically disturbed clusters which do not show any evidence of radio halo. Cassano et al. (2010) find four such clusters: A141, A781, A2631 and MACS J2228.5+2036. A 781 has been found to host a radio halo (Govoni et al. 2011), but there are still three outliers (out of them one cluster is at $z>0.32$ ). Some other well known merging clusters do not host a radio halo, e.g. A119 (Giovannini and Feretti 2000) and A2146 (Russel et al. 2011).

The existence of merging clusters with halos, and merging clusters without halo, indicates that there is a dichotomy in merging clusters, which is not currently understood. It may be possible that giant halos are only present above a threshold of mass or temperature. Future radio data with next generation instruments (LOFAR, LWA, SKA) will allow the detection of low brightness/low power large halos, in order to clarify if halos are present in all merging clusters or only in the most massive ones. The dichotomy has to be solved to get a full and comprehensive understanding of these phenomena.

\section{Relics}

Relic sources are diffuse extended sources similar to radio halos in their low surface brightness, large size ( $\gtrsim 1 \mathrm{Mpc})$ and steep spectrum $(\alpha \gtrsim 1)$, but, unlike halos, they are located in cluster peripheral regions and are strongly polarized $(\sim 20-30 \%)$. 


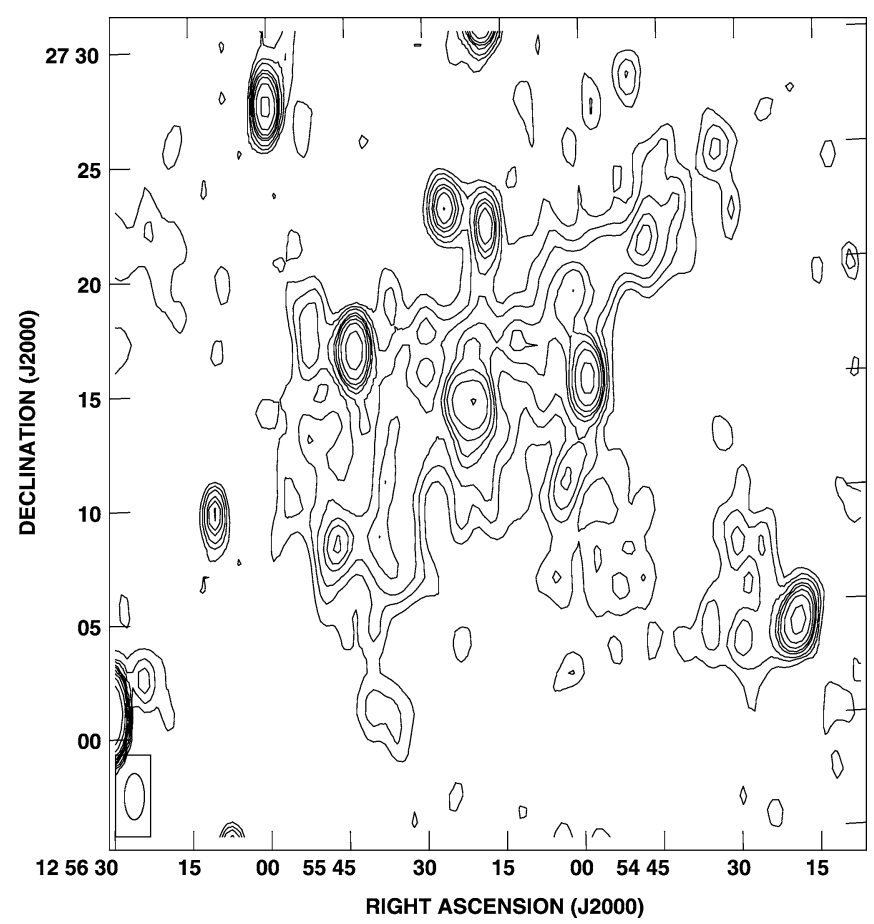

Fig. 10 Image at $327 \mathrm{MHz}$, obtained with the WSRT, of the relic $1253+275$ in the Coma Cluster. The beam is $50^{\prime \prime} \times 120^{\prime \prime}(\mathrm{RA} \times \mathrm{DEC})$. Contour levels are 3, 5, 7, 10, 12, 15, 20, 30, $50 \mathrm{mJy} \mathrm{beam}^{-1}$. The cluster center is at about 1.2 Mpc distance in the NE direction. See Giovannini et al. (1991) for more details

Observations of relics provide the best indications for the presence of $\mu \mathrm{G}$ level magnetic fields and relativistic particles in cluster outskirts. They provide evidence for the acceleration of relativistic particles at shock fronts at large distance (Mpc scale) from the cluster centers (see e.g. Brüggen et al. 2011).

The prototype source of this class is the relic $1253+275$ detected in the Coma cluster (see Giovannini et al. 1991 and references therein), shown in Fig. 10. The Coma relic and most relics show an elongated radio structure, with the major axis roughly perpendicular to the direction of the cluster center. Recently, new spectacular relics have been detected and studied, increasing our knowledge of these diffuse sources. We quote as one of the most interesting cases, the giant narrow relic in CIZA J2242.8+5301 (Sect. 5.1.1). In addition, sources with a more roundish structure, as well as sources of small size, have been detected and classified as cluster relics. Different relic morphologies and related properties will be discussed in the next subsection.

Relics are found in both merging and cool-core clusters, suggesting that they may be related to minor or off-axis mergers, as well as to major mergers. Theoretical models propose that they are tracers of shock waves in merger events. This is consistent with their elongated structure, almost perpendicular to the merger 
axis, and is confirmed by observational results (see e.g. Solovyeva et al. 2008; Finoguenov et al. 2010, and the review by Brüggen et al. 2011).

Currently we know 50 relics hosted in 39 clusters of galaxies: they are reported in Table 3. This collection, which is updated to September, 2011, will be referred to henceforth as September2011-Relic collection. Most clusters are rich Abell clusters, but relics have also been detected in X-ray selected clusters (e.g. RXS J131423.6251521, Valtchanov et al. 2002), and in poor clusters (e.g. S0753, Subrahmanyan et al. 2003).

The classification of relics may be troublesome in some cases. This may cause small differences from other lists in the literature (see e.g. Nuza et al. 2012). In particular, A133 is excluded from the September2011-Relic collection of Table 3 after the discussion given in Randall et al. (2010), who suggest that the source formerly classified as a relic is one of the two lobes of a double radio galaxy, identified with the central cD galaxy. Moreover, we do not consider the relics in $\mathrm{ZwCl} \mathrm{2341.1+0000,}$ since the diffuse radio emission in this cluster is likely originating from a large-scale filament (Giovannini et al. 2010) (see Sect. 8 and Fig. 26), rather than from double relics (van Weeren et al. 2009c).

We also note that diffuse sources which arise from AGN, in which the nuclear activity has ceased, may be sometimes classified as cluster relics. Actually, these sources are not originating from the ICM, and should be more properly considered as dying sources or AGN relics (see Murgia et al. 2011). They will not be considered here because they are not originated by the ICM.

The occurrence of relics in the NVSS sample (Giovannini et al. 1999) is higher in clusters showing high X-ray luminosity, reaching about $25 \%$ in clusters with $L_{x}>$ $5 \times 10^{44} \mathrm{erg} \mathrm{s}^{-1}$ (Giovannini and Feretti 2002). The redshift distribution of clusters of galaxies, hosting at least one relic, is given in Fig. 11, where different colors refer to different structures (Sect. 5.1). As discussed by Giovannini and Feretti (2004), relic sources at very low redshift could have a large angular extension, and therefore may be missed in interferometric observations because of the poor coverage of short spacings. However, owing to the irregular and elongated structure of most relics, this observational problem is not as crucial as in the more regular and diffuse halo sources. Relics are detected up to $z \sim 0.4$, and their distribution with redshift is similar to that of radio halos. The low number of high redshift relics could be due to selection effects, but could also be a real trend, due e.g. to the evolution of cluster mergers, or to the inverse Compton losses which become more relevant at increasing redshift. Deeper radio surveys and cluster catalogues are necessary to investigate this point.

\subsection{Structure}

After the discovery of the prototypical relic $1253+275$ in the Coma cluster (Fig. 10), different morphologies have been found to be associated with relics (see e.g. Giovannini and Feretti 2004): classical sources are elongated and located in the periphery of galaxy clusters, but more regular and roundish structures have also been detected, which are located nearer the cluster center and/or in peripheral regions.

Kempner et al. (2004), in a classification of cluster radio sources, suggested that radio relics could be distinguished in two classes: those associated with a previous 


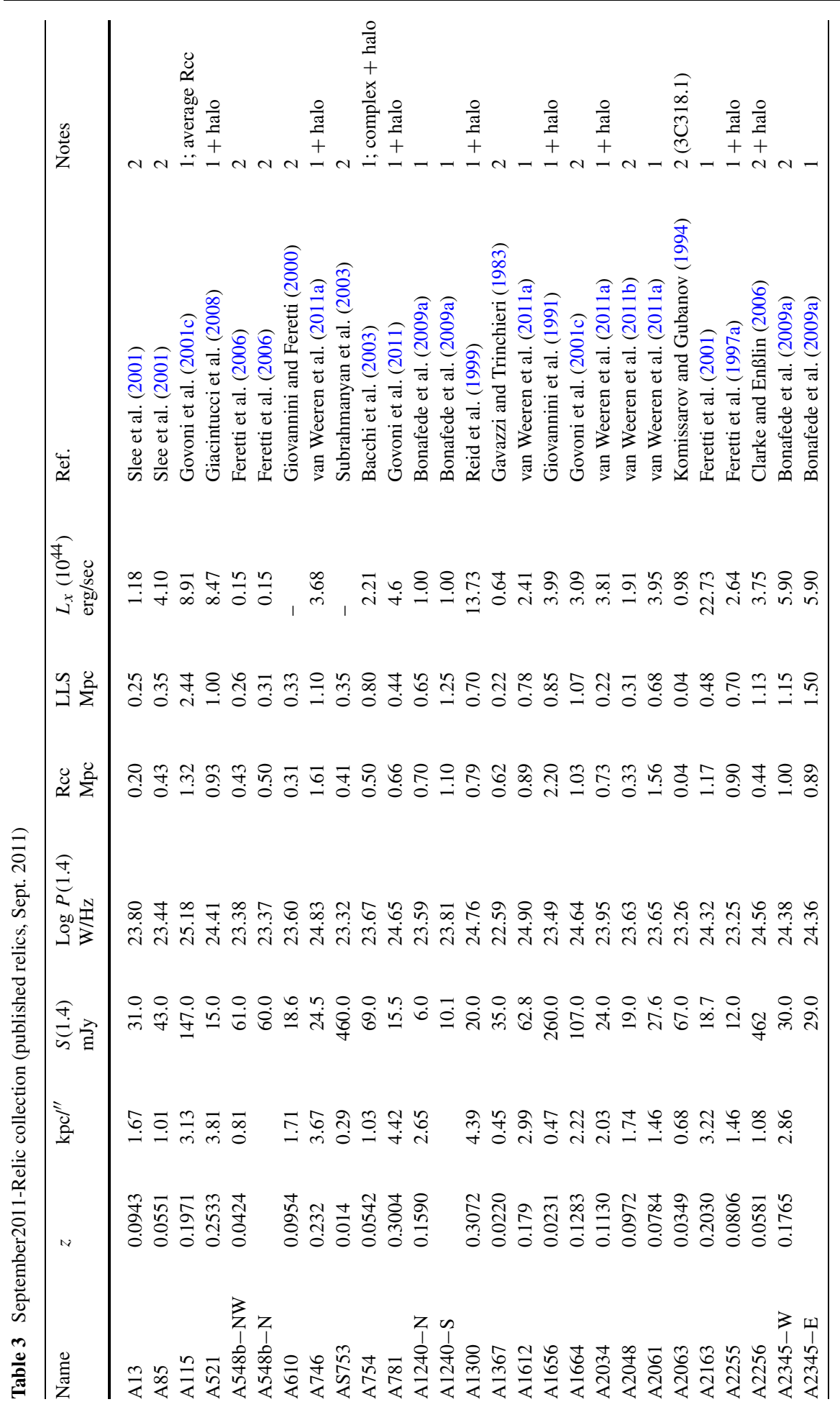




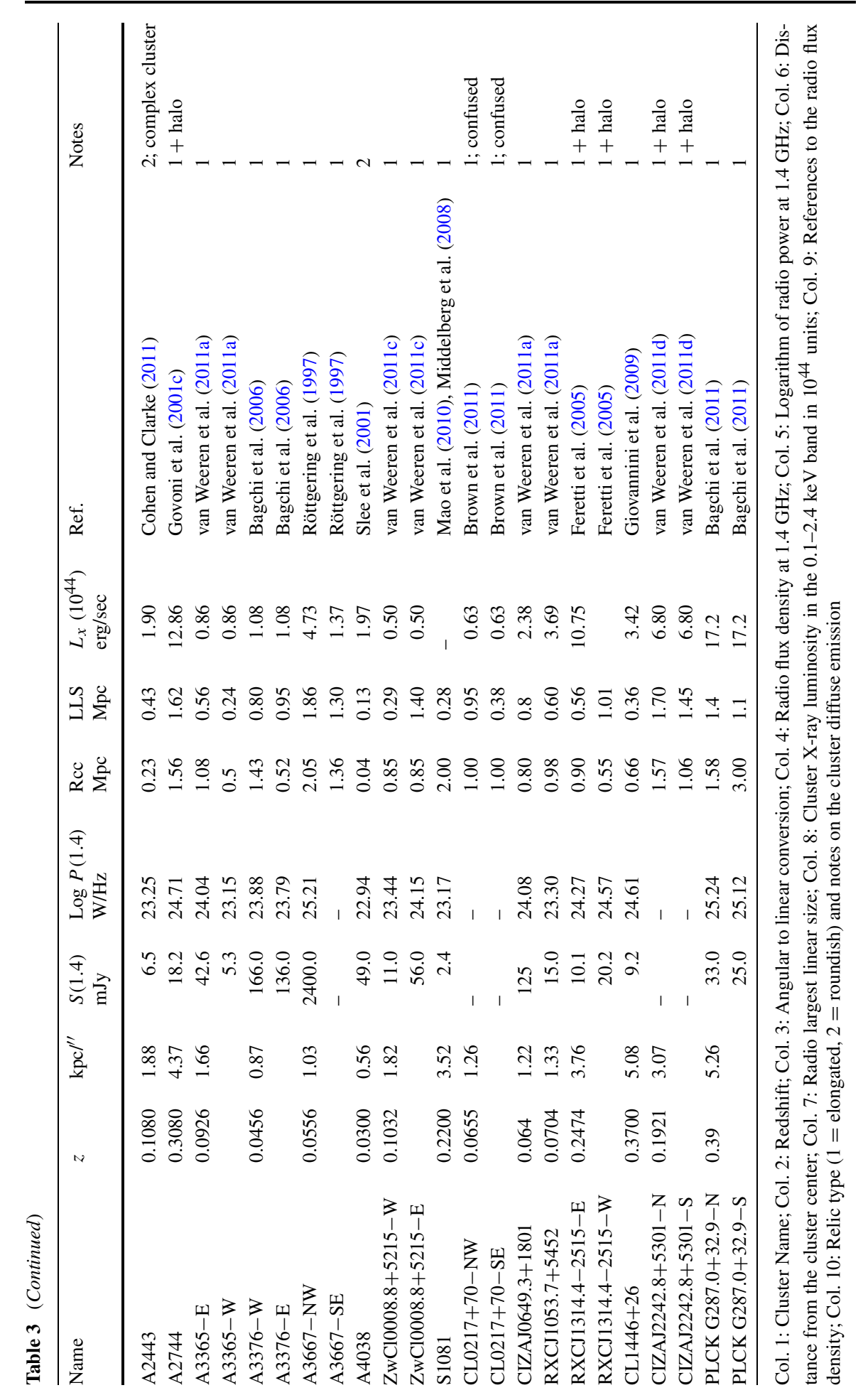


Fig. 11 Distribution of known clusters with radio relics from Table 3 as a function of the cluster redshift. For the classification of elongated (red) and roundish (green) sources see Sects. 5.1.1 and 5.1.2, respectively

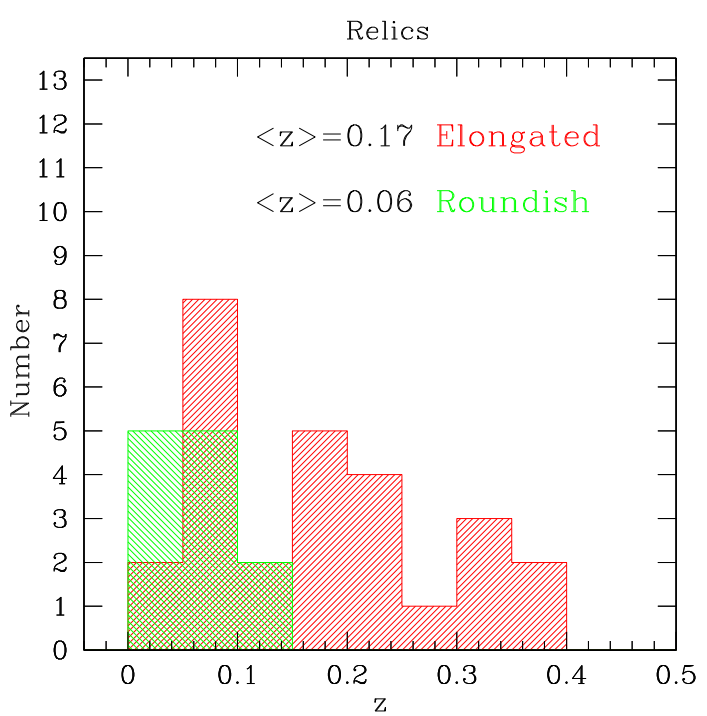

AGN activity, called radio Phoenix, and those related to the ICM, named radio Gischt. This classification has been used somewhat in the literature. However, it implies that the origin of a relic source has to be established, in order to properly classify it.

To be conservative, we prefer to rely on a classification that is based only on the morphology, which is an observable. Therefore we divide relics in elongated and roundish, and obtain the properties of these two classes, to find out whether they may be indications of different origin and physical parameters. In all the following plots, as done in Fig. 11, we will use different symbols for the elongated and roundish relics.

\subsubsection{Elongated relics}

Elongated relics are the classical extended objects, located in cluster peripheral regions, characterized by an elongated shape roughly perpendicular to the cluster center direction, as the relic $1253+275$ in the Coma cluster (Giovannini et al. 1991; Brown and Rudnick 2011; Fig. 10). They do not show any evident substructures, and in some case their transverse size is very small. When observed with high angular resolution, they show an asymmetric transverse profile, with a sharp edge usually on the side toward the cluster outer edge and the radio emission is usually highly polarized. A spectacular example, detected very recently, is represented by the northern relic in CIZAJ2242.8+5301 (van Weeren et al. 2010). This giant relic is very narrow and slightly curved following the cluster boundary. It is strongly polarized, with highly regular polarization vectors, and shows steep spectrum (see Fig. 12). To the south of the cluster, at about $2.8 \mathrm{Mpc}$ distance, a second (fainter) relic structure is present.

We note that projection effects may be important in these elongated structures. An extreme, and unique, case in the double cluster A115 (Govoni et al. 2001c), where the relic apparently starts at the center of the northern cluster and is elongated towards the cluster periphery (Fig. 13, left panel). 

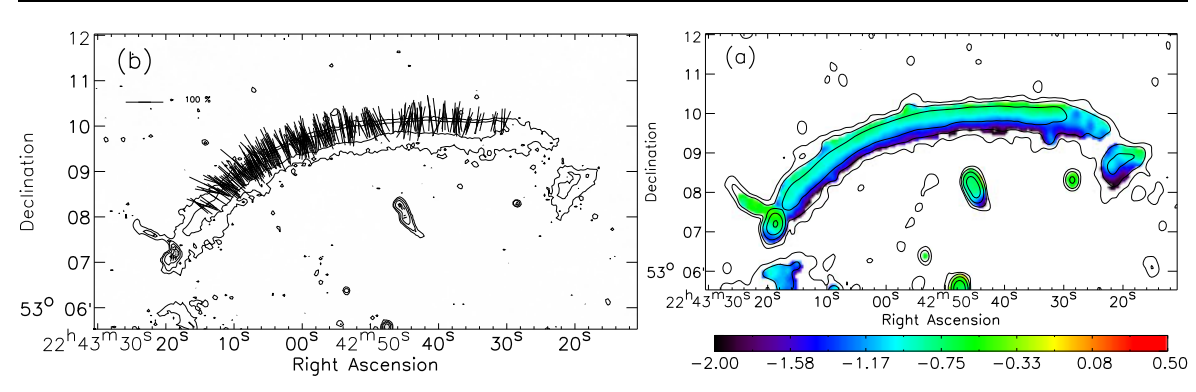

Fig. 12 Left panel: Contours of the radio emission of the northern relic in CIZAJ2242.8+5301, obtained with the GMRT at $610 \mathrm{MHz}$. Superimposed lines represent the polarization electric field vectors obtained with the VLA at $4.9 \mathrm{GHz}$. The length of the vectors are proportional in length to the polarization fraction. Right panel: Radio spectral index map of the same relic, obtained with a power-law fit to measurements at 5 frequencies between 2.3 and $0.61 \mathrm{GHz}$. Contours of the radio emission are from WSRT $1.4 \mathrm{GHz}$. Images are from (van Weeren et al. 2010), to whom we refer for details

These morphologies are in very good agreement with models predicting that these sources are related to large-scale shocks generated during cluster merger events. Indeed, these shocks expanding with high velocity (Mach number $\sim 1-3$ ) can accelerate electrons to high energies, and compress magnetic fields, giving rise to large regions emitting synchrotron radiation. The accelerated particles will have a power-law energy distribution, and magnetic fields aligned parallel to the shock front. This is also consistent with the observed spectral index (see Sect. 5.3 and Fig. 12, right panel) and polarization properties, and explain the shape and location of these sources (see e.g. van Weeren et al. 2011a, and references therein).

\subsubsection{Roundish relics}

In addition to elongated relics, diffuse extended radio sources with a more regular and roundish structure have been detected off-center in clusters: an example is in A1664 (Govoni et al. 2001c), shown in the right panel of Fig. 13. Despite their different structure, these relics share with the elongated relics the location at the cluster periphery and the lack of an optical identification. The main properties of roundish relics are the diffuse morphology resolved in filamentary substructures and a very steep curved spectrum (see e.g. Slee et al. 2001).

The detection of roundish relics might lead to the obvious interpretation that these sources would be elongated relics seen face-on. This is, however, very unlikely, indeed the number of relics with elongated shape is too high to be consistent with simple projection effects. Moreover, we will show in the following and in the next subsections that the properties of elongated and roundish relics may be different.

The class of roundish relics defined here includes the sources named Radio Phoenix by Kempner et al. (2004), or relic Sources near the First Ranked Galaxy (FRG) by Giovannini and Feretti (2004). These objects are located near the central FRG, usually a cD galaxy, but not coincident with it. Their size is in a large range of values, i.e. from $\lesssim 100 \mathrm{kpc}$ (as in A2063 and A4038) to 350 kpc (as in A85). These sources, when observed at higher resolution, show evident filamentary substructures (e.g. Govoni et al. 2001c). Owing to the proximity of these sources to an AGN, an 

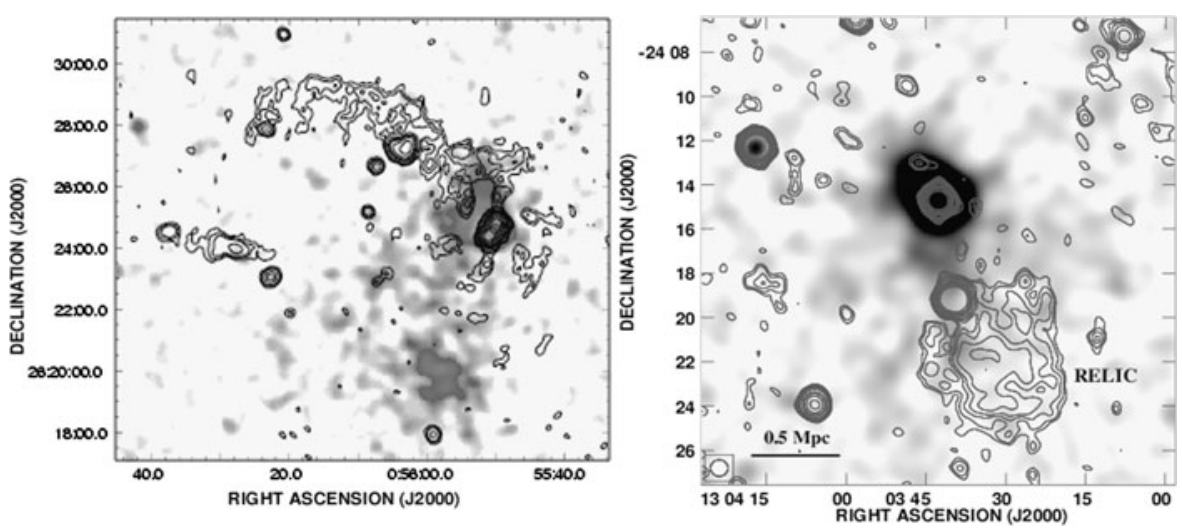

Fig. 13 Radio relics in the clusters: Left panel: A115 $(z=0.197)$, extended from the cluster center toward the cluster periphery, probably because of projection effects: Right panel: A1664 $(z=0.128)$, exhibiting a diffuse roundish structure. In both panels the radio emission at $20 \mathrm{~cm}$ obtained with the VLA is in contours, while the superimposed gray-scale refers to the X-ray emission detected from ROSAT PSPC (see Govoni et al. 2001c)

obvious interpretation is that these sources consist of old radio lobes originated by previous AGN activity, which have become no longer visible because of strong radiation losses, and have been revived by the energy supplied by shock waves. A caveat to this interpretation is that these relics are always located only on one side with respect to the FRG. We note, however, the case of A133, which was classified in the past as a relic (radio Phoenix) but has been recently shown to be an old radio galaxy (Randall et al. 2010). Another possible explanation of the nature of these sources has been supplied by Mathews and Brighenti (2008), who, in their study of the connection between X-ray cavities and radio lobes, suggest that radio bubbles, blown into the cluster gas by a radio galaxy will rise buoyantly and may eventually expand to the cluster outskirts, where the cosmic rays would impact into the surrounding medium, giving rise to small relics located near the AGN.

Among relics with a roundish shape, there are also some objects which are located at large distance from the FRG or any bright galaxy, therefore difficult to reconcile with any model involving a previous radio activity: we already noted A1664 shown in the right panel of Fig. 13; another case is in A548b, where two roundish relics are present, on the same side of the cluster (Feretti et al. 2006; Solovyeva et al. 2008) (see Fig. 1).

More data are necessary to understand the origin and nature of these objects. Interestingly, we note that roundish relics are peaked at lower redshift with respect to the total relic redshift distribution and that no roundish relics at $z>0.2$ are known (Fig. 11).

\subsubsection{Clusters with double relics}

In the framework that relics are witnessing the presence of shock waves originated by mergers between clusters with approximately equal masses and low impact parameter, it is expected that relics should often come in pairs and be located on opposite 


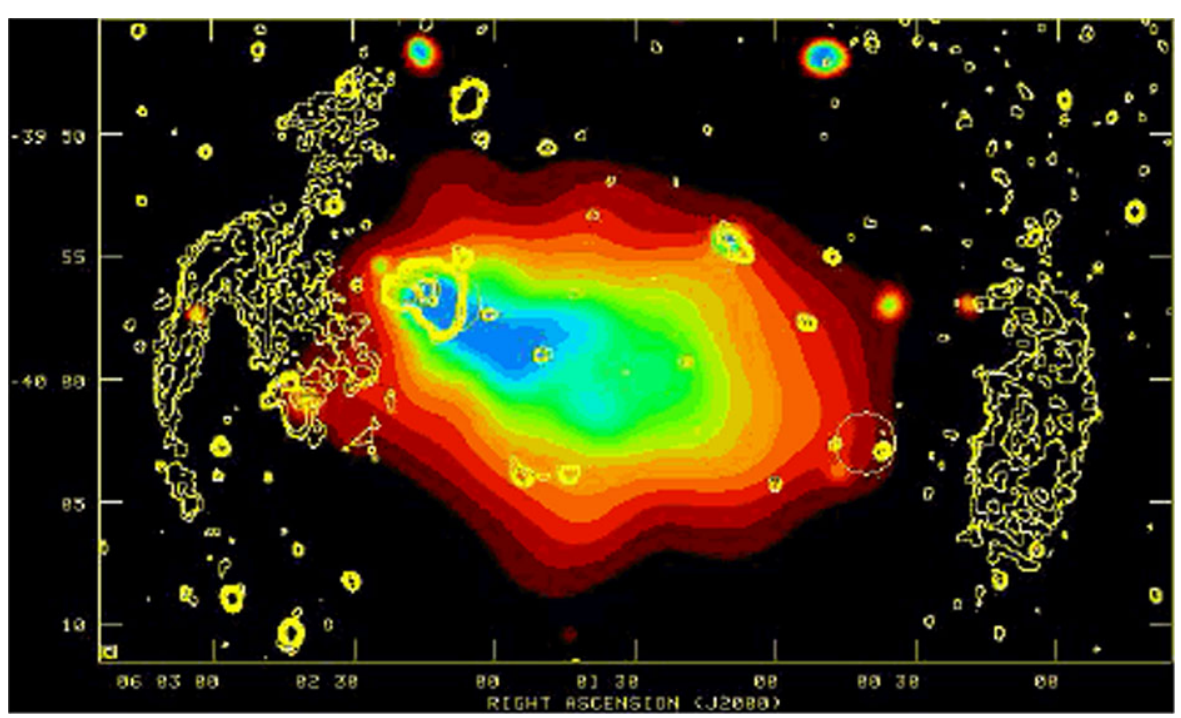

Fig. 14 Double radio relics in the cluster A3376: The radio emission is represented by yellow contours $\left(0.12,0.24,0.48,1 \mathrm{mJy}\right.$ beam $\left.^{-1}\right)$ obtained from VLA observations at $1.4 \mathrm{GHz}$ at the resolution of $20^{\prime \prime}$. The color image depicts the X-ray emission detected by ROSAT PSPC within 0.14 to $2.0 \mathrm{keV}$ band (from Bagchi et al. 2006)

sides of the cluster along the axis merger, with the extended radio structures elongated perpendicularly to this axis (e.g. Röttiger et al. 1999b). The first cluster where two almost symmetric relics have been detected to be located on opposite sides with respect to the cluster center is A3667 (Röttgering et al. 1997; Johnston-Hollitt et al. 2002; Johnston-Hollitt 2003). Several other objects have been found recently, so these cases are getting more and more common, see e.g. A 3376 (Fig. 14, Bagchi et al. 2006), RXCJ1314.4-2515 (Feretti et al. 2005), A 1240 and A 2345 (Bonafede et al. 2009a), CIZAJ2242.8+5301 (van Weeren et al. 2010), ZwCl 0008.8+5215 (van Weeren et al. 2011c), PLCK G287.0+32.9 (Bagchi et al. 2011) and CL0217+70 (see Fig.15 and Brown et al. 2011).

In the case of CIZAJ2242.8+5301, Van Weeren et al. (2011d) discuss, from hydrodynamical simulations and comparison with the data, that the relic morphology arises naturally from shocks produced by a head-on merger in the plane of the sky of two roughly equal mass clusters. In the clusters A1240 and A2345, optical data support the outgoing merger shocks models (Barrena et al. 2009; Boschin et al. 2010). In the case of A3376 (Fig. 14), Bagchi et al. (2006) suggested the possibility that the two relics may be tracing shocks induced by the accretion flows of the intergalactic medium during the large-scale structure formation (accretion shocks Miniati 2003; Keshet et al. 2003). This interpretation is under debate for this cluster (e.g. Akamatsu et al. 2011a), however, it may be invoked to explain the origin of other sources as e.g. the outermost relics in A2255 (Pizzo et al. 2008) and possibly the peripheral emission of the Coma cluster (Brown and Rudnick 2011). 


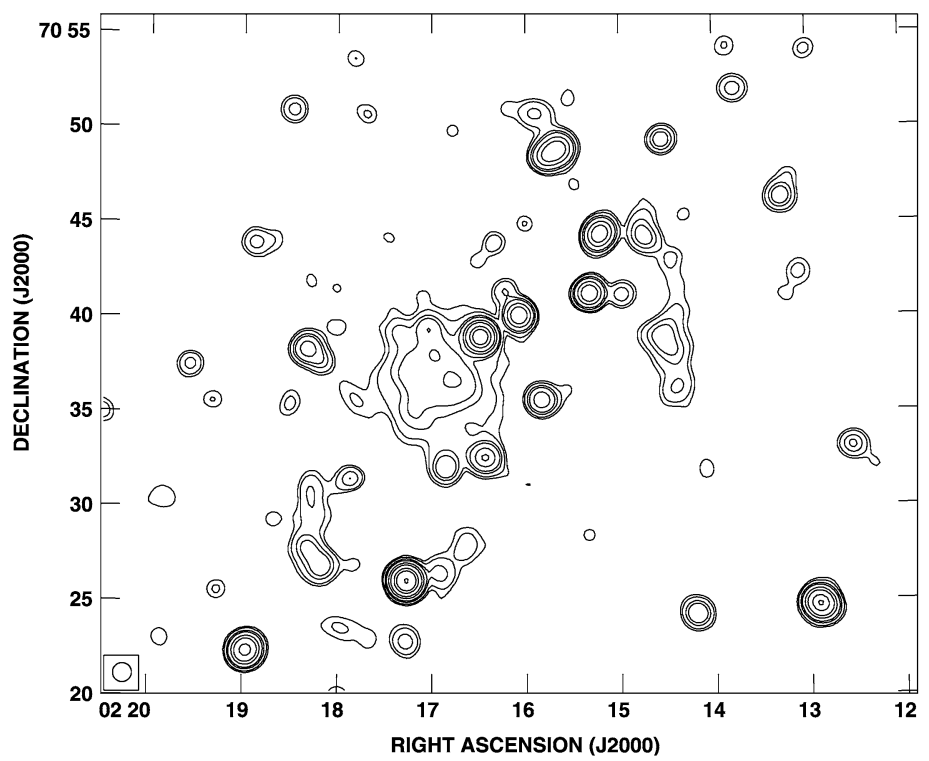

Fig. 15 Radio halo plus double radio relics in the cluster CL0217+70. The HPBW is 60" , and levels are: $0.3,0.5,1,1.5,3,5,10,15,30 \mathrm{mJy} \mathrm{beam}^{-1}$. For more details see Brown et al. (2011)

\subsubsection{Clusters with halo and relic(s)}

As the Coma cluster, several other clusters have been found to show a peripheral relic radio emission in addition to a central halo. According to the September2011Relic collection, at present 11 clusters are known to show this feature. Sometimes, the relic is connected to the radio halo through a low brightness bridge of radio emission (e.g. Coma cluster, A2255 and A2744), other cases are characterized by complex structures, as in A754 (Fig. 1) and A2256. In a few case, clusters may host a central radio halo and double elongated relics. At present we know three clusters showing these features: CL0217+70 (Fig. 15), RXCJ1314.4-2515 (Fig. 1), and CIZAJ2242.8+5301.

These structures are the most extreme examples of the connection between halo sources and cluster mergers (see Sect. 4.3.2). Indeed the merger that gives rise to the radio halo, could also be the origin of shock waves which supply the energy to the peripheral relics. In this scenario, the origin of the bridge of radio emission connecting the halo to the relic source in some clusters has still to be clarified.

We note that in most clusters showing at least a relic source, no central halo is present. This could suggest that relics could be created also by minor mergers, which are not strong enough to produce a radio halo at the cluster center. This is in agreement with the presence of relics in a few cool-core clusters, since a minor and offcenter merger could create a peripheral shock wave, without destroying the central cool-core. As discussed by van Weeren et al. (2011d), double relics should be located in merging systems with a small mass ratio, while single relics should be found in mergers with larger mass ratios. 

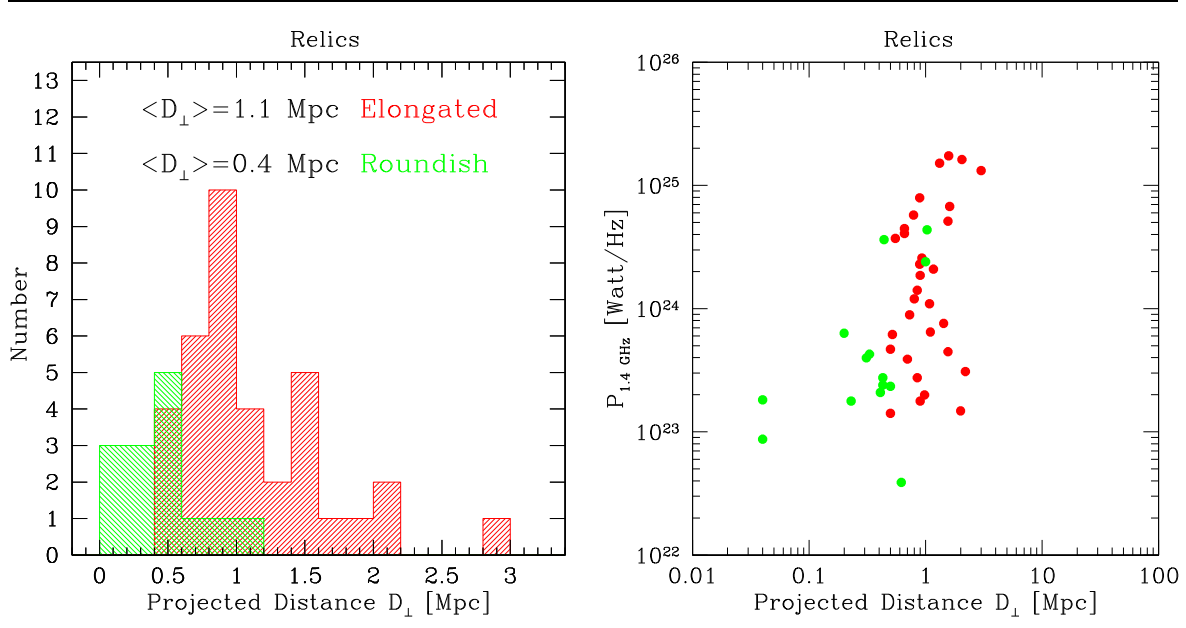

Fig. 16 Left panel: Distribution of relics according to their projected distance from the cluster center. Right panel: Monochromatic radio power of relics at $1.4 \mathrm{GHz}$ versus their projected distance from the cluster center. In both panels, red color refers to the elongated relics, green color to the roundish relics

\subsection{Radio power, size and projected distance}

To better understand the properties of relics, we derived correlations between their observational radio properties and their location in the cluster, using the data of the September2011-relic collection. Double relics in the same clusters are included separately. In the plots, we use different symbols for the elongated and roundish relics, and find that in general elongated and roundish relics are rather separated, with the exception of a few sources.

In the left panel of Fig. 16, the distribution of projected distance of relics from the cluster center is presented. We note that roundish sources are more concentrated toward the cluster center, as expected since this class includes the relics located near the FRG (see Sect. 5.1.2). Their distribution is different from that of elongated relics, with an average projected distance from the cluster center $R_{\mathrm{cc}} \sim 0.4 \mathrm{Mpc}$ suggesting that most of them are really at a small distance from the center. Elongated relics are mostly located between 0.5 and $1.5 \mathrm{Mpc}$ from the center, up to a distance of $3 \mathrm{Mpc}$. They avoid cluster centers and show a distribution peaked at the cluster periphery. From the figure it can be deduced that projection effects are unlikely to play a crucial role, i.e. elongated relics are actually located in the cluster outskirts. This may be due to the fact that high Mach number shocks needed to supply energy to the radio emitting particles are only present in cluster peripheral regions (Gabici and Blasi 2003). This point has been recently discussed in detail by Vazza et al. (2012).

The monochromatic radio power of relics as a function of the projected distance from the cluster center is given in the right panel of Fig. 16. No clear correlation is present. Roundish relics are less powerful than elongated relics, except for a few cases. It is derived that relics, both elongated and roundish, located at distances $\gtrsim 1 \mathrm{Mpc}$ from the cluster center, show powers in the range from $10^{23}$ to $10^{25} \mathrm{~W} \mathrm{~Hz}^{-1}$, whereas there is a lack of powerful $\left(>10^{24} \mathrm{~W} \mathrm{~Hz}^{-1}\right)$ relics near the cluster center 

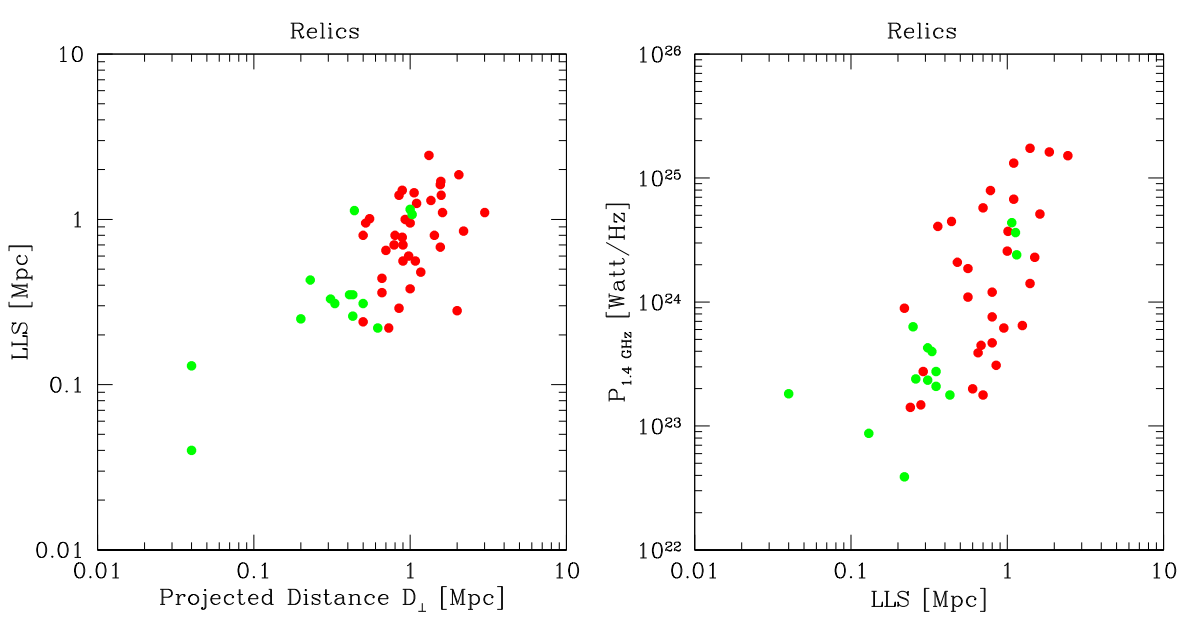

Fig. 17 Left panel: Relic largest linear size (LLS) versus the projected distance from the cluster center; Right panel: Monochromatic radio power of relics at $1.4 \mathrm{GHz}$ versus the relic largest linear size. In both panels, red color refers to the elongated relics, green color to the roundish relics

$(<0.4 \mathrm{Mpc})$, which could be related to the different relic morphology and to the low efficiency of shocks in cluster central regions (see e.g. Vazza et al. 2012).

The left panel of Fig. 17 gives the largest linear size of relics as a function of their projected distance from the cluster center (see also van Weeren et al. 2009b). As in the plot of monochromatic radio power versus projected distance (Fig. 16, right panel), elongated and roundish relics are well separated, except for a few roundish objects, the largest ones, which are in the same region of the plot as the elongated relics.

The monochromatic radio power of relics at $1.4 \mathrm{GHz}$ versus the relic largest linear size (LLS) is presented in the right panel of Fig. 17. Elongated relics show a similar behavior to the roundish relics, although they are somewhat separated on the plot, being on average more powerful and more extended. In particular, relics with low radio powers $\left(<10^{24} \mathrm{~W} \mathrm{~Hz}^{-1}\right)$ can be either roundish or elongated, but the latter have larger size. The most powerful relics are mostly large elongated relics. By considering elongated and roundish relics together, a marginal correlation could be present between LLS and projected distance from the cluster center, in the sense that larger relics are at larger distance from the cluster center.

The summary of the above plots is as follows:

(1) Roundish relics are more often located near the cluster center, while elongated relics are more distributed in peripheral regions.

(2) The properties of roundish and elongated relics are generally rather different. While roundish relics are on average of low power and small size, elongated relics cover a large interval of radio powers and sizes, and are on average more powerful and more extended. Only a few roundish relics show sizes and radio powers similar to those of elongated relics: these objects are the relics in A1664, A2256 and A2345 (western). 
(3) In central cluster regions $(<0.4 \mathrm{Mpc})$, high power $\left(>10^{24} \mathrm{~W} \mathrm{~Hz}^{-1}\right)$ relics are missing. In peripheral regions, on the contrary, there are both small and large size relics, as well as low and high power objects. In general relic properties are in agreement with current models, where shocks are more efficient in low density peripheral regions, where they would give rise to larger and more powerful relics.

\subsection{Radio spectra of relics}

Spectral data for relics are available for several objects. Indeed, the elongated structure of most relic favors their detection with interferometric observations, moreover their position at the cluster periphery, where the number of confusing sources is lower, allows more easily observations at different frequencies. In Table 4 we present the available spectral index information for the objects of the September2011-relic collection. For homogeneity with radio halos, we report an average spectral index value in the range $\sim 327-1415 \mathrm{MHz}$, and refer to single papers on the cluster of galaxies hosting a relic (see Table 3 ) for more detailed information. The note in the last column of the table indicates the spectral shape, in the case that flux density measurements are available at more than two frequencies.

\subsubsection{Integrated spectrum}

Similarly to radio halos, relics show quite steep spectra. This is evident from the spectral index distribution presented in the left panel of Fig. 18. In this plot, as in the following plots, the lower limit for A548b-B $(\alpha>2)$ is not shown. Elongated relics show spectral index $\alpha$ in the range 1-1.6, with an average value of 1.3. Spectral indices of roundish relics are in a much larger range of values: $\alpha=1.1-2.9$, with an average value of 2.0. Therefore, there is a remarkable difference in spectral index properties between elongated and roundish relics, indeed spectra of roundish relics are steeper than those of elongated relics, with very few exceptions. It is interesting to note that among the roundish relics with $\alpha<1.6$, there are the three objects, whose properties have been outlined in Sect. 5.2, characterized by power, size and distance from the cluster center similar to those of elongated relics, i.e. A $1664(\alpha=1.1)$, A 2256 $(\alpha=1.2)$, and A $2345 \mathrm{~W}(\alpha=1.5)$. For objects with measurements at more than two frequencies, the radio spectrum is straight for elongated relics, while it shows a high frequency steepening for roundish relics.

In the right panel of Fig. 18, we show the relic spectral index versus the relic projected distance from the cluster center. We note that relics with steepest spectra are at small distances from the cluster center. From the plots of the spectral index versus the relic power, and of the spectral index versus the LLS, presented in the 2 panels of Fig. 19, it is derived that most powerful $\left(P_{1.4}>10^{24} \mathrm{~W} \mathrm{~Hz}^{-1}\right)$ and most extended (LLS $>0.5 \mathrm{Mpc}$ ) relics are characterized by less steep spectra $(\alpha<1.6)$. Smaller/low power relics have a spectral index in the range $\sim 1.0-2.8$ (see also van Weeren et al. 2009b).

These results are in agreement with the expected origin of all relic sources from strong shock waves: in peripheral regions they should have a larger Mach number and therefore they should produce flatter spectra (see e.g. van Weeren et al. 2009b). 
Table 4 Spectral index of radio relics

Col. 1: Cluster name;

Col. 2: Total spectral index value; Col. 3: Relic type; Col. 4: Note on the spectral shape

\begin{tabular}{|c|c|c|c|}
\hline Name & $\alpha$ & Type & Notes \\
\hline A13 & 2.3 & Roundish & Steepening \\
\hline A85 & 2.9 & Roundish & Steepening \\
\hline A115 & 1.2 & Elongated & \\
\hline A521 & 1.5 & Elongated & Straight \\
\hline $\mathrm{A} 548 \mathrm{~b}-\mathrm{A}$ & 2.0 & Roundish & \\
\hline $\mathrm{A} 548 \mathrm{~b}-\mathrm{B}$ & $>2$ & Roundish & \\
\hline A610 & 1.4 & Roundish & \\
\hline AS753 & 2.1 & Roundish & Steepening \\
\hline A754 & 1.5 & Elongated & Complex \\
\hline A781 & 1.2 & Elongated & \\
\hline A1240N & 1.2 & Elongated & \\
\hline A1240S & 1.3 & Elongated & \\
\hline A1300 & 1.1 & Elongated & \\
\hline A1367 & 1.9 & Roundish & \\
\hline A1612 & 1.4 & Elongated & Straight \\
\hline A1664 & 1.1 & Roundish & \\
\hline A1656 & 1.2 & Elongated & Straight \\
\hline A2048 & 1.7 & Roundish & Steepening \\
\hline A2061 & 1.0 & Elongated & \\
\hline A2063 & 2.9 & Roundish & Steepening \\
\hline A2163 & 1.0 & Elongated & \\
\hline A2255 & 1.4 & Elongated & \\
\hline A2256 & 1.2 & Roundish & Complex \\
\hline A2345W & 1.5 & Roundish & \\
\hline A2345E & 1.3 & Elongated & \\
\hline A2443 & 2.8 & Roundish & Steepening \\
\hline A2744 & 1.1 & Elongated & \\
\hline A3667NW & 1.1 & Elongated & Straight \\
\hline A4038 & 2.2 & Roundish & Steepening \\
\hline ZwCl0008-W & 1.5 & Elongated & Straight \\
\hline ZwCl0008-E & 1.6 & Elongated & Straight \\
\hline CL0217+70-NW & 1.4 & Elongated & \\
\hline CL0217+70-SE & 1.5 & Elongated & \\
\hline RXCJ1314-E & 1.2 & Elongated & \\
\hline RXCJ1314-W & 1.4 & Elongated & \\
\hline CIZAJ2242-N & 1.1 & Elongated & Straight \\
\hline PLCK G287.0+32.9-N & 1.3 & Elongated & \\
\hline PLCK G287.0+32.9-S & 1.5 & Elongated & \\
\hline
\end{tabular}



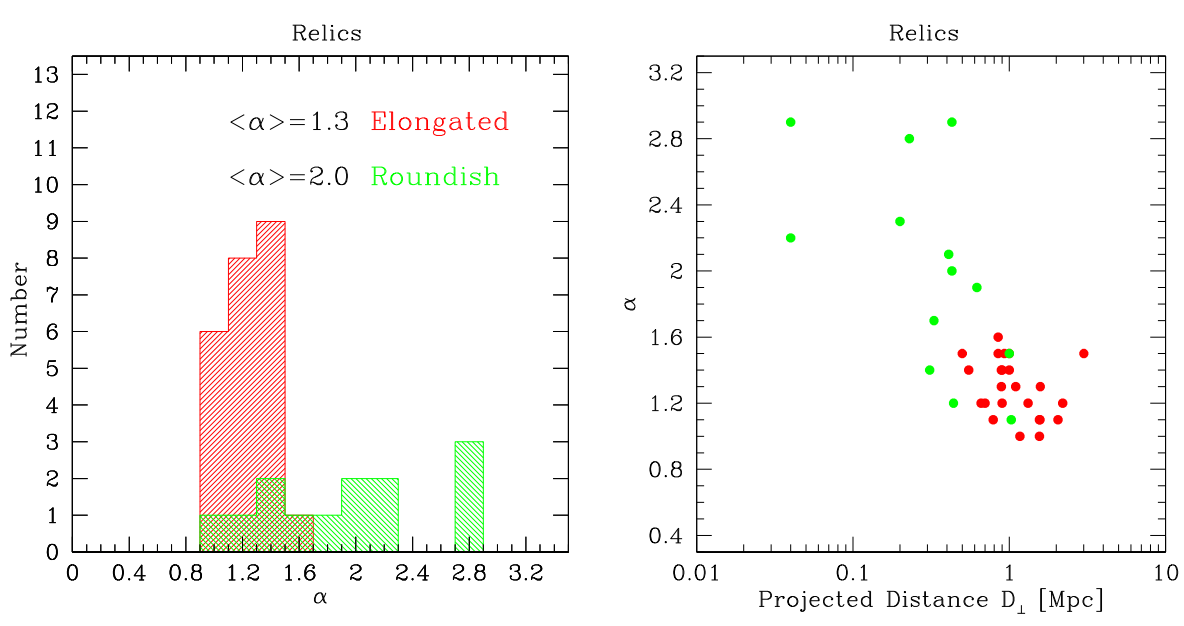

Fig. 18 Left panel: Spectral index distribution of relics. Right panel: Plot of the relic spectral index as a function of the relic projected distance from the cluster center. In both panels, red color refers to the elongated relics, green color to the roundish relics. In these plots, the lower limit $\alpha>2$ for A 548b-B is not shown

\subsubsection{Spectral index distribution}

Spectral index maps have been obtained so far only for a few relics. Elongated relics show a well defined spectral index distribution, with a transverse steepening toward the cluster center. This steepening is clearly detected e.g. in A 2744 (Orrù et al. 2007) (Fig. 6, left panel), and in CIZAJ2242.8+5301 (van Weeren et al. 2011d) (Fig. 12, right panel). This trend is in agreement with the relic brightness profile and strongly supports the shock model (see e.g. Brüggen et al. 2011; van Weeren et al. 2011d for a recent discussion).

Among roundish sources, a spectral index map has been obtained for A 2256 (Clarke and Enßlin 2006), which shows spectral steepening from NW to SE. In other clusters, available images of the spectral index do not show any obvious distribution. The radio morphology at high resolution is filamentary and the spectral index distribution is irregular.

\subsubsection{Ultra-steep spectrum relics}

As outlined in Sect. 5.3.1, roundish relics may exhibit extremely steep spectra ( $\alpha \gtrsim 2$ ). Examples of these sources are in A13, A85, A4038 (see Slee et al. 2001), and A2443 (see Cohen and Clarke 2011). The extremely steep spectra also show a high frequency cutoff. These objects have different properties from the giant elongated relics, being on average less powerful and less extended. The number of these sources is very small. A reason could be that they are the tip of the iceberg of a population of ultra-steep relics that may be detected at low frequency, similarly to the ultra-steep halos illustrated in Sect. 4.1.3. Alternatively, we could also consider possible selection effects: indeed small-size relics which are marginally resolved may be often classified as background discrete radio galaxies. 

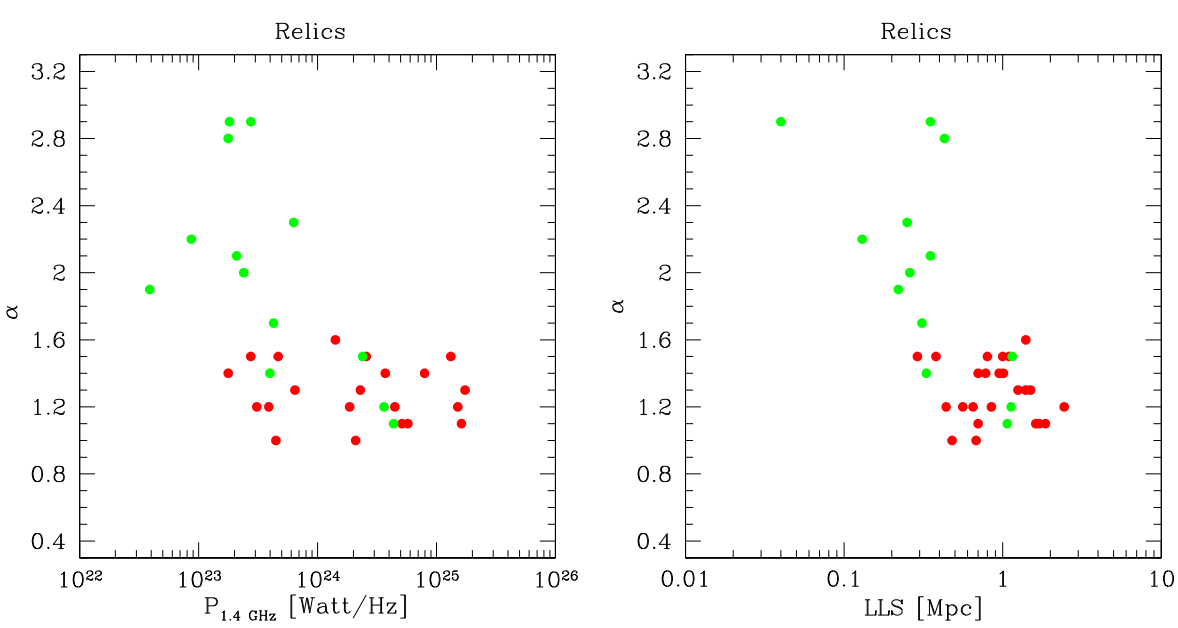

Fig. 19 Left panel: Relic spectral index as a function of the monochromatic radio power at $1.4 \mathrm{GHz}$; Right panel: Relic spectral index versus the largest linear size (LLS). In both panels, red color refers to the elongated relics, green color to the roundish relics. In these plots, the lower limit $\alpha>2$ for A 548b-B is not shown

Fig. 20 Monochromatic radio power of relics at $1.4 \mathrm{GHz}$ versus the cluster X-ray luminosity between $0.1-2.4 \mathrm{keV}$. Red color refers to the elongated relics, green color to the roundish relics

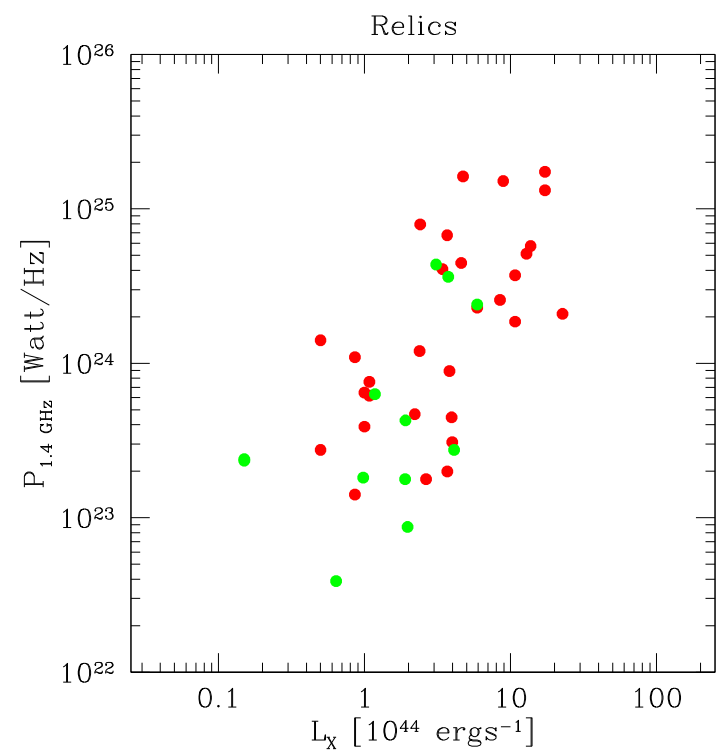

We can argue that the nature of roundish, ultra-steep relics is different from that of other relics, either elongated or roundish, and that the spectral index is likely a key parameter for the classification of relics.

\subsection{Radio-X-ray connection and comparison with halos}

The properties of relics have been found to be related to the properties of their parent clusters. Indeed, similarly to radio halos, a correlation is found between the relic radio 

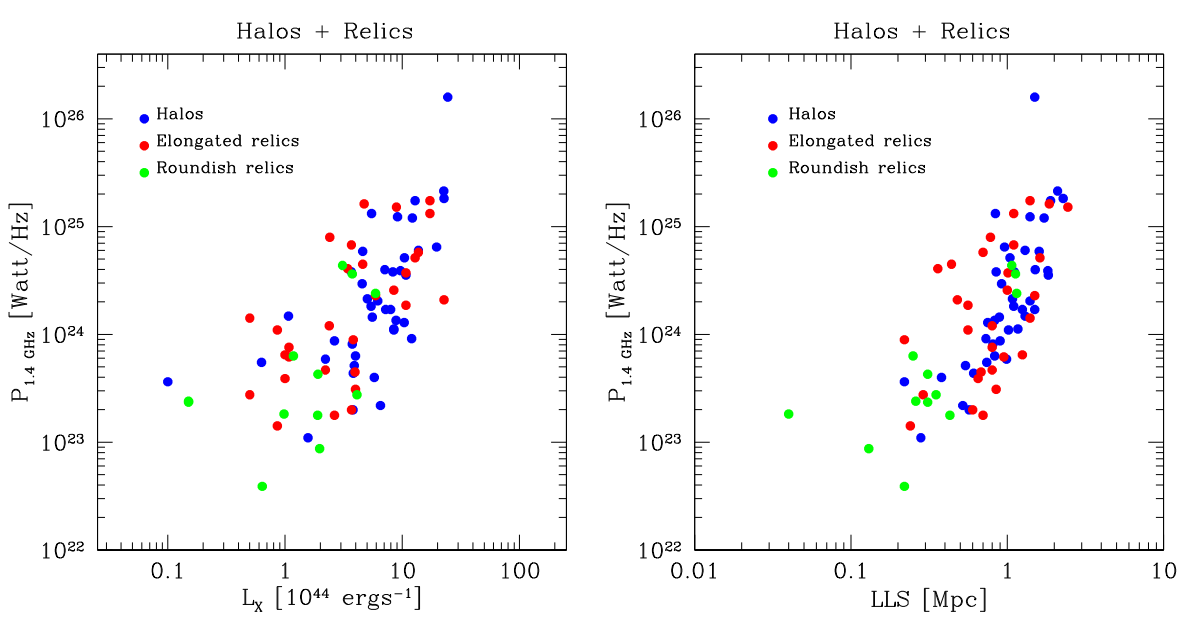

Fig. 21 Left panel Monochromatic radio power of halos and relics at $1.4 \mathrm{GHz}$ versus the cluster X-ray luminosity. Right panel: Monochromatic radio power of halos and relics at $1.4 \mathrm{GHz}$ versus their largest linear size measured at the same frequency. In both panels, blue dots represent halos, while red and green dots represent elongated and roundish relics, respectively

power at $1.4 \mathrm{GHz}$ and the X-ray luminosity of clusters (Fig. 20). The correlation holds for both elongated and roundish relics, although with somewhat large dispersion; no systematic difference is visible between the two classes of relics. We find $P_{1.4} \propto L_{x}^{1.2}$.

The similarity between the properties of halos and relics has been already noted in the past (e.g. Giovannini and Feretti 2002). In Fig. 21 we show the halo and relic radio power as a function of the X-ray cluster luminosity (left panel), and as a function of their linear size (right panel). For clarity, we use different symbols for halos, elongated relics and roundish relics. The most powerful object in the plots is the radio halo in the cluster MACS J0717.5+3745.

It turns out that halos and relics show a very similar behavior in both plots, supporting the connection between halos and relics, and their link to cluster mergers. From the left panel of Fig. 21, we note that the most powerful halos and relics show similar values in radio power and cluster X-ray luminosity (with the exception of MACS J0717.5+3745), with similar dispersion. At lower radio powers, there is a large dispersion in both classes, and in particular it is interesting to note that, while halos in poor X-ray clusters are only a few (Sect. 4.3.1), relics are easily detected also in clusters of low X-ray luminosity. Indeed, elongated and roundish relics fill the region of radio power $P_{1.4}<10^{24} \mathrm{~W} \mathrm{~Hz}^{-1}$ and cluster X-ray luminosity $L_{x}<3 \times 10^{44}$. The flatter correlation found for relics with respect to halos likely reflects this behavior.

Halos and relics show similar behavior also in the plot of radio power versus largest linear size (right panel of Fig. 21). As for the previous correlation, halos show a lower dispersion in the data, and at a given radio power they tend to be more extended than relics. We finally note the peculiarity of MACS J0717.5+3745 (Bonafede et al. 2009b; van Weeren et al. 2009a; see Sects. 4.2 and 4.3), which, even with the addition of relics, remains the most powerful diffuse radio source, with a radio power about an order of magnitude higher than that of other known diffuse sources. 


\section{Mini-halos}

Some relaxed, cool-core, galaxy clusters exhibit signs of diffuse synchrotron radio emission that extend far from the dominant radio galaxy at the cluster center, forming a so-called mini-halo. Mini-halos are extended on a moderate scale ( $\simeq 500 \mathrm{kpc})$ and in common with halos and relics they have a low surface brightness and a steep spectrum. The diffuse source in the Ophiucus cluster (Govoni et al. 2009; Murgia et al. 2010b), reported in Fig. 22, is an example of a mini-halo.

Mini-halos are sometimes considered small versions of radio halos, but their classification is more complex and fuzzy. Their emission originates from relativistic particles and magnetic fields which are believed to be deeply mixed with the thermal intracluster gas. As a general guideline, this physical characteristic can be used to distinguish mini-halos from other kind of steep spectrum radio sources in clusters such as radio bubbles related to AGN activity. We shall not define as mini-halos those radio sources in which the ambient thermal gas is clearly separated by the non-thermal plasma, as in the case of AGN radio lobes whose expansion has created cavities or holes in the intracluster X-ray emission. An example of this situation is given by the radio source 3C317 at the center of A2052 (e.g. Blanton et al. 2011). 3C 317 is composed of a compact core surrounded by an amorphous halo. This kind of radio morphology is also referred to as a core-halo (see e.g. Mazzotta and Giacintucci 2008). Although the radio emission at the center of A2052 resembles on a smaller scale the typical mini-halo, almost all of the radio emission detected so far is contained within

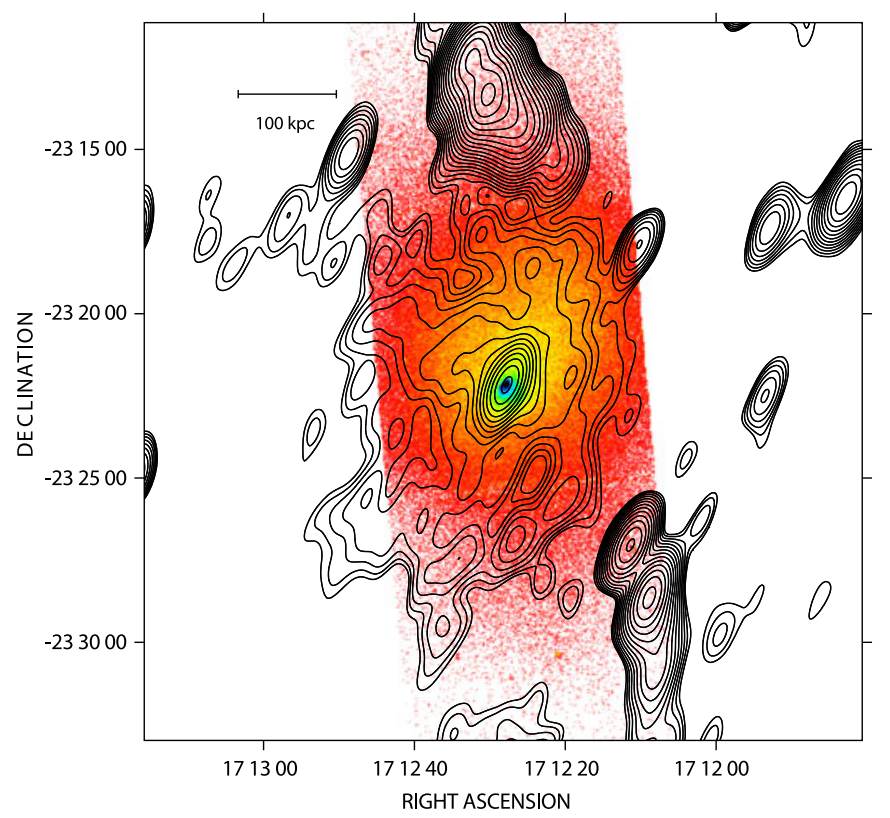

Fig. 22 VLA $1.4 \mathrm{GHz}$ contours of the mini-halo in Ophiuchus overlaid on the Chandra X-ray emission. The resolution of the radio image is $91.4^{\prime \prime} \times 40.4^{\prime \prime}$. The first contour level is drawn at $0.3 \mathrm{mJy} \mathrm{beam}^{-1}$ and the rest are spaced by a factor $\sqrt{2}$. Taken from Govoni et al. (2009) 
cavities devoid of X-ray emitting gas. Therefore, this type of radio sources will not be considered here.

The Perseus cluster is a different case. The radio source $3 \mathrm{C} 84$ at the center of Perseus shows X-ray cavities in the inner region where the AGN radio activity interacts with the thermal gas (e.g. Fabian et al. 2003), but on larger scale the cluster exhibits a diffuse radio emission mixed with the thermal intracluster gas. Indeed Perseus cluster represents the prototype example of a mini-halo (e.g. Noordam and de Bruyn 1982; Pedlar et al. 1990; Burns et al. 1992). When the cluster X-ray information is lacking, as for the diffuse emission found in the system MRC 0116+111 (Bagchi et al. 2009), the mini-halo classification can be particularly troublesome.

In addition to the above mentioned difficulties in the mini-halo classification, the relatively small angular size of mini-halos in combination with a possibly strong emission of the central radio galaxy, complicates their detection. Thus, our current observational knowledge on mini-halos is limited to a few clusters. Continued searches have so far revealed no more than 11 mini-halos. A list of mini-halos is given in Table 5. This collection, which is updated to September, 2011, will be referred to henceforth as September2011-Mini-halo collection. Other mini-halos candidates have been found in A1068, and A1413 (Govoni et al. 2009), although to be classified as minihalos they would require further investigation.

The spectra of mini-halos are steep, as well as for halos, with some evidence of radial spectral steepening (e.g. Perseus (Sijbring 1993; Gitti et al. 2002), A 2626 (Gitti et al. 2004), Ophiucus (Murgia et al. 2010b)).

The origin of mini-halos is still poorly known. Gitti et al. (2002) argued that the radio emitting particles in mini-halos cannot be connected to the central radio galaxy in terms of particle diffusion. They proposed that mini-halos result from a relic population of relativistic electrons reaccelerated by MHD turbulence via Fermi-like processes, the necessary energetics being supplied by the cool-core region. This is supported by the correlation observed between the mini-halo radio power and the cooling rate power (Gitti et al. 2004).

The analysis of the mini-halo present in the most X-ray luminous cluster RXJ1347.5-1145 (Gitti et al. 2007), suggests that additional energy for electron reacceleration in mini-halos might be provided by sub-cluster mergers that have not been able to destroy the central cluster cool-core. In this cluster, the spatial correspondence between a radio excess of the mini-halo emission and a hot region, detected through both Sunyaev-Zel'dovich effect (see Fig. 23) and X-ray observations, indicates that electron reacceleration in this location is most likely related to a shock front propagating into the intracluster medium (Ferrari et al. 2011). In addition to RXJ1347.5-1145, other clusters of galaxies like Ophiuchus (Govoni et al. 2009) and A2142 (Giovannini and Feretti 2000) present a radio mini-halo, as well as a cool-core that has likely survived a possible recent merging event. Indeed, although cool-core clusters are generally considered relaxed systems, when analyzed in detail they sometimes reveal peculiar X-ray features in the cluster center which may be indicative of a link between the mini-halo emission and some minor merger activity.

Finally, cold fronts associated to the core gas sloshing have been observed in some clusters hosting a mini-halo. Thus, the possibility has been advanced that gas sloshing may generate turbulence in the core, which in turn may reaccelerate the relativistic electrons necessary to form a mini-halo (ZuHone et al. 2011). 
Table 5 September2011-Mini-halos collection (published mini-halos, September 2011)

\begin{tabular}{|c|c|c|c|c|c|c|}
\hline Cluster & $z$ & $\mathrm{kpc} /{ }^{\prime \prime}$ & $\begin{array}{l}S(1.4) \\
\mathrm{mJy}\end{array}$ & $\begin{array}{l}\log P(1.4) \\
\mathrm{WHz}^{-1}\end{array}$ & $\begin{array}{l}L_{x}[0.1-2.4 \mathrm{keV}] \\
10^{44} \mathrm{erg} / \mathrm{sec}\end{array}$ & Ref. \\
\hline Perseus & 0.0179 & 0.36 & 3020.0 & 24.33 & 7.88 & Sijbring (1993) \\
\hline Ophiuchus & 0.028 & 0.55 & 106.4 & 23.27 & 6.11 & $\begin{array}{l}\text { Govoni et al. } \\
\text { (2009) }\end{array}$ \\
\hline A1835 & 0.2532 & 3.91 & 6.0 & 24.06 & 24.15 & $\begin{array}{l}\text { Govoni et al. } \\
\text { (2009) }\end{array}$ \\
\hline A2029 & 0.0765 & 1.43 & 18.8 & 23.42 & 9.24 & $\begin{array}{l}\text { Govoni et al. } \\
(2009)\end{array}$ \\
\hline A 2142 & 0.0894 & 1.65 & 18.3 & 23.55 & 11.47 & $\begin{array}{l}\text { Giovannini and } \\
\text { Feretti }(2000)\end{array}$ \\
\hline A2390 & 0.228 & 3.62 & 63.0 & 24.98 & 5.33 & $\begin{array}{l}\text { Bacchi et al. } \\
(2003)\end{array}$ \\
\hline A2626 & 0.0604 & 1.15 & 29.0 & 23.39 & 1.18 & Gitti et al. (2004) \\
\hline MRC0116+111 & 0.131 & 2.31 & 140.0 & 24.79 & - & $\begin{array}{l}\text { Bagchi et al. } \\
(2009)\end{array}$ \\
\hline RBS 797 & 0.35 & 4.91 & - & - & 16.91 & Gitti et al. (2006) \\
\hline RXJ1347.5-1145 & 0.451 & 5.74 & 25.2 & 25.27 & 51.95 & Gitti et al. (2007) \\
\hline RXCJ1504.1-0248 & 0.2153 & 3.46 & 20.0 & 24.42 & 27.55 & $\begin{array}{l}\text { Giacintucci et al. } \\
\text { (2011b) }\end{array}$ \\
\hline
\end{tabular}

Col. 1: Cluster name; Col. 2: Redshift; Col. 3: Angular to linear size conversion; Col. 4: Radio flux density at $1.4 \mathrm{GHz}$; Col. 5: Logarithm of radio power at $1.4 \mathrm{GHz}$; Col. 6: Cluster X-ray luminosity in the 0.1$2.4 \mathrm{keV}$ band in $10^{44}$ units; Col. 7 : References to the radio flux density

\subsection{Comparison with halos}

Cassano et al. (2008) found that the synchrotron emissivity of mini-halos is about a factor of 50 higher than that of radio halos. In the framework of the particle reacceleration scenario, they suggested that an extra amount of relativistic electrons would be necessary to explain the higher radio emissivity of mini-halos. These electrons could be provided by the central radio galaxy (e.g. Fujita et al. 2007) or be of secondary origin as previously suggested in the literature by some authors (e.g. Pfrommer and Enßlin 2004; Keshet and Loeb 2010). In this latter case relativistic electrons in minihalos are expected to be continuously produced by the interaction of cosmic-ray protons with the ambient thermal protons.

Murgia et al. (2009) modeled the radio brightness profile $I(r)$ of radio halos and mini-halos, with an exponential of the form $I(r)=I_{0} e^{-r / r_{e}}$. The proposed method to derive the central radio brightness, the length-scale, and hence the radio emissivity of diffuse sources is relatively independent of the sensitivity of the radio observation. Figure 24 shows the best fit central brightness $I_{0}$ versus the length-scale $r_{e}$ for a representative set of radio halos and mini-halos. These authors found that radio halos can have quite different length-scales but their emissivity is remarkably similar from one halo to another. In contrast, mini-halos span a wide range of radio emissivity. Some of them, like Perseus, RXJ1347.5-1145 and A2390 are characterized 


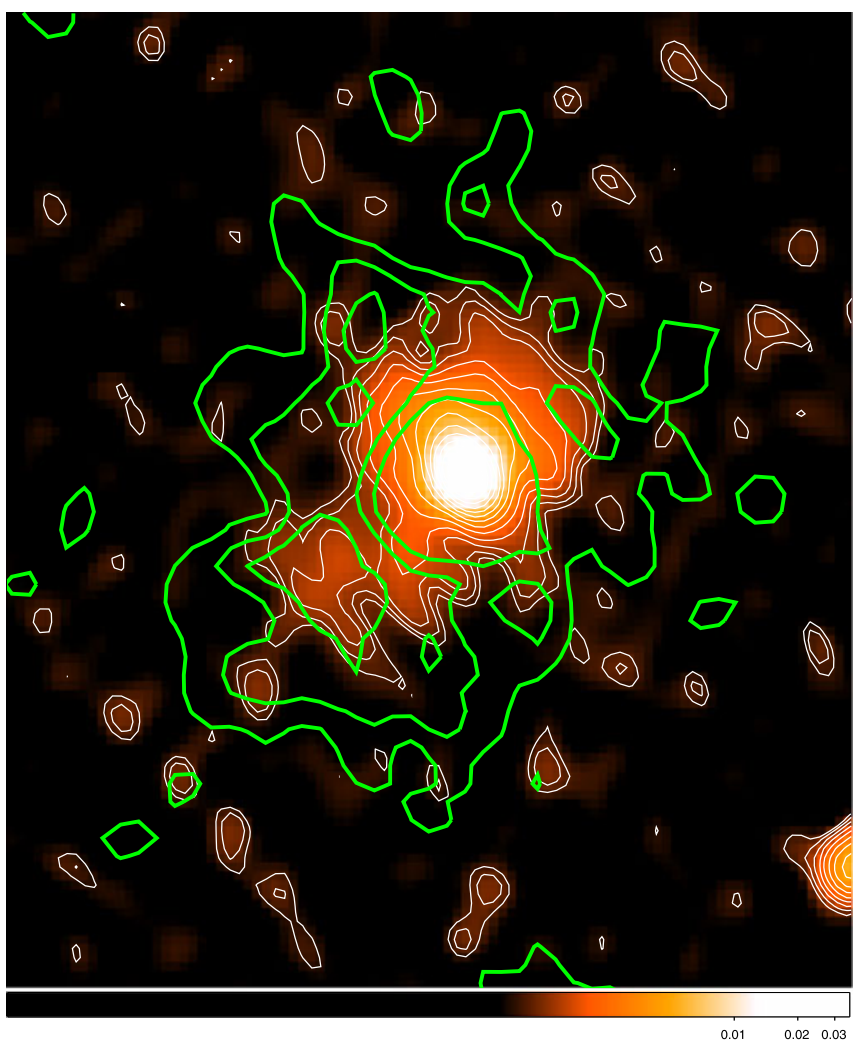

Fig. 23 GMRT $614 \mathrm{MHz}$ total intensity map and contours (white) of the mini-halo in RXJ1347.5-1145. The resolution is $4.8^{\prime \prime} \times 3.5^{\prime \prime}$. The first contour levels are drawn at $-0.3,0.3 \mathrm{mJy}$ beam $^{-1}$ and the rest are spaced by a factor $\sqrt{2}$. Contours of the MUSTANG SZE image (Mason et al. 2010) of the cluster are overlaid in green. Image taken from Ferrari et al. (2011)

by a radio emissivity which is more than two order of magnitude greater than that of radio halos. This finding seems to suggest that part of the mini-halo radio emission is related to properties of the local intergalactic medium and part is correlated with the AGN activity of the central brightest galaxy, as suggested by the faint correlation discussed by Govoni et al. (2009), between the mini-halo and the $\mathrm{cD}$ radio power. On the other hand there are also mini-halos like A2029, Ophiuchus, and A1835 that have a radio emissivity which is much more typical of halos in merging clusters rather than of the other mini-halos.

\section{Magnetic fields and observational results}

Thanks to new radio observations and to the improvement of interpretative models, our understanding of magnetic fields associated with the intracluster medium in clusters of galaxies has advanced significantly in recent years and the presence of $\mu \mathrm{G}$-level magnetic fields in clusters of galaxies is now widely acknowledged. 


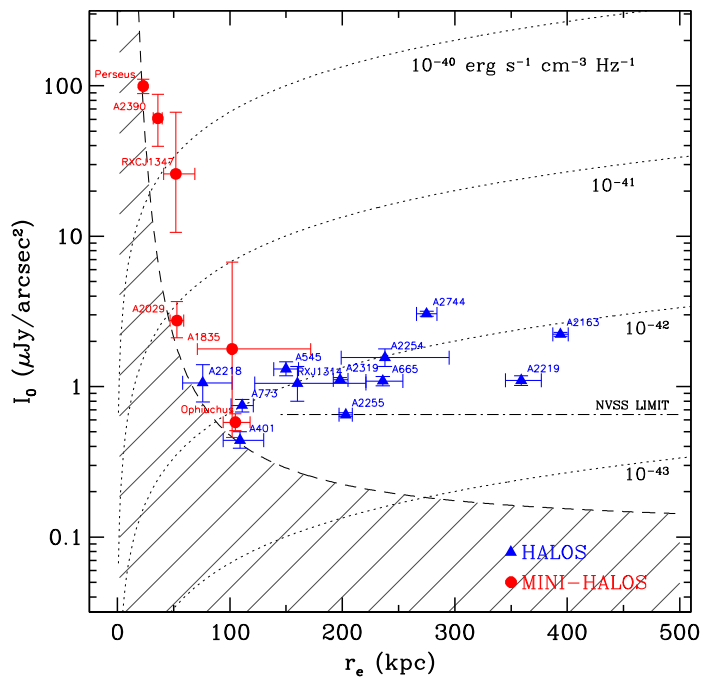

Fig. 24 Best fit central brightness $I_{0}$ versus the length-scale $r_{e}$ both for halos (blue triangles) and mini-halos (red dots) taken from Murgia et al. (2009). The dotted lines indicate regions of constant emissivity, namely $0.1,1,10,100$ times the average emissivity of radio halos which is $\langle J\rangle \simeq 10^{-42} \mathrm{erg} \mathrm{s}^{-1} \mathrm{~cm}^{-3} \mathrm{~Hz}^{-1}$. They have been traced by assuming a putative redshift of $z=0.18$ and a spectral index $\alpha=1$. The dashed line represents the detection limit expected for a mini-halo or a halo at $z=0.18$ observed in a deep image with a beam of $25^{\prime \prime}$ and a sensitivity level of $25 \mu \mathrm{Jy} \mathrm{beam}^{-1}$. The dot-dashed line represents the $3 \sigma$ sensitivity level of the NVSS

Most of what we know about intracluster magnetic fields derives from the study of cluster diffuse radio sources (halos, relics and mini-halos) and Faraday Rotation Measures of polarized radio galaxies located inside or behind galaxy clusters. One of the first comprehensive reviews on extragalactic magnetic field was presented by Kronberg (1994), followed by more recent works (Carilli and Taylor 2002; Govoni and Feretti 2004).

Large-scale magnetic fields are studied through radio halos, which reveal magnetic fields spread over extremely large volumes. Sometimes, the intracluster magnetic fields can be ordered on scales of hundreds of kpc, as revealed in A2255 (Govoni et al. 2005; Pizzo et al. 2011), and MACS J0717.5+3745 (Bonafede et al. 2009b), where a polarized signal has been detected. The brightness fluctuations and polarization level of radio halos are strictly related to the intracluster magnetic field structure. For example, lack of polarization and a smooth and regular surface brightness may indicate that the cluster magnetic field is ordered on small scales, while a disturbed radio morphology and presence of polarization could be related to a magnetic field ordered on large scales (Tribble 1991a; Murgia et al. 2004). Currently, only in the radio halos of A 2255 and A 665, brightness fluctuations and polarization information have been used to put some constraints on the magnetic field power spectrum of a galaxy cluster (Govoni et al. 2006; Vacca et al. 2010).

The most promising technique to derive a detailed view of the intracluster magnetic fields on small spatial scales is the analysis of the Faraday rotation of radio 
galaxies located inside and beyond clusters. The Faraday effect is the rotation suffered by linearly polarized radiation traveling through a magnetized plasma. The intrinsic polarization angle $\psi_{0}$ is rotated by an amount:

$$
\Delta \psi=\psi-\psi_{0}=\lambda^{2} R M
$$

where $\lambda$ is the radiation wavelength while $R M$ is the so-called Rotation Measure. The rotation measure is defined by

$$
R M=812 \int_{0}^{L} n_{e} B_{z} d l\left(\operatorname{rad~m}^{-2}\right)
$$

where $n_{e}$ is the thermal electron density in $\mathrm{cm}^{-3}, B_{z}$ is the magnetic field component along the line-of-sight in $\mu \mathrm{G}$, and $L$ is the path length through the plasma in kpc (Burn 1966). If we perform multi-frequency observations of a radio source embedded in or behind a galaxy cluster, then by a linear fit of the $\lambda^{2}$-law in Eq. (1) we can estimate the $\mathrm{RM}$ along that direction. By knowing the RM and given a model for the distribution of the thermal electrons (e.g. from X-ray observations) we can infer the intracluster magnetic fields. However, because of the random and turbulent nature of intracluster magnetic fields, inverting Eq. (2) is not straightforward even in the cases of simple electron density distributions.

A technique to analyze and interpret the RM data is the RM Synthesis (Brentjens and de Bruyn 2005), which uses the RM transfer function to solve the $n \pi$ ambiguity related to the RM computation, and allows distinguishing the emission as a function of Faraday depth.

Detailed RM images of extended radio sources have been obtained (e.g. Perley and Taylor 1991; Taylor and Perley 1993; Feretti et al. 1995, 1999a; Feretti 1999; Govoni et al. 2001a; Taylor et al. 2001, 2007; Eilek and Owen 2002; Govoni et al. 2006; Guidetti et al. 2008, 2010; Laing et al. 2008; Bonafede et al. 2010; Govoni et al. 2010). The RM distributions seen across these sources present patchy structures whose statistics is, in first approximation, Gaussian-like with dispersions up to several hundred (and even thousand) $\mathrm{rad} \mathrm{m}^{-2}$. The observed RM fluctuations indicate that the intracluster magnetic field is not regularly ordered but turbulent on scales ranging from tens of $\mathrm{kpc}$ to $\lesssim 100 \mathrm{pc}$.

The early interpretation of this finding was that the intracluster magnetic field is composed by uniform cells of size $\Lambda_{C}$ with random orientation in space. In this "single-scale" model (e.g. Lawler and Dennison 1982; Tribble 1991b; Feretti et al. 1995; Felten 1996), the RM distribution is expected to be a Gaussian with zero mean and dispersion given by

$$
\sigma_{\mathrm{RM}}^{2}=812 \Lambda_{C} \int_{0}^{L}\left(n_{e} B_{z}\right)^{2} d l\left(\operatorname{rad}^{2} \mathrm{~m}^{-4}\right) .
$$

Thus, by measuring the dispersion of the RM we can estimate the cluster magnetic field provided that the cell size $\Lambda_{C}$ is known. The question is then: what exactly is $\Lambda_{C}$ and how can we find it? This issue was investigated theoretically and in numerical tests by different authors (Enßlin and Vogt 2003; Vogt and Enßlin 2003, 2005; Murgia et al. 2004), who determined that the correct value for $\Lambda_{C}$ is the magnetic field autocorrelation length, $\Lambda_{B}$, which can be calculated if the power spectrum of the magnetic field fluctuation is known. Hence, if we want to determine the strength of the magnetic field we ultimately need to determine the power spectrum of its fluctuations. 


\subsection{Faraday rotation modeling}

Murgia et al. (2004) and Laing et al. (2008) developed dedicated software tools to attempt to constraint the magnetic field power spectrum parameters. These codes have been designed to produce, starting from 3D magnetic field models, synthetic polarization images that can be directly compared with the observations. To limit the degrees of freedom and the computational burden, in many of these simulations the magnetic field power spectrum is assumed to be a simple power-law of the form $\left|B_{k}\right|^{2} \propto k^{-n}$. However, in some cases high-order terms in curvature have been also explored (Laing et al. 2008; Guidetti et al. 2010). Another more general method, relying on a semianalytical approach and Bayesian inference has been also developed by Enßlin and Vogt (2003). The power spectrum shape is decomposed with multiple spectral basis components and it is free to assume, in principle, any form required by the data (Enßlin and Vogt 2003; Kuchar and Enßlin 2011).

In all the mentioned modeling, it is assumed that the power spectrum normalization scales as a function of the gas density such that $B(r)=B_{0}\left(\frac{n_{e}(r)}{n_{0}}\right)^{\eta}$, where the index $\eta$ is in the range $0.5-1.0$ (Dolag et al. 2001). Indeed there are several indications that the magnetic field intensity declines with radius with a rough dependence on the thermal gas density. This is predicted by both cosmological simulations (e.g. Dolag et al. 1999; Brüggen et al. 2005; Xu et al. 2010) and by the comparison between thermal and radio brightness profiles (Govoni et al. 2001b) in clusters hosting radio halos.

We note that in agreement with the absence of a preferred direction in most of the RM images, the results of the modeling are based on the assumption that the magnetic field is statistically isotropic and that the Faraday rotation is occurring entirely in the intracluster medium with a negligible local RM enhancement occurring at the interface between the radio lobes and the surrounding medium (but see Rudnick and Blundell 2003 for a contrary viewpoint). However, recent RM images (Guidetti et al. 2011) of four radio galaxies $(0206+35,3$ C270, 3C353, M84) located in environments ranging from poor groups to rich clusters show clearly anisotropic "banded" patterns. In some cases, these RM patterns coexist with regions of isotropic random variations. These RM bands may be interpreted as a consequence of interactions between the sources and their surroundings and are likely be caused by magnetized plasma which has been compressed by the expanding radio lobes.

\subsection{Cluster centers}

The Faraday rotation modeling described above has been applied to interpret the RM images of single extended radio galaxies located at the center of galaxy clusters. Some of them present strong RM asymmetries between the two lobes. This is the LaingGarrington effect: the farthest lobe suffers a higher Faraday rotation due to the largest amount of intervening material (Laing 1988; Garrington et al. 1988; Garrington and Conway 1991). Indeed, at least for these sources, we know that the observed RM must originate mostly in the foreground magnetized intracluster medium.

Radio sources at the center of dense cool-core clusters, like Hydra-A, probe magnetic fields as high as 10-30 $\mu \mathrm{G}$ (Laing et al. 2008; Kuchar and Enßlin 2011). The 
$\mathrm{RM}$ analysis of radio sources in the less dense environments of galaxy groups, such us 3C31 or 3C449 (Feretti et al. 1999b; Laing et al. 2008; Guidetti et al. 2010), indicates lower central magnetic field strengths in the range 3-10 $\mu \mathrm{G}$. The intracluster magnetic field auto-correlation length is generally found to be of the order of a few kpc.

Determining the detailed shape of the magnetic field power spectrum it is not trivial because of the degeneracy existing between its slope and the outer scale of the magnetic field fluctuations, $\Lambda_{\max }$ (Murgia et al. 2004). In fact, the same RM images can be explained either by a flat power spectrum with a large $\Lambda_{\max }$ or by a steeper power spectrum with a lower $\Lambda_{\max }$. Given the limited spatial dynamic range of the current RM images, it is still not easy to break the degeneracy. According to Kuchar and Enßlin (2011), the slope magnetic field power spectrum is close to the Kolmogorov index in Hydra-A while other authors report a flatter slope (Laing et al. 2008).

\subsection{Cluster peripheries}

To get information on the radial trend of the cluster magnetic field, one has to take into account that in the previously described modeling, another important degeneracy exists between the central magnetic field strength $B_{0}$ and the index $\eta$, which describes the scaling of $B$ with the gas density. The same RM image can be explained either by a higher $B_{0}$ with a rapid radial decrease (high $\eta$ ) or by a lower $B_{0}$ with a flatter radial profile. The degeneracy can be solved if RM images of different radio sources at different impact parameters from the cluster center are available.

In A2382 the RM of two tailed sources have been investigated (Guidetti et al. 2008). In this cluster, the power spectrum of the RM fluctuation is compatible with the Kolmogorov index. The observed $\sigma_{\mathrm{RM}}$ decreases in the cluster outskirts and can be explained if $B_{0} \simeq 3.5 \mu \mathrm{G}$ and $\eta=0.5$. But it is also marginally compatible with a flat magnetic field profile with $B_{0} \simeq 0.9 \mu \mathrm{G}$ or with a higher central magnetic field strength of $B_{0} \simeq 13 \mu \mathrm{G}$ which decreases with radius following the thermal gas density, $\eta=1$.

In a few other Abell clusters, the RM of radio sources at different impact parameters have been obtained. In A119 (Feretti et al. 1999a; Murgia et al. 2004), the RM analysis indicates a central magnetic field strength of $B_{0} \simeq 5.5 \mu \mathrm{G}$ which decreases with radius roughly following the gas density. The case of A2255 is particularly interesting since this cluster hosts a Mpc-scale radio halo. By analyzing the RM of three different radio sources and the radio halo properties, a central magnetic field strength of about $2 \mu \mathrm{G}$ for $\eta=0.5$ has been obtained (Govoni et al. 2006). These results would imply that the magnetic field strength in clusters with or without a diffuse halo is indeed very similar.

The RM of radio sources in the Coma cluster, are probably the best studied so far (Kim et al. 1990; Feretti et al. 1995; Bonafede et al. 2010). Bonafede et al. (2010), by comparing the RM observed in seven radio sources (see Fig. 25) with simulations, found a cluster magnetic field in the range $B_{0}=3.9 \mu \mathrm{G} ; \eta=0.4$ and $B_{0}=5.4 \mu \mathrm{G}$; $\eta=0.7$. These values correspond to models where the magnetic field energy density scales as the gas energy density, or the magnetic field is frozen into the gas, respectively. This is expected since the energy in the magnetic component of the intracluster 

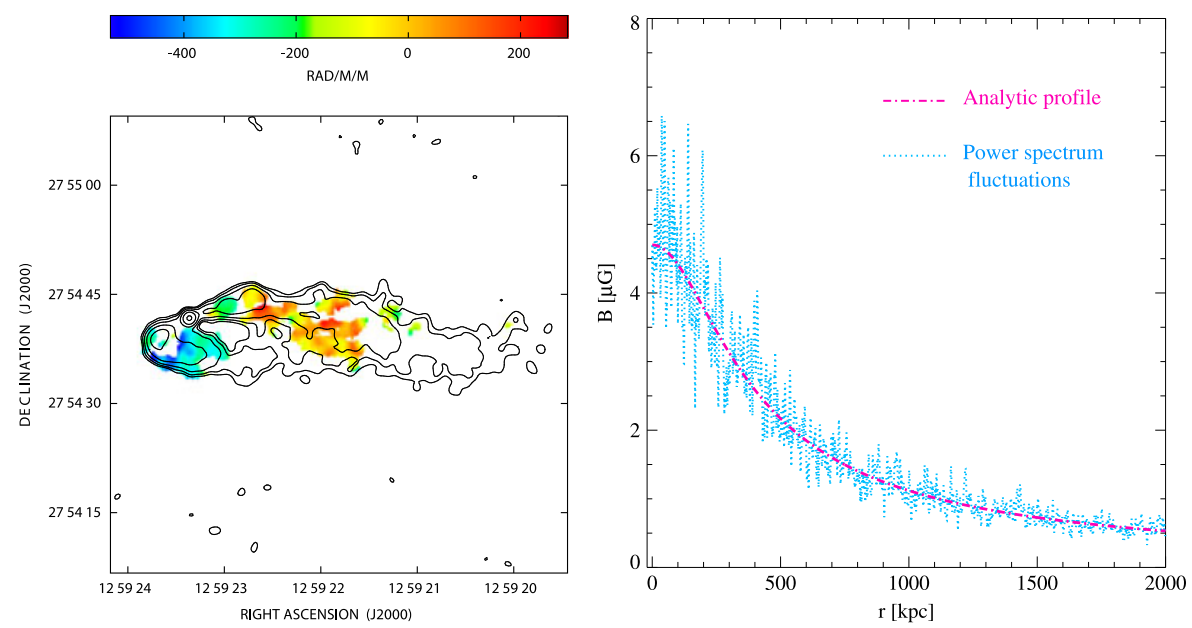

Fig. 25 Left panel: Rotation Measure image (along with the total intensity radio contours at $5 \mathrm{GHz}$ ) of the radiogalaxy NGC 4869. This is one of the seven radio sources used for the analysis of the Coma cluster magnetic field (Bonafede et al. 2010). Right panel: Profile of the best magnetic field model of the Coma cluster (Bonafede et al. 2010). Magenta line refers to the analytic profile $B(r)=B_{0}\left(\frac{n_{e}(r)}{n_{0}}\right)^{\eta}$, while the blue line refers to a slice extracted from the simulated magnetic field numerical model. Power spectrum fluctuations in the profile are shown

medium is a tiny fraction of the thermal energy. It is important to note that the average magnetic field intensity over a volume of $\sim 1 \mathrm{Mpc}^{3}$ is $\sim 2 \mu \mathrm{G}$. Indeed, the model derived from RM analysis gives an average estimate that is compatible with the minimum energy condition in the radio halo (Thierbach et al. 2003). A direct comparison with the magnetic field estimate derived from the inverse Compton emission is more difficult since the hard- $X$ detection is debated. The field strength derived from RM analysis gives a magnetic field estimate that is consistent with the present lower limits obtained from hard X-ray observations (Wik et al. 2009) while it is still a bit higher than the estimate obtained on the basis of the non-thermal hard X-ray detection from RXTE (Rephaeli and Gruber 2002) and BeppoSAX (Fusco-Femiano et al. 2004).

\subsection{Statistical studies}

In addition to detailed RM studies focused on single clusters, interesting analysis have been carried out also by obtaining RM of radio galaxies through a sample of clusters. Clarke et al. (2001) analyzed the average RM values as a function of source impact parameter for a sample of 16 Abell clusters. They found a clear broadening of the RM distribution toward small projected distances from the cluster center clearly indicating, as suggested also by other statistical investigations (e.g. Lawler and Dennison 1982; Vallée et al. 1986; Kim et al. 1991; Johnston-Hollitt et al. 2004), that most of the RM contribution comes from the intracluster medium and proving that magnetic fields are present in all galaxy clusters. A similar correlation has been recently found by Govoni et al. (2010). They analyzed a sample of 12 galaxy clusters in which they included only those clusters hosting 
radio sources for which high-quality, high-resolution, extended RM images are available. Although the statistic is still limited, they found that the RM of radio galaxies in clusters containing a radio halo seem to show a similar behavior to that of clusters without diffuse synchrotron emission.

Increasing attention is given in the literature to the connection between the magnetic field strength and the cluster temperature. These studies are based on cosmological simulations e.g. (Dolag et al. 1999, 2001; Brüggen et al. 2005; Xu et al. 2010) or plasma physical considerations (Kunz et al. 2011). The comparison between clusters with high and low temperatures (Govoni et al. 2010) does not yields significant differences in the RM data, indicating that a possible connection between the magnetic field strength and the gas temperature, if present, is very weak.

Very recently Bonafede et al. (2011) selected a sample of 39 massive galaxy clusters from the HIghest X-ray FLUx Galaxy Cluster Sample (Reiprich and Böhringer 2002), and used NVSS data (Condon et al. 1998) to analyze the trend of the fractional polarization of radio sources lying at different projected distances from the cluster center. They detected a clear trend of the fractional polarization, being smaller for sources close to the cluster center and increasing with increasing distance form the cluster central regions. The higher RM suffered by sources in the inner regions, where both magnetic field strength and gas density are higher, causes in fact a higher beam depolarization. Sources at larger radii, instead, are subject to less depolarization, since their emission is affected by lower RM. Such a trend can be reproduced by a magnetic field model with central value of few $\mu \mathrm{G}$. In addition there seems no differences in the depolarization trend observed in cluster with and without radio halo, indicating that magnetic fields in galaxy clusters are then likely to share the same properties regardless of the presence of radio emission from the intracluster medium.

\section{Beyond clusters}

Cosmological theories and simulations predict that galaxy clusters are connected by intergalactic filaments along which they accreate mass. Shocks from infall into and along the filaments are expected to accelerate particles. These accelerated particles can emit synchrotron radiation if cosmic magnetic fields are present. Attempts to detect diffuse radio emission beyond clusters, i.e. in very rarefied regions of the intergalactic space, have shown recent promise in imaging diffuse synchrotron radiation of very low level.

Bagchi et al. (2002) suggested the presence of radio emission from the filament surrounding the cluster $\mathrm{ZwCl} 2341.1+0000$. This finding has been recently confirmed with deeper observations by Giovannini et al. (2010) (Fig. 26), thus proving that intergalactic filaments can be magnetized. Another filamentary structure extending over 3.3 Mpc is centered on the cD galaxy of the cluster A3444 (Giovannini et al. 2009). The large size of this radio feature, as well as its morphology, rule out a direct connection with the central galaxy and invite comparison with the filamentary structures found in $\mathrm{ZwCl} 2341.1+0000$ (see also van Weeren et al. 2009c). The presence of large-scale radio emission in regions in between galaxy clusters, (e.g. 0917+75; Harris et al. 1993; Giovannini and Feretti 2000), and giant ring-like features (e.g. A3376; 


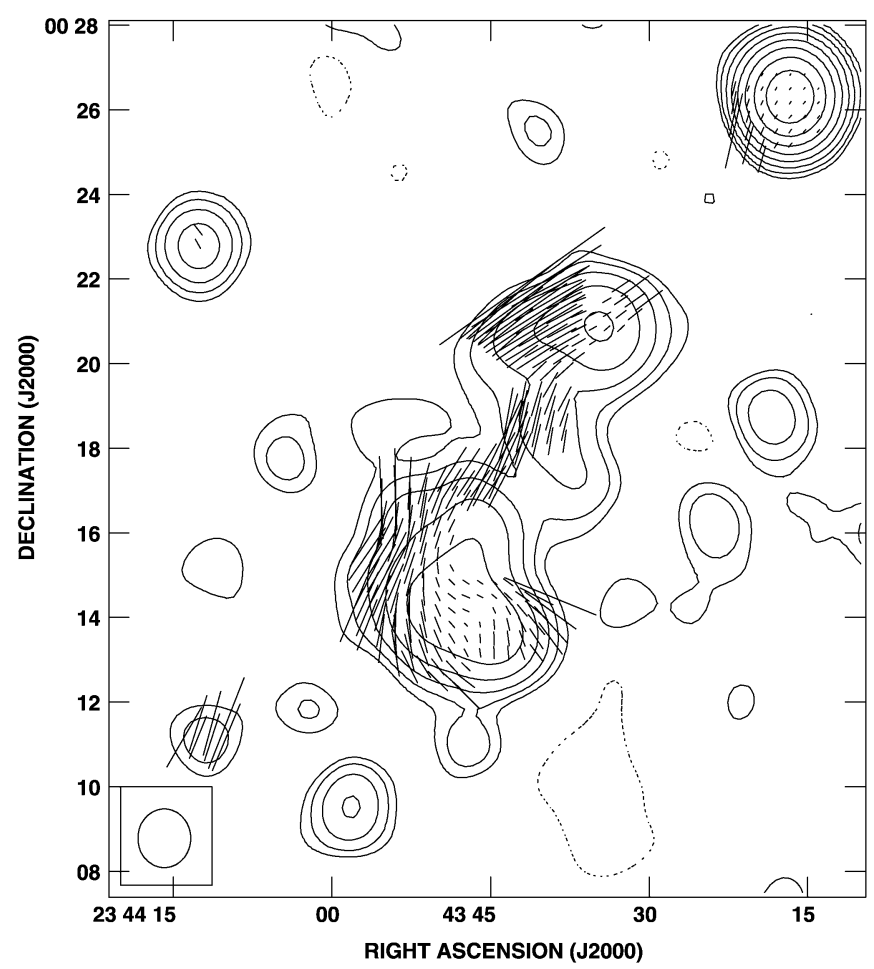

Fig. 26 Large-scale radio emission of the filament $\mathrm{ZwCl} 2341.1+0000$, obtained with the VLA at $1.4 \mathrm{GHz}$ (see Giovannini et al. 2010), after subtraction of unrelated point-sources, at the resolution of $83^{\prime \prime} \times 75^{\prime \prime}(\mathrm{HPBW})$. Contours show the total intensity emission, at levels of $0.15 \mathrm{mJy}$ beam $^{-1}$ and following contours spaced by a factor of 2 . Lines refer to the polarized emission: the orientation represents the projected E-field (not corrected for galactic rotation), the length is proportional to the fractional polarization ( $1^{\prime \prime}$ corresponds to $0.2 \%$ )

Bagchi et al. 2006) seems to be related to the large-scale structure formation process, rather than to the cluster.

We note also that diffuse bridges of radio emission connecting the halo to the relic have been observed in a few clusters, including Coma (Kim et al. 1989; Giovannini et al. 1990) and A2255 (Govoni et al. 2005). In the Coma cluster Kronberg et al. (2007), confirmed by Brown and Rudnick (2011), detected a radio cloud surrounding both the halo and the relic source. The radio cloud size corresponds to about $4 \mathrm{Mpc}$ if it is associated with the Coma cluster, and is the most extensive complex of synchrotron emitting plasma yet seen in cosmic large-scale structure. In A2255, in addition to the radio bridge, low brightness emission located far away from the cluster center has been detected (Pizzo et al. 2008).

All the mentioned large-scale diffuse radio emissions might trace the process of a large-scale structure formation, where cosmic shocks that originated in complex merger events are able to amplify magnetic fields and accelerate synchrotron electrons along cluster filaments. In many cases the physical nature of these structures remains, however, ambiguous; candidates include cosmological filaments, unevolved 
protoclusters in the process of formation, and or large-scale debris near rich, merging clusters.

In any case, all these data support the existence of an intergalactic magnetic field more widespread and somewhat lower than that in the intracluster medium within clusters. This field may represent the seed field for galaxies and clusters, and may play an important role in the formation of large-scale structure. These are important results since they allow expanding our knowledge of magnetic fields from clusters to filaments, and are a fundamental step to understanding the origin and properties of cosmological scale magnetic fields. We are moving from magnetic field structures up to $1 \mathrm{Mpc}$ scale to structures 3-5 times more extended. Attempts to probe magnetic field strength on larger local-Universe scales have also been done via Faraday rotation studies (Xu et al. 2006).

The measurement of extremely weak magnetic fields in the voids and large-scale structure is a challenging task and up to now only upper bounds have been derived using various techniques. The tightest upper bounds come from the search for the Faraday rotation of polarization of the radio emission from distant quasars (e.g. Blasi et al. 1999), and from the effect of magnetic fields on the anisotropy of Cosmic Microwave Background radiation (e.g. Barrow et al. 1997; Durrer et al. 2000). A recent approach, using the cascade emission from blazars has been found to be promising (e.g. Neronov and Vovk 2010; Dolag et al. 2011; Tavecchio et al. 2011; Taylor et al. 2011). The multi-TeV gamma-ray flux from distant blazars is strongly attenuated by pair production of the infrared/optical extragalactic background light, initiating electromagnetic cascades in the intergalactic space, whose charged component is deflected by the extragalactic magnetic fields. Observable effects of these cascades include the delayed "echoes" of multi-TeV gamma-ray flares or gammaray bursts and the appearance of extended emission around point-like gamma-ray sources. From Fermi observations, lower bounds are at levels of $10^{-17}$ to $10^{-15} \mathrm{G}$, depending on the assumptions. Dolag et al. (2011) derive that these magnetic fields are likely to fill at least $60 \%$ of space, thus imposing constraints on the magnetic field origin: either the magnetic fields are produced by a volume filling process (e.g. primordially) or very efficient transport mechanisms must be present to distribute the locally generated magnetic fields into the space.

\section{Models of the origin of diffuse sources}

The connection between extended diffuse radio sources and cluster evolution, in particular the link between merger processes and halos/relics, on one hand, and the link between mini-halos and relaxed clusters, on the other hand, is well established as reported in this review. Major cluster mergers can supply energy to the radio emitting particles, as well as amplify magnetic fields (Röttiger et al. 1999a; Dolag et al. 1999, 2002; Ryu et al. 2008). It has been shown that the magnetic field strength can grow during the process of cluster formation, from initial fields of the order of $10^{-9} \mathrm{G}$ at $z=15$ to final fields of the order of $\mu \mathrm{G}$ at $z=0$. This magnetic field amplification is spread over the entire history of cluster formation, and related to the various merger processes, therefore it is not expected to directly affect the formation of diffuse radio sources. 
Since magnetic fields have been found to be ubiquitous in galaxy clusters (see Sect. 7.4), the crucial ingredient for the existence of diffuse synchrotron radio sources is the presence of relativistic particles. Radio halos are most difficult to explain, because of their very large size. Radiating electrons cannot travel such large distances within their lifetime, because of strong radiative losses by synchrotron and inverse Compton emission. Two main classes of models have been suggested for the origin of relativistic electrons present in the cluster volume and responsible for the diffuse radio emission: the primary electron model which predict that relativistic particles are continuously accelerated in the cluster volume, and the hadronic (secondary electrons) model, in which relativistic electrons are produced through the cluster volume by pp collisions.

We introduce here these models and summarize their expectations, to draw a picture of the formation scenario of diffuse radio sources. For a detailed analysis of the theoretical studies on this topic we refer to recent papers (e.g. Enßlin et al. 2011; Brunetti 2011 and references therein).

\subsection{Primary electrons}

Primary relativistic electrons are present in the cluster volume, because they were injected by AGN activity (quasars, radio galaxies, etc.), or by star formation in normal galaxies (supernovae, galactic winds, etc.) during the cluster dynamical history (see Blasi et al. 2007 for a review). This population of electrons suffers strong radiation losses mainly because of synchrotron and inverse Compton emission, thus reacceleration is needed to maintain their energy to the level necessary to produce the observed synchrotron radio emission in relatively weak magnetic fields (e.g. Jaffe 1977; Schlickeiser et al. 1987; Brunetti et al. 2001; Petrosian 2001; Brunetti and Lazarian 2011b). The possibility that electrons are accelerated from the sub-relativistic electrons has also been considered in the literature (e.g. Dogiel et al. 2007 and references therein).

For the above reasons, primary electron models can also be referred to as reacceleration models. There are two main ways that entail the transfer of energy from the cluster ICM to the radiating particles: these are cluster turbulence and cluster shocks.

\subsubsection{Reacceleration by turbulence: radio halos and mini-halos}

Simulations show that during cluster mergers, turbulence is generated throughout the cluster over $\sim$ Mpc scales. Energy can thus be transferred from the ICM into the non-thermal component through resonant or non-resonant interaction of electrons with MHD turbulence (see e.g. Brunetti et al. 2001, 2004; Petrosian 2001; Fujita et al. 2003; Cassano and Brunetti 2005; Xu et al. 2009, 2010). The emerging scenario is that turbulence reacceleration is likely the major mechanism responsible for the supply of energy to the electrons radiating in radio halos. Turbulent acceleration is like a second-order Fermi process, i.e. related with a random processes, and consequently not quite efficient. The time during which the process is effective is relatively short (a few $10^{8}$ years), so that the radio emission is expected to correlate with ongoing or most recent merger events (e.g. Brown et al. 2011; Feretti et al. 2004a; Enßlin et al. 2011). 
These models predict that reaccelerated electrons will have a maximum energy $\left(\gamma \sim 10^{5}\right)$ which produces a high frequency cutoff in the resulting synchrotron spectrum (Brunetti 2004). Thus a high frequency steepening of the integrated spectrum is expected, as well as a radial steepening and/or a complex spatial distribution of the spectral index between two frequencies, the latter due to different reacceleration processes in different cluster regions. The evidence of the presence of faint diffuse magnetic fields in all galaxy clusters (see Sect. 7.4), the behavior of radio spectra (see Sect. 4.1), the existence of ultra-steep radio halos (Sect. 4.1.3), the correlation between radio spectra and temperature (Sect. 4.1.2), the association between radio halos and cluster mergers (Sect. 4.3.2), and the fact that halos are not common in galaxy clusters (Kuo et al. 2004), are supportive of primary electron models for radio halos. The radio power-X-ray luminosity correlation (Sect. 4.3) is easily reproduced by reacceleration models (see e.g. Brunetti et al. 2009).

In mini-halos, the MHD turbulence associated with the cool-core region has been also suggested to be responsible for reacceleration of radiating particles (Gitti et al. 2002). This is consistent with the spectral properties and the observed correlation between the mini-halo radio power and the cooling rate power (Gitti et al. 2004). Another possibility, recently suggested by Mazzotta and Giacintucci (2008) and ZuHone et al. (2011), is that turbulence in cool-core clusters could arise from the sloshing motions of the cluster core gas. This hypothesis is supported by the detection of spiralshaped cold fronts in the X-ray emission of cool-core clusters, suggested to be the signature of gas sloshing (see e.g. Giacintucci et al. 2011b).

\subsubsection{Reacceleration by shocks: relics}

Shock acceleration is a first-order Fermi process of great importance in radio astronomy, as it is recognized as the mechanism responsible for particle acceleration in supernova remnants. The acceleration occurs diffusively, in that particles scatter back and forth across the shock, gaining at each crossing and recrossing an amount of energy proportional to the energy itself. The acceleration efficiency is mostly determined by the shock Mach number.

Current observations globally favor the scenario that cluster shocks are strictly linked to relics (Sarazin 1999; Keshet et al. 2004). The production of outgoing shock waves at the cluster periphery with Mach Number 2-3 is indeed observed in numerical simulations of cluster merger events and more generally in the large-scale structure formation (Miniati et al. 2000; Ryu et al. 2003, 2008; Vazza et al. 2009). Accelerated electrons have been suggested either to be thermal ICM electrons (Enßlin et al. 1998), or relativistic electrons released by a former active radio galaxy (Enßlin 2001; Enßlin and Brüggen 2002; Hoeft et al. 2004).

Because of the short electron radiative lifetimes, radio emission is produced close to the location of the shock waves. These models also predict that the magnetic field within the relic is aligned with the shock front, and that the radio spectrum is flatter at the shock edge, where the radio brightness is expected to decline sharply. These expectations are consistent with the classic elongated structure of relics, almost perpendicular to the merger axis, and their polarization properties. Vazza et al. (2012) show that the detection frequency of radio relics and the fact that they are also detected in cluster peripheries is consistent with their link to shocks. 
The detection of shocks in the cluster outskirts is difficult with current instruments, because of the very low gas density and thus X-ray brightness in these regions (for a recent review see Markevitch and Vikhlinin 2007). In the Coma cluster, Feretti and Neumann (2006), analyzing Newton-XMM data, did not detect the temperature jump implied by the presence of a shock at the relic location, although the brightness trend may be consistent with the shock. Solovyeva et al. (2008) presented evidence of a shock at the location of the relics in A548b. A weak shock has been detected by Macario et al. (2011) in A754. Recently, shocks have been detected with Suzaku in A3376 (Akamatsu et al. 2011a) and A3667 (Akamatsu et al. 2011b).

It cannot be excluded that shock acceleration may also be efficient in some particular regions of a halo (e.g. Markevitch 2010), however, the radio emission of halos can be very extended up to large scales, thus it is hardly associated with localized shocks. Moreover, some clusters exhibit a spatial correlation between the radio halo emission and the hot gas regions (Govoni et al. 2004), however, this is not a general feature, and in some cases the hottest gas regions do not exhibit radio emission.

\subsection{Secondary electrons}

In the hadronic model, secondary electrons are injected as secondary particles by inelastic nuclear collisions between the relativistic protons and the nuclei of the thermal ambient intracluster medium (e.g. Dennison 1980; Blasi and Colafrancesco 1999; Dolag and Enßlin 2000; Keshet and Loeb 2010). The protons diffuse on large scale because their energy losses are negligible. They can continuously produce in situ electrons, distributed through the cluster volume. The possibility that the high-energy electrons, responsible for the synchrotron emission, arise from the decay of secondary products of the neutralino annihilation in the dark matter halos of galaxy clusters has also been suggested (Colafrancesco and Mele 2001).

Secondary electron models have been proposed for the emission of radio halos (see e.g. Dennison 1980; Dolag and Enßlin 2000) and of mini-halos (Pfrommer and Enßlin 2004). They cannot work for relics, since peripheral cluster regions do not host a sufficiently dense thermal proton population required as targets for the efficient production of secondary electrons.

Secondary electron models can reproduce the basic properties of the radio halos provided that the strength of the magnetic field averaged over the emitting volume is larger than a few $\mu \mathrm{G}$. In this case, they predict synchrotron power-law spectra, which are independent of cluster location, i.e. do not show any features and/or radial steepening, and the spectral index values are flatter than $\alpha \sim 1.5$ (e.g. Brunetti 2004). The profiles of the radio emission should be steeper than those of the X-ray gas (e.g. Govoni et al. 2001b). Since the radio emitting electrons originate from protons accumulated during the cluster formation history, no correlation to recent mergers is expected, but halos should be present in virtually all clusters. Moreover, emission of gamma rays and of neutrinos is predicted.

Recently, a universal linear correlation between radio and X-ray surface brightness in halos and mini-halos was found (Keshet and Loeb 2010), assuming secondary electron models and a relatively strong magnetized IGM $(>3 \mu \mathrm{G})$. This model reproduces the observed radio spectral steepening, but it requires a very high central magnetic field $(>10 \mu \mathrm{G})$. 


\subsection{Hybrid models}

The intracluster medium is a complex mixture of thermal and non-thermal protons and electrons. It has been suggested in that halos could arise from both primary and secondary electrons. Relativistic protons are an important component of relativistic ICM particles, and they are an important origin of relativistic electrons. MHD turbulence can reaccelerate both primary and secondary particles, producing radio emission in agreement with observational results (see e.g. Miniati et al. 2001). The relative contribution of these two components is constrained by the observational properties. Constraints on the energy budget of the various components have been derived by Brunetti and Blasi (2005) and more recently by Brunetti and Lazarian (2011a). These authors discuss also that secondary electrons could give rise to $\mathrm{Mpc}$-scale halos characterized by very low surface brightness and radio power, not yet observed because of observational limits of present radio telescopes, but which should be detected by the incoming new radio telescopes.

\subsection{Constraints from gamma-ray emission}

The collisions producing secondary electrons also lead to gamma-ray emission through the production and subsequent decays of neutral pions. The implications of gamma-ray detections from galaxy clusters are largely discussed in literature (see e.g. Blasi et al. (2007) and references therein). However, diffuse gammaray emission has not yet been detected from galaxy clusters (Reimer et al. 2003; Ackermann et al. 2010). These non-detections constrain the possible energy density in cosmic-ray protons and, therefore, the density of relativistic electrons that may be produced from these.

The Fermi-LAT Gamma-ray Space Telescope greatly improved the sensitivity of observations at $\mathrm{MeV} / \mathrm{Gev}$ energies and reported gamma-ray upper limits for a large sample of clusters (Ackermann et al. 2010). Jeltema and Profumo (2011) examined the secondary electron models for the origin of halos in the light of these new Fermi results. The non-detection of gamma-ray emission from clusters puts constraints to the possible density of secondary relativistic electrons and imply a minimum magnetic field strength to reproduce the observed emission from radio halos. For clusters such as A1914 and A2256, average magnetic fields at least of the order of several $\mu$ Gauss are needed to avoid an overproduction of gamma rays, not seen by Fermi (2011). The implied magnetic fields in hadronic models are in excess of, or close to, the largest cluster magnetic field values obtained with Faraday Rotation Measures (see Sect. 7). This seems problematic for the hadronic origin of radio halos. However, at present, due to the uncertainties, secondary electron models are not ruled out by Fermi gamma-ray data even if required magnetic fields are in some clusters higher than values obtained with other observations. This result shows the importance of high-energy data in understanding non-thermal emission in galaxy clusters.

\section{Future}

Our knowledge of non-thermal phenomena in galaxy clusters has significantly improved in the past years, owing to good and deep observations and advanced model- 
ing. Nevertheless, new observations will be necessary to solve the outstanding issues faced in determining what is the dominating mechanism in the formation and maintenance of the diffuse radio emission, in particular: (1) search for new sources of the different classes, in particular at low powers and at large redshifts, to improve the information on the statistical properties of cluster diffuse sources; (2) obtain polarization information on halos, and perform Rotation Measure studies of embedded and background radio sources, to get a detailed knowledge of the magnetic field strength, structure, and degree of ordering; (3) derive accurate integrated spectra on a large frequency range and detailed spectral index distributions at high resolution.

New generation instruments, which are becoming available, as the EVLA and LOFAR, or are planned, as the SKA precursors (ASKAP, MeerKAT), and SKA, will allow a breakthrough in these studies.

Owing to the new capabilities of the EVLA, in particular sensitivity, frequency agility, and spectral capability, it will be possible to detect faint radio emission and trace the magnetic fields in galaxy clusters.

The new radio telescope LOFAR will explore the Universe at low frequencies, as shown e.g. by the first results on the cluster A2256 (van Weeren et al. 2012). As diffuse cluster radio sources are bright at low frequency, LOFAR will be able to detect thousands of such sources up to the epoch of cluster formation, in particular halos and relics with ultra-steep spectra, which are missed at $\mathrm{cm}$ frequencies. This will allow the investigation of the occurrence of diffuse cluster radio sources. Their properties will be derived as a function of cluster properties up to the epoch at which the first massive clusters assembled $(z \sim 1)$, testing predictions that cluster merging is rampant at high redshift. The detection of synchrotron radiation at the lowest possible levels will allow the measurement of magnetic fields in clusters and in even more rarefied regions of the intergalactic space, and the investigation of the relation between the formation of magnetic fields and the formation of the large-scale structure in the universe. With LOFAR, owing to the long wavelengths involved, it will also be possible to obtain Faraday rotation down to $0.1-1 \mathrm{rad} \mathrm{m}^{2}$, which are very difficult to measure with the current instruments at $\mathrm{cm}$ wavelengths. The detection of very low Rotation Measures implies in turn the detection of very faint magnetic fields therefore LOFAR will become the radio telescope to measure the weakest cosmic magnetic fields so far.

Two projects planned with the Australian Square Kilometre Array Pathfinder (ASKAP), i.e. the deep radio sky survey project EMU (Evolutionary Map of the Universe) and the polarization survey POSSUM (Polarisation Sky Survey of the Universe's Magnetism) will allow the detection of diffuse radio sources and the improvement of Faraday rotation information in clusters and in the overall intergalactic medium. The first science with the MeerKAT array in South Africa region, in particular the MIGHTEE (MeerKAT International GigaHertz Tiered Extragalactic Exploration Survey) project will cover a wide range of astrophysics, including non-thermal phenomena in clusters.

APERTIF phased arrays that are being installed in WSRT will dramatically increase the survey speed for WSRT. This will enable the WODAN (Westerbork Observations of the Deep APERTIF Northern-Sky) project, a survey complementary to EMU. 
The most innovative studies will be performed with SKA: they are summarized in "Science with the Square Kilometre Array" (Carilli and Rawlings 2004; for cluster and intergalactic medium studies see e.g. Feretti et al. 2004c; Feretti and JohnstonHollitt 2004; Keshet et al. 2004; Gaensler et al. 2004; Beck 2011). Recently, Krause et al. (2009) investigate the potential of the Square Kilometer Array (SKA) for measuring the magnetic fields in clusters of galaxies via Faraday rotation of background polarized sources. They derive the distribution of RM due to clusters, by using an average gas density distribution and adopting models for the population of cluster radio sources, for the cluster mass distribution, and for the source polarization. They calculate the statistics for background RM measurements in clusters at different redshift and different SKA exposures, arrays and completion levels in the mid frequency range. They conclude that the full SKA will be able to make very detailed magnetic field structure measurements of clusters with more than 100 background sources in clusters up to $z=0.5$, and more than 1000 background radio sources in nearby clusters. Bogdanovic et al. (2011) analyze different physical mechanisms operating in the ICM, i.e. conduction-driven MHD instabilities and turbulence-dominated instabilities, and derive that these deeply affect the structure of the cluster magnetic field producing different field topologies. They evaluate the effects of the two mechanisms on the Faraday RM of background radio sources and conclude that future spectropolarimetrimetric measurements with EVLA and SKA will have sufficient sensitivity to discriminate between them.

Observations in the radio domain will need to be complemented by data at all other wavelengths, in particular optical, X-ray and gamma-ray data, to establish the cluster conditions, the merger evolutionary stage, the presence and properties of shocks, the signatures of cluster turbulence, and to derive firm correlations on large samples and over a large range of parameters between radio properties and cluster parameters. Finally, the study of the non-thermal inverse Compton emission in the hard X-ray and extreme ultraviolet domains needs to be pursued on several clusters, with and without diffuse emission, to get independent information on the existence of relativistic particles.

Acknowledgements We are grateful to W.D. Cotton for a careful and critical reading of the manuscript. We thank R. Morganti and T. Courvoisier for helpful suggestions.

Open Access This article is distributed under the terms of the Creative Commons Attribution License which permits any use, distribution, and reproduction in any medium, provided the original author(s) and the source are credited.

\section{References}

Ackermann M, Ajello M, Allafort A et al (2010) Astrophys J 717:L71

Akamatsu H, Takizawa M, Nakazawa K et al (2011a) Publ Astron Soc Jpn (in press). arXiv:1112.5955

Akamatsu H, de Plaa J, Kaastra J et al (2011b) Publ Astron Soc Jpn (in press). arXiv:1111.5162

Bacchi M, Feretti L, Giovannini G, Govoni F (2003) Astron Astrophys 400:465

Bagchi J, Enßlin TA, Miniati F et al (2002) New Astron 7:249

Bagchi J, Durret F, Neto GBL, Paul S (2006) Science 314:791

Bagchi J, Jacob J, Gopal-Krishna et al (2009) Mon Not R Astron Soc 399:601

Bagchi J, Sirothia SK, Werner N et al (2011) Astrophys J 736:L8 
Ballarati B, Feretti L, Ficarra A et al (1981) Astron Astrophys 100:323

Barrena R, Girardi M, Boschin W, Dasi M (2009) Astron Astrophys 503:357

Barrow JD, Ferreira PG, Silk J (1997) Phys Rev Lett 78:3610

Beck R (2011) In: Soida M, Otmianowska-Mazur K, de Gouveia EM, Lazarian A (Eds) Proc of magnetic fields in the universe: from laboratory and stars to primordial structures (in press). arXiv:1111.5802

Blanton EL, Randall SW, Clarke TE et al (2011) Astrophys J 737:99

Blasi P, Colafrancesco S (1999) Astropart Phys 12:169

Blasi P, Burles S, Olinto AV (1999) Astrophys J 514:L79

Blasi P, Gabici S, Brunetti G (2007) Int J Mod Phys A 22:681

Bogdanovic T, Reynolds CS, Massey R (2011) Astrophys J 731:7

Böhringer H, Schuecker P (2002) Astrophys space sci libr, vol 272. Kluwer Academic, Dordrecht, p 133

Böhringer H, Werner N (2010) Astron Astrophys Rev 18:127

Bonafede A, Giovannini G, Feretti L et al (2009a) Astron Astrophys 494:429

Bonafede A, Feretti L, Giovannini G, Govoni F et al (2009b) Astron Astrophys 503:707

Bonafede A, Feretti L, Murgia M et al (2010) Astron Astrophys 513:A30

Bonafede A, Govoni F, Feretti L et al (2011) Astron Astrophys 530:A24

Boschin W, Girardi M, Barrena R, Biviano A et al (2004) Astron Astrophys 416:839

Boschin W, Girardi M, Spolaor M, Barrena R (2006) Astron Astrophys 449:461

Boschin W, Barrena R, Girardi M (2010) Astron Astrophys 521:A78

Brentjens MA (2008) Astron Astrophys 489:69

Brentjens MA, de Bruyn G (2005) Astron Astrophys 441:1217

Brown S, Rudnick L (2011) Mon Not R Astron Soc 412:2

Brown S, Duesterhoeft J, Rudnick L (2011) Astrophys J 727:25

Brüggen M, Ruszkowski M, Simionescu A et al (2005) Astrophys J 631:L21

Brüggen M, van Weeren RJ, Röttgering HJA (2011) Mem Soc Astron Ital 82:627

Brunetti G (2004) J Korean Astron Soc 37:493

Brunetti G (2011) Mem Soc Astron Ital 82:515

Brunetti G, Blasi P (2005) Mon Not R Astron Soc 363:1173

Brunetti G, Lazarian A (2011a) Mon Not R Astron Soc 410:127

Brunetti G, Lazarian A (2011b) Mon Not R Astron Soc 412:817

Brunetti G, Setti G, Feretti L, Giovannini G (2001) Mon Not R Astron Soc 320:365

Brunetti G, Blasi P, Cassano R, Gabici S (2004) Mon Not R Astron Soc 350:1174

Brunetti G, Venturi T, Dallacasa D, Cassano R et al (2007) Astrophys J 670:L5

Brunetti G, Cassano R, Dolag K, Setti G (2009) Astron Astrophys 507:661

Buote DA (2001) Astrophys J 553:L15

Burn BJ (1966) Mon Not R Astron Soc 133:67

Burns JO, Sulkanen ME, Gisler GR, Perley RA (1992) Astrophys J 388:L49

Carilli C, Rawlings S (eds) (2004) Science with the square kilometre array. New astronomy reviews, vol 48. Elsevier, Amsterdam

Carilli CL, Taylor GB (2002) Annu Rev Astron Astrophys 40:319

Cassano R (2010) Astron Astrophys 517:10

Cassano R, Brunetti G (2005) Mon Not R Astron Soc 357:1313

Cassano R, Brunetti G, Setti G (2006) Mon Not R Astron Soc 369:1577

Cassano R, Brunetti G, Setti G (2007) Mon Not R Astron Soc 378:156

Cassano R, Gitti M, Brunetti G (2008) Astron Astrophys 486:L31

Cassano R, Ettori S, Giacintucci S et al (2010) Astrophys J 721:82

Cassano R, Brunetti G, Venturi T (2011) J Astrophys Astron, 32:519

Clarke TE, Enßlin TA (2006) Astron J 131:2900

Clarke TE, Kronberg PP, Böhringer H (2001) Astrophys J 547:L111

Cohen AS, Clarke TE (2011) Astron J 141:149

Colafrancesco S, Mele B (2001) Astrophys J 562:24

Condon JJ, Cotton WD, Greisen EW et al (1998) Astron J 115:1693

Dallacasa D, Brunetti G, Giacintucci S et al (2009) Astrophys J 699:1288

De Grandi S, Molendi S (2001) Astrophys J 551:153

Dennison B (1980) Astrophys J 239:L93

Dogiel VA, Colafrancesco S, Ko CM et al (2007) Astron Astrophys 461:433

Dolag K, Enßlin TA (2000) Astron Astrophys 362:151

Dolag K, Bartelmann M, Lesch H (1999) Astron Astrophys 348:351 
Dolag K, Schindler S, Govoni F, Feretti L (2001) Astron Astrophys 378:777

Dolag K, Bartelmann M, Lesch H (2002) Astron Astrophys 387:383

Dolag K, Kachelriess M, Ostapchenko S, Tomàs R (2011) Astrophys J 727:L4

Durrer R, Ferreira PG, Kahniashvili T (2000) Phys Rev D 61:043001

Eckert D (2011) Mem Soc Astron Ital 82:527

Eilek JA, Owen FN (2002) Astrophys J 567:202

Enßlin TA, Brüggen M (2002) Mon Not R Astron Soc 331:1011

Enßlin TA, Gopal-Krishna (2001) Astron Astrophys 366:26

Enßlin TA, Vogt C (2003) Astron Astrophys 401:835

Enßlin TA, Torsten A, Biermann PL et al (1998) Astron Astrophys 332:395

Enßlin TA, Pfrommer C, Miniati F, Subramanian K (2011) Astron Astrophys 527:A99

Fabian AC (1994) Annu Rev Astron Astrophys 32:277

Fabian AC, Sanders JS, Allen SW et al (2003) Mon Not R Astron Soc 344:L43

Felten JE (1996) Clust Lensing Future Universe 88:271

Feretti L (1999) In: Böhringer H, Feretti L, Schuecker P (eds) Proceedings of the Ringberg Workshop, April 19-23, p 3

Feretti L, (2002) In: Universe Low Radio Freq. IAU, vol 199, p 133

Feretti L (2003) In: Matter and energy in clusters of galaxies. Astronomical society of the pacific conference series, vol 301, p 143

Feretti L, Giovannini G (1996) In: Ekers RD, Fanti C, Padrielli L (eds) 175th IAU sympos, p 333

Feretti L, Giovannini G (2008) In: Plionis M, L'opez-Cruz O, Hughes D (eds) Lect notes phys, vol 740. Springer, Dordrecht, p 143

Feretti L, Johnston-Hollitt M (2004) New Astron Rev 48:1145

Feretti L, Neumann DM (2006) Astron Astrophys 450:21

Feretti L, Dallacasa D, Giovannini G, Tagliani A (1995) Astron Astrophys 302:680

Feretti L, Böhringer H, Giovannini G, Neumann D (1997a) Astron Astrophys 317:432

Feretti L, Giovannini G, Böhringer H (1997b) New Astron 2:501

Feretti L, Dallacasa D, Govoni F et al (1999a) Astron Astrophys 344:472

Feretti L, Perley R, Giovannini G, Andernach H (1999b) Astron Astrophys 341:29

Feretti L, Fusco-Femiano R, Giovannini G, Govoni F (2001) Astron Astrophys 373:106

Feretti L, Brunetti G, Giovannini G, Kassim N, Orrù E, Setti G (2004a) J Korean Astron Soc 37:315

Feretti L, Orrù E, Brunetti G, Giovannini G, Kassim N, Setti G (2004b) Astron Astrophys 423:111

Feretti L, Burigana C, Enßlin TA (2004c) New Astron Rev 48:1137

Feretti L, Schuecker P, Böhringer H et al (2005) Astron Astrophys 444:157

Feretti L, Bacchi M, Slee OB et al (2006) Mon Not R Astron Soc 368:544

Feretti L, Bonafede A, Giovannini G, Govoni F, Murgia M (2010) Highlights Astron 15:459

Ferrari C, Maurogordato S, Cappi A, Benoist C (2003) Astron Astrophys 399:813

Ferrari C, Govoni F, Schindler S et al (2008) Space Sci Rev 134:93

Ferrari C, Intema HT, Orrù E et al (2011) Astron Astrophys 534:L12

Finoguenov A, Sarazin CL, Nakazawa K, Wik DR et al (2010) Astrophys J 715:1143

Fujita Y, Takizawa M, Sarazin CL (2003) Astrophys J 584:190

Fujita Y, Kohri K, Yamazaki R, Kino M (2007) Astrophys J 663:L61

Fusco-Femiano R, dal Fiume D, Feretti L et al (1999) Astrophys J 513:L21

Fusco-Femiano R, Orlandini M, Brunetti G et al (2004) Astrophys J 602L:73

Fusco-Femiano R, Landi R, Orlandini M (2005) Astrophys J 624:L69

Fusco-Femiano R, Landi R, Orlandini M (2007) Astrophys J 654:L9

Fusco-Femiano R, Orlandini M, Bonamente M, Lapi A (2011) Astrophys J 732:85

Gabici S, Blasi P (2003) Astrophys J 583:695

Gaensler BM, Beck R, Feretti L (2004) New Astron Rev 48:1003

Garrington ST, Conway RG (1991) Mon Not R Astron Soc 250:198

Garrington ST, Leahy JP, Conway RG, Laing RA (1988) Nature 331:147

Gavazzi G, Trinchieri G (1983) Astrophys J 270:410

Giacintucci S (2011) Mem Soc Astron Ital 82:541

Giacintucci S, Venturi T, Brunetti G, Bardelli S et al (2005) Astron Astrophys 440:867

Giacintucci S, Venturi T, Macario G, Dallacasa D et al (2008) Astron Astrophys 486:347

Giacintucci S, Venturi T, Brunetti G, Dallacasa D et al (2009a) Astron Astrophys 505:45

Giacintucci S, Venturi T, Cassano R, Dallacasa D, Brunetti G (2009b) Astrophys J 704:54

Giacintucci S, Dallacasa D, Venturi T, Brunetti G et al (2011a) Astron Astrophys 534:57 
Giacintucci S, Markevitch M, Brunetti G et al (2011b) Astron Astrophys 525:L10

Giovannini G, Feretti L (2000) New Astron 5:335

Giovannini G, Feretti L (2002) In: Astrophys space sci libr, vol 272. Kluwer Academic, Dordrecht, p 197

Giovannini G, Feretti L (2004) J Korean Astron Soc 37:323

Giovannini G, Kim KT, Kronberg PP, Venturi T (1990) In: Beck R, Kronberg PP, Wielebinski R (eds) IAUS, vol 140, p 492

Giovannini G, Feretti L, Stanghellini C (1991) Astron Astrophys 252:528

Giovannini G, Feretti L, Venturi T, Kim K-T, Kronberg PP (1993) Astrophys J 406:399

Giovannini G, Tordi M, Feretti, L (1999) New Astron 4:141

Giovannini G, Bonafede A, Feretti L et al (2009) Astron Astrophys 507:1257

Giovannini G, Bonafede A, Feretti L et al (2010) Astron Astrophys 511:L5

Giovannini G, Feretti L, Girardi M et al (2011) Astron Astrophys 530:5

Girardi M, Biviano A (2002) In: Astrophys space sci libr, vol 272. Kluwer Academic, Dordrecht, p 39

Gitti M, Brunetti G, Setti G (2002) Astron Astrophys 386:456

Gitti M, Brunetti G, Feretti L, Setti G (2004) Astron Astrophys 417:1

Gitti M, Feretti L, Schindler S (2006) Astron Astrophys 448:853

Gitti M, Ferrari C, Domainko W et al (2007) Astron Astrophys 470:L25

Govoni F, Feretti L (2004) Int J Mod Phys D 13(8):1549

Govoni F, Taylor GB, Dallacasa D et al (2001a) Astron Astrophys 379:807

Govoni F, Enßlin TA, Feretti L, Giovannini G (2001b) Astron Astrophys 369:441

Govoni F, Feretti L, Giovannini G et al (2001c) Astron Astrophys 376:803

Govoni F, Markevitch M, Vikhlinin A et al (2004) Astrophys J 605:695

Govoni F, Murgia M, Feretti L et al (2005) Astron Astrophys 430:L5

Govoni F, Murgia M, Feretti L, et al (2006) Astron Astrophys 460:425

Govoni F, Murgia M, Markevitch M et al (2009) Astron Astrophys 499:371

Govoni F, Dolag K, Murgia M et al (2010) Astron Astrophys 522:A105

Govoni F, Murgia M, Giovannini G, Vacca V, Bonafede A (2011) Astron Astrophys 529:69

Guidetti D, Murgia M, Govoni F et al (2008) Astron Astrophys 483:699

Guidetti D, Laing RA, Murgia M et al (2010) Astron Astrophys 514:A50

Guidetti D, Laing RA, Bridle AH et al (2011) Mon Not R Astron Soc 413:2525

Hanisch RJ (1982) Astron Astrophys 116:137

Harris DE, Stern CP, Willis AG, Dewdney PE (1993) Astron J 105:769

Hoeft M, Brüggen M, Yepes G (2004) Mon Not R Astron Soc 347:389

Jaffe WJ (1977) Astrophys J 212:1

Jaffe WJ, Rudnick L (1979) Astrophys J 233:453

Jeltema TE, Profumo S (2011) Astrophys J 728:53

Johnston-Hollitt M (2003) PhD Thesis, University of Adelaide

Johnston-Hollitt M, Ekers RD, Hunstead RW, Clay RW, Wieringa MH (2002) Highlights Astron 12:535

Johnston-Hollitt M, Hollitt CP, Ekers RD (2004) In: Uyaniker B, Reich W, Wielebinski R (eds) The magnetized interstellar medium, p 13

Kale R, Dwarakanath KS (2010) Astrophys J 718:939

Kempner JC, David LP (2004) Mon Not R Astron Soc 349:385

Kempner JC, Sarazin CL (2001) Astrophys J 548:639

Kempner JC, Blanton EL, Clarke TE, Enßlin TA et al (2004) In: Reiprich T, Kempner J, Soker $\mathrm{N}$ (eds) Proceedings of the riddle of cooling flows in galaxies and clusters of galaxies. http://www.astro.virginia.edu/coolflow

Keshet U, Loeb A (2010) Astrophys J 722:737

Keshet U, Waxman E, Loeb A, Springel V, Hernquist L (2003) Astrophys J 585:128

Keshet U, Waxman E, Loeb A (2004) Astrophys J 617:281

Kim K-T, Kronberg PP, Giovannini G, Venturi T (1989) Nature 341:720

Kim K-T, Kronberg PP, Dewdney PE, Landecker TL (1990) Astrophys J 355:29

Kim K-T, Tribble PC, Kronberg PP (1991) Astrophys J 379:80

Komissarov SS, Gubanov AG (1994) Astron Astrophys 285:27

Krause M, Alexander P, Bolton R, Geisbüsch J, Green DA, Riley J (2009) Mon Not R Astron Soc 400:646

Kronberg PP (1994) Rep Prog Phys 57:325

Kronberg PP, Kothes R, Salter CJ, Perillat P (2007) Astrophys J 659:267

Kuchar P, Enßlin TA (2011) Astron Astrophys 529:A13

Kunz MW, Schekochihin AA, Cowley SC et al (2011) Mon Not R Astron Soc 410:2446 
Kuo P-H, Hwang C-Y, Ip W-H (2004) Astrophys J 604:108

Laing RA (1988) Nature 331:149

Laing RA, Bridle AH, Parma P, Murgia M (2008) Mon Not R Astron Soc 391:521

Large MI, Mathewson DS, Haslam CGT (1959) Nature 183:1663

Lawler JM, Dennison B (1982) Astrophys J 252:81

Liang H, Hunstead RW, Birkinshaw M, Andreani P (2000) Astrophys J 544:686

Macario G (2011) PhD Thesis Astronomy Dept, Bologna University

Macario G, Venturi T, Brunetti G et al (2010) Astron Astrophys 517:43

Macario G, Markevitch M, Giacintucci S et al (2011) Astrophys J 728:82

Mao MY, Sharp R, Saikia DJ et al (2010) Mon Not R Astron Soc 406:2578

Markevitch M (2010) In: 12th Marcel Grossman Meeting, Paris, arXiv:1010.3660

Markevitch M, Vikhlinin A (2007) Phys Rep 443:1

Mason BS, Dicker SR, Korngut PM et al (2010) Astrophys J 716:739

Mathews WG, Brighenti F (2008) Astrophys J 676:880

Mazzotta P, Giacintucci S (2008) Astrophys J 675:L9

McNamara BR, Nulsen PEJ (2007) Annu Rev Astron Astrophys 45:117

McNamara BR, Rafferty DA, Birzan L et al (2006) Astrophys J 648:164

Middelberg E, Norris RP, Cornwell TJ, Voronkov MA et al (2008) Astron J 135:1276

Miley GK, Perola GG (1975) Astron Astrophys 45:223

Miniati F (2003) Mon Not R Astron Soc 342:1009

Miniati F, Ryu D, Kang H, Jones TW et al (2000) Astrophys J 542:608

Miniati F, Jones TW, Kang H, Ryu D (2001) Astrophys J 562:233

Murgia M, Govoni F, Feretti L et al (2004) Astron Astrophys 424:429

Murgia M, Govoni F, Markevitch M et al (2009) Astron Astrophys 499:679

Murgia M, Govoni F, Feretti L, Giovannini G (2010a) Astron Astrophys 509:86

Murgia M, Eckert D, Govoni F et al (2010b) Astron Astrophys 514:A76

Murgia M, Parma P, Mack K-H et al (2011) Astron Astrophys 526:A148

Neronov A, Vovk I (2010) Science 328:73

Noordam JE, de Bruyn AG (1982) Nature 299:597

Nuza SE, Hoeft M, van Weeren RJ, Gottlöber S, Yepes G (2012) Mon Not R Astron Soc 420:2006

Orrù E, Murgia M, Feretti L et al (2007) Astron Astrophys 467:943

Pacholczyk AG (1970) Radio astrophysics. Freeman, San Francisco

Pedlar A, Ghataure HS, Davies RD et al (1990) Mon Not R Astron Soc 246:477

Perley RA, Taylor GB (1991) Astron J 101:1623

Peterson JR, Fabian AC (2006) Phys Rep 427:1

Peterson JR, Paerels FBS, Kaastra JS et al (2001) Astron Astrophys 365:L104

Petrosian V (2001) Astrophys J 557:560

Pfrommer C, Enßlin TA (2004) Astron Astrophys 413:17

Pizzo RF, de Bruyn AG (2009) Astron Astrophys 507:639

Pizzo RF, de Bruyn AG, Feretti L, Govoni F (2008) Astron Astrophys 481:L91

Pizzo RF, de Bruyn AG, Bernardi G, Brentjens MA (2011) Astron Astrophys 525:A104

Randall SW, Clarke TE, Nulsen PEJ et al (2010) Astrophys J 722:825

Reid AD, Hunstead RW, Lemonon L, Pierre MM (1999) Mon Not R Astron Soc 302:571

Reimer O, Pohl M, Sreekumar P, Mattox JR (2003) Astrophys J 588:155

Reiprich TH, Böhringer H (2002) Astrophys J 567:716

Rengelink RB, Tang Y, de Bruyn AG et al (1997) Astron Astrophys Suppl Ser 124:259

Rephaeli Y, Gruber D (2002) Astrophys J 579:587

Rossetti M, Eckert D, Cavalleri BM et al (2011) Astron Astrophys 532:123

Röttgering HJA, Wieringa MH, Hunstead RW, Ekers RD (1997) Mon Not R Astron Soc 290:577

Röttiger K, Stone JM, Burns JO (1999a) Astrophys J 518:594

Röttiger K, Burns JO, Stone JM (1999b) Astrophys J 518:603

Rudnick L, Blundell KM (2003) Astrophys J 588:143

Rudnick L, Lemmerman JA (2009) Astrophys J 697:1341

Russel HR, van Weeren RJ, Edge AC et al (2011) Mon Not R Astron Soc 417:L1

Ryu D, Kang H, Hallman E, Jones TW (2003) Astrophys J 593:599

Ryu D, Kang H, Cho J, Das S (2008) Science 320:909

Sarazin CL (1999) Astrophys J 520:529

Sarazin CL (2002) In: Astrophys space sci libr, vol 272. Kluwer Academic, Dordrecht, p 1 
Schlickeiser R, Sievers A, Thiemann H (1987) Astron Astrophys 182:21

Schuecker P, Böhringer H, Reiprich TH, Feretti L (2001) Astron Astrophys 378:408

Sijbring LG (1993) PhD Thesis, University of Groningen

Slee OB, Roy AL, Murgia M, Andernach H, Ehle M (2001) Astron J 122:1172

Solovyeva L, Anokhin S, Feretti L et al (2008) Astron Astrophys 484:621

Subrahmanyan R, Beasley AJ, Goss WM, Golap K, Hunstead RW (2003) Astron J 125:1095

Tavecchio F, Ghisellini G, Bonnoli G, Foschini L (2011) Mon Not R Astron Soc 414:3566

Taylor GB, Perley RA (1993) Astrophys J 416:554

Taylor GB, Govoni F, Allen S, Fabian AC (2001) Mon Not R Astron Soc 326:2

Taylor GB, Fabian AC, Gentile G, et al (2007) Mon Not R Astron Soc 382:67

Taylor AM, Vovk I, Neronov A (2011) Astron Astrophys 529:144

Thierbach M, Klein U, Wielebinski R (2003) Astron Astrophys 397:53

Tribble PC (1991a) Mon Not R Astron Soc 253:147

Tribble PC (1991b) Mon Not R Astron Soc 250:726

Tucker W (1975) Radiation processes in astrophysics. MIT Press, Cambridge

Vacca V, Murgia M, Govoni F et al (2010) Astron Astrophys 514:71

Vacca V, Govoni F, Murgia M, Giovannini G, Feretti L et al (2011) Astron Astrophys 535:A82

Vallée JP, MacLeod MJ, Broten NW (1986) Astron Astrophys 156:386

Valtchanov I, Murphy T, Pierre M, Hunstead R, Lémonon L (2002) Astron Astrophys 392:795

van Weeren RJ, Röttgering HJA, Brüggen M, Cohen A (2009a) Astron Astrophys 505:991

van Weeren RJ, Röttgering HJA, Brüggen M, Cohen A (2009b) Astron Astrophys 508:75

van Weeren RJ, Röttgering HJA, Bagchi J et al (2009c) Astron Astrophys 506:1083

van Weeren RJ, Röttgering HJA, Brüggen M, Hoeft M (2010) Science 330:347

van Weeren RJ, Brüggen M, Röttgering HJA et al (2011a) Astron Astrophys 533:35

van Weeren RJ, Röttgering HJA, Brüggen M (2011b) Astron Astrophys 527:114

van Weeren RJ, Hoeft M, Röttgering HJA et al (2011c) Astron Astrophys 528:38

van Weeren RJ, Brüggen M, Röttgering HJA, Hoeft M (2011d) Mon Not R Astron Soc 418:230

van Weeren RJ, Röttgering HJA, Rafferty DA et al (2012) Astron Astrophys (submitted)

Vazza F, Brunetti G, Gheller C (2009) Mon Not R Astron Soc 395:1333

Vazza F, Brüggen M, van Weeren R, Bonafede A et al (2012) Mon Not R Astron Soc 421:1868

Venturi T (2011) Mem Soc Astron Ital 82:499

Venturi T, Bardelli S, Dallacasa D et al (2003) Astron Astrophys 402:913

Venturi T, Giacintucci S, Brunetti G et al (2007) Astron Astrophys 463:937

Venturi T, Giacintucci S, Dallacasa D et al (2008) Astron Astrophys 484:327

Venturi T, Giacintucci S, Cassano R et al (2009) In: The low frequency radio universe. ASP conf ser, vol 407, p 232

Vogt C, Enßlin TA (2003) Astron Astrophys 412:373

Vogt C, Enßlin TA (2005) Astron Astrophys 434:67

Wik DR, Sarazin CL, Finoguenov A et al (2009) Astrophys J 696:1700

Wik DR, Sarazin CL, Finoguenov A et al (2011) Astrophys J 727:119

Willson MAG (1970) Mon Not R Astron Soc 151:1

Xu Y, Kronberg PP, Habib S, Dufton QW (2006) Astrophys J 637:19

Xu H, Li H, Collins DC, Li S, Norman ML (2009) Astrophys J 698:L14

Xu H, Li H, Collins DC, Li S, Norman ML (2010) Astrophys J 725:2152

ZuHone J, Markevitch M, Brunetti G (2011) Mem Soc Astron Ital 82:632 